Research article urn:1sid:zoobank.org:pub:99B180D2-CCD2-4171-B640-E3EB68F94E2B

\title{
Studies in Liocranidae (Araneae): a new afrotropical genus featuring a synapomorphy for the Cybaeodinae
}

\author{
Jan BOSSELAERS ${ }^{1} \&$ Rudy JOCQUÉ ${ }^{2}$ \\ ${ }^{1,2}$ Royal Museum for Central Africa, Tervuren, Belgium \\ ${ }^{2}$ Email: jocque@africamuseum.be (corresponding author) \\ ${ }^{1}$ urn:1sid:zoobank.org:author:D6AD7414-3540-4F06-8631-8873450AA90C \\ ${ }^{2}$ urn:Isid:zoobank.org:author:CF15016C-8CD1-4C9D-9021-44CA7DC7A5D5
}

\begin{abstract}
Cteniogaster, a new genus of small ground spiders is described from Kenya and Tanzania. It encompasses seven new species, three of which are known from both sexes: $C$. toxarchus sp. nov., the type species, C. conviva sp. nov. and C. hexomma sp. nov. Three species are known from females only: C. lampropus sp. nov., $C$. sangarawe sp. nov. and $C$. taxorchis sp. nov. and one only from males: $C$. nana sp. nov. The new genus can be recognised by the presence of a posterior ventral abdominal field of strong setae and anterior lateral spinnerets with enlarged piriform gland spigots in males. A cladistic analysis attributes the genus to Liocranidae, Cybaeodinae. The results of the analysis performed do not produce an unequivocal autapomorphy for Liocranidae, but provide a combination of non-homoplasious character changes that offers significant potential for recognising genera as Liocranidae. Moreover, robust apomorphies are determined within Liocranidae for the subfamilies Liocraninae and Cybaeodinae. Based on these findings Toxoniella Warui \& Jocqué, 2002 is transferred from Gallieniellidae to Liocranidae, Cybaeodinae. Jacaena Thorell, 1897, Plynnon Deeleman-Reinhold, 2001 and Teutamus Thorell, 1890 are transferred to Corinnidae, Phrurolithinae and Montebello Hogg, 1914 to Gnaphosidae. Itatsina Kishida, 1930 is synonymised with Prochora Simon, 1886.
\end{abstract}

Keywords. phylogeny, enlarged piriform gland spigots, ventral abdominal setae, eye reduction, Eastern Arc Mountains.

Bosselaers J. \& Jocqué R. 2013. Studies in Liocranidae (Araneae): a new afrotropical genus featuring a synapomorphy for the Cybaeodinae. European Journal of Taxonomy 40: 1-49. http://dx.doi.org/10.5852/ejt.2013.40

\section{Introduction}

Liocranidae Simon, 1897 feature among the poorly defined spider families (Jocqué \& DippenaarSchoeman 2006) since not a single synapomorphy has been listed so far. Making abstraction of the Paratinae (Marusik et al., 2008) which are only tentatively placed in Liocranidae, both subfamilies that compose the family, Cybaeodinae Simon, 1893 and Liocraninae Simon, 1897, are also poorly delimited. The present paper describes a new genus in which the males are characterized by a conspicuous group of rigid setae on the venter of the adomen. More important is that they have enlarged piriform gland spigots on the male anterior lateral spinnerets, a character first described by Platnick (1990) for some Clubionidae Wagner, 1887, and later also discovered in Toxoniella Warui \& Jocqué, 2002, a genus that was attributed 
to Gallieniellidae Millot, 1947. This peculiar spinneret character appears to be present in most genera (Agroeca Westring, 1861, Agraecina Simon, 1932, Cteniogaster gen. nov., Cybaeodes Simon, 1878, Liocranoeca Wunderlich, 1999, Neoanagraphis Gertsch \& Mulaik, 1936) that have been attributed to the Cybaeodinae, but is lost in Apostenus Westring, 1851 and Scotina Menge, 1873. Nevertheless, a cladistic analysis shows that its presence can be considered a synapomorphy for the subfamily. Apart from the description of the new genus, the relationships between the genera in Liocranidae and the distribution of the spinneret character on the cladogram are studied in the paper.

\section{Material and Methods}

Specimens were observed and drawn using Euromex MIC465 and Olympus SZX9 binocular microscopes. Photographs of the habitus were taken with a Leica MZ16 binoclar microscope using the LAS automontage software. Female epigynes were detached from the abdomen, temporarily mounted in a clearing mixture of methyl salicylate and cedukol (Merck, Darmstadt) and observed with a Leitz Dialux 22 microscope and subject to automontage with the Syncroscopy software. For SEM photos, specimens or parts were dried in HMDS, gold coated and examined and photographed with a JEOL $6480 \mathrm{LV}$ scanning electron microscope. Tarsal claws and details of the distal end of the male bulbus were observed and drawn using a Wild M12 compound microscope, except for the tarsal clams of Apostenus spinimanus (Koch \& Berendt, 1854), for which a fossil in Baltic amber (CJB) was observed with an Olympus SZX9 binocular microscope. In order to precisely position bulbi for observation under the compound microscope (Fig. 11A, B), the male palps were immobilized in Schwarzkopf "Freezing Gel Extreme 5", a transparent vinylpyrrolidone-vinylacetate copolymer (CAS 25086-89-9) / carbomer gel (Schröder et al. 2000; Shulze zur Wiesche 2006). Leg spination is illustrated in a schematic representation (Appendix 1) where pl, do, $\mathrm{rl}$ and ve sides of leg articles are flattened as a folding net (Dürer 1525). The format for leg spination in the genus description follows Platnick \& Shadab (1975), amended for ventral spine pairs according to Bosselaers \& Jocqué (2000). All measurements are in mm.

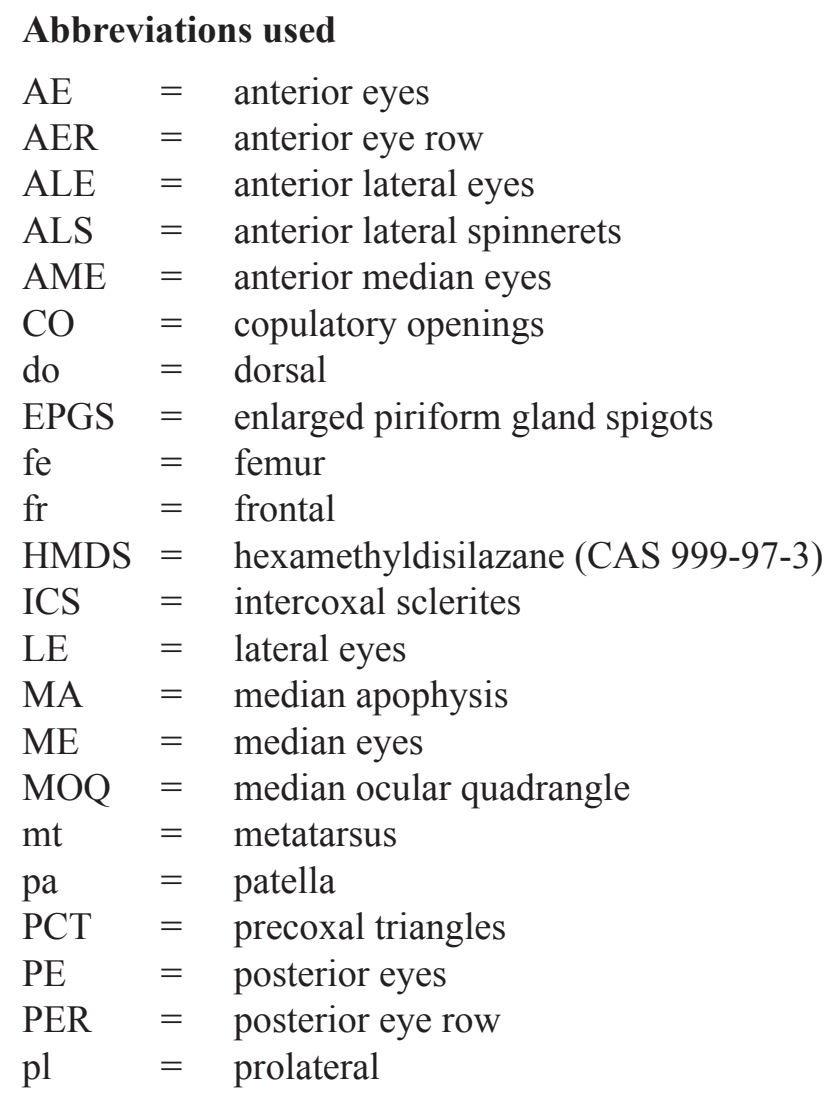


BOSSELAERS J. \& JOCQUÉ R., A new afrotropical genus of Cybaeodinae (Araneae, Liocranidae)

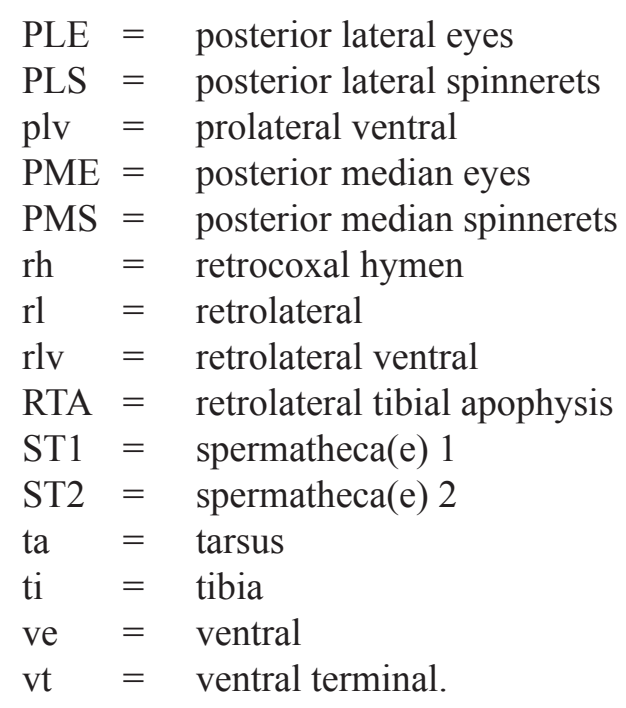

Abbreviations of personal and institutional collections (curators in parentheses)

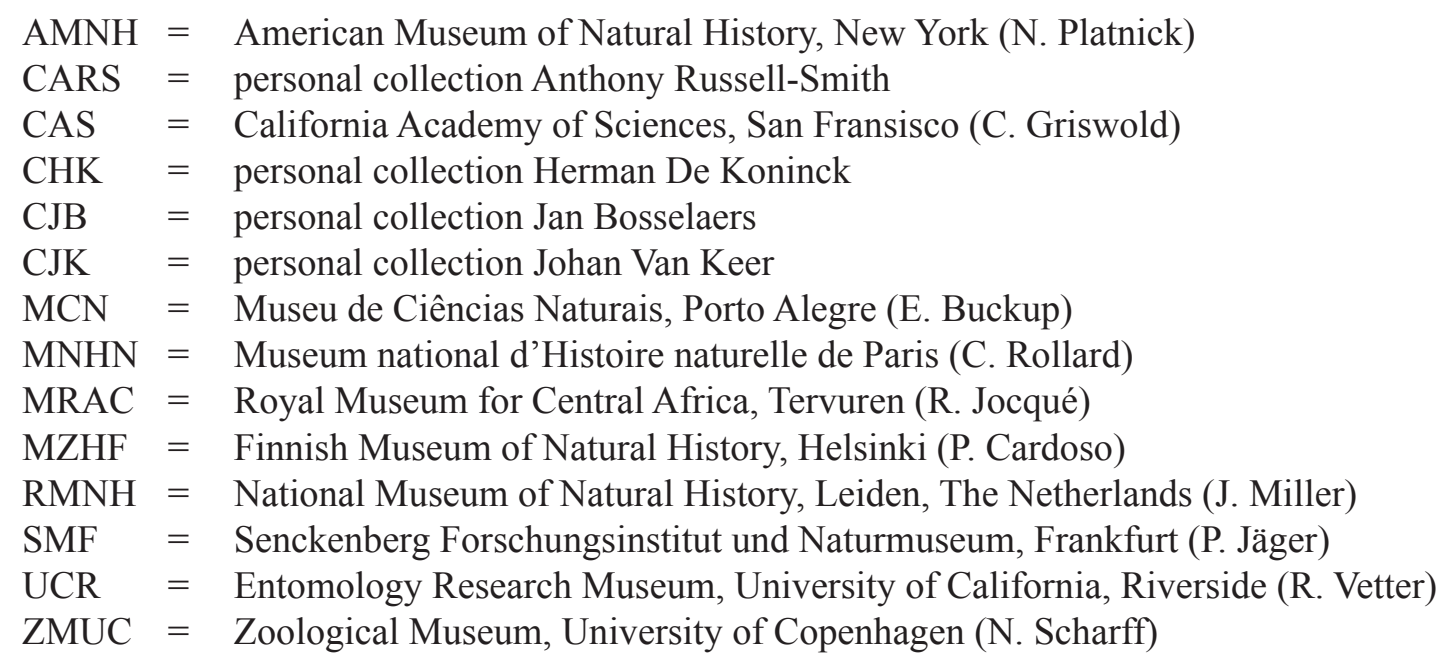

Phylogenetic analyses were performed using the computer programmes PAUP* 4.0 beta 10 (Swofford 2002), Winclada 1.00.08 (Nixon 2002), and TNT 1.1 (Goloboff et al. 2003, 2008a). Optimisation of character states and printing of the preferred tree was performed using Winclada. All three programmes were run on a dual-core Intel iMac under a Windows XP virtual machine (VMware Fusion 2, VMware, Inc., 3401 Hillview Avenue, Palo Alto, CA 94304, USA; Bugnion et al. 2000).

\section{Taxon choice}

Based on somatic and genitalic characters, Cteniogaster gen. nov. was tentatively attributed to Liocranidae. In order to investigate its relationships in a more objective way, a phylogenetic analysis was performed. The analysis included the three species of Cteniogaster gen. nov. of which males and females are available, 23 species of Liocranidae belonging to 11 different genera, and the two known species of Toxoniella Warui \& Jocqué, 2002, a genus similar to Cteniogaster gen. nov., whose current place in Gallieniellidae is perceived as uncertain. Where possible, more than one species was included for each genus, in order to increase the robustness of the analysis. Two corinnid species, Corinna nitens (Keyserling, 1891) and Creugas bajulus (Gertsch, 1942), were used as outgroup (Watrous \& Wheeler 1981; Maddison et al. 1984).

A list of material examined for assessment of morphological characters is provided in Appendix 2. 


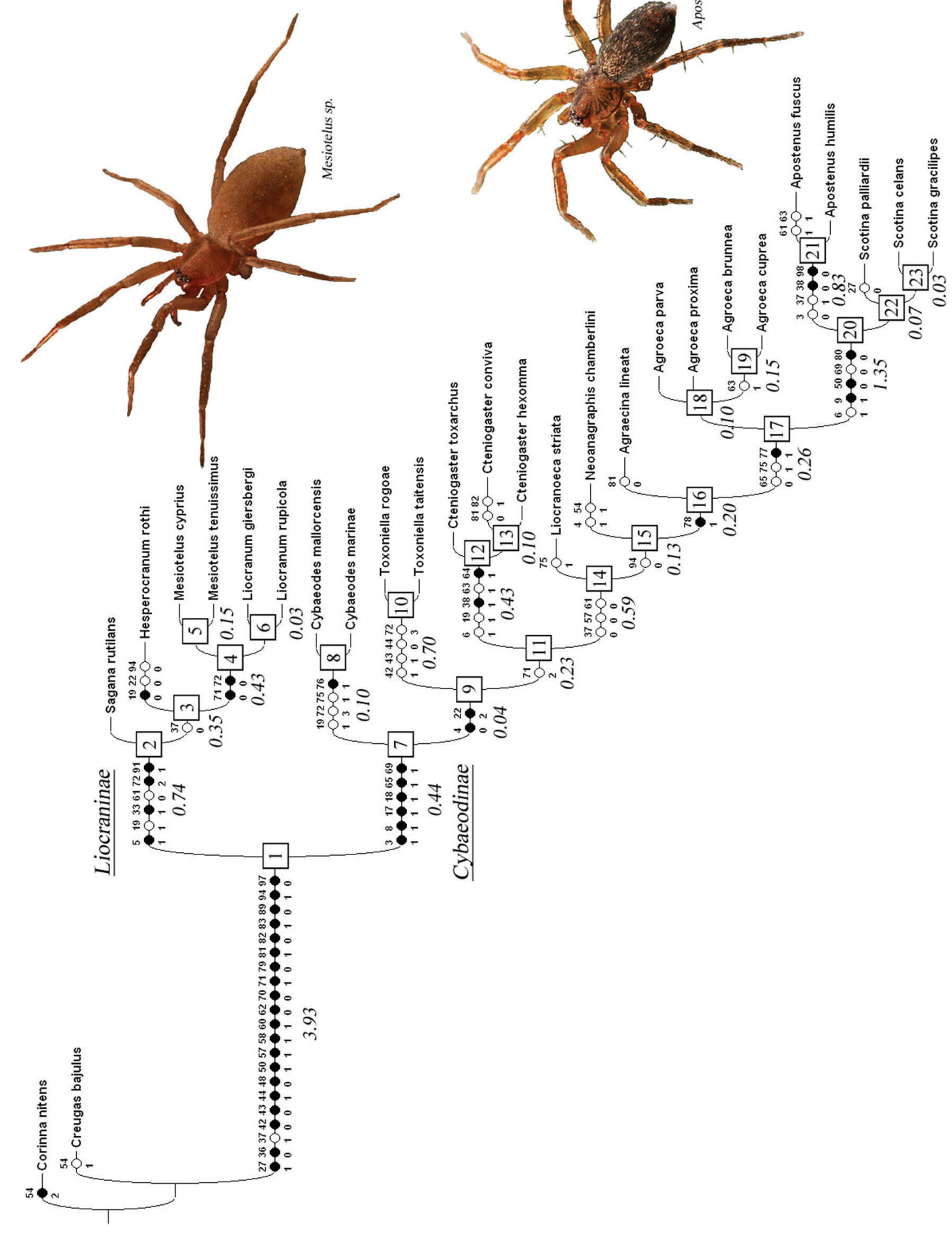

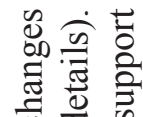

劳

क

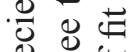

क⿺ ⿻肀口

응 글응

흘

可

:

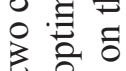

苛

का है

品

की 卷

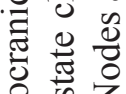

呈 क

弪 $\frac{0}{0}$

क⿺ 万ٓ

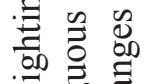

3.50

उ छे

志式 跑

可 $\frac{0}{3}$

离竞

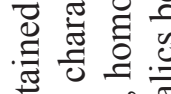

。ัँ

记

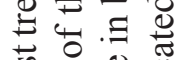

总言离:

守密

三 흥

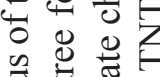

号芯寻

过 气 :

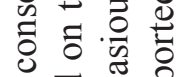

멍 융

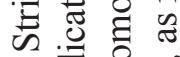

$\rightarrow$ 들

离范艺苋 


\section{Characters}

A series of 99 characters ( 77 binary and 22 multistate) was coded for the 27 taxa chosen. All characters are phylogenetically informative. Where possible, characters were scored with character states hierarchically related, as advocated by Hawkins et al. (1997), even though this necessitated coding missing entries due to character inapplicability in some instances (Maddison 1993). Genitalic traits are dealt with in 26 of the characters, the other 73 characters are somatic, 21 of them referring to leg or palp spination. Possible problems of spine homology were addressed as in Bosselaers (2002: 143) and Bosselaers \& Jocqué (2002: 244). All 99 characters used in the analysis are explained below. On the preferred tree (Fig. 1), 29 characters can be optimised ambiguously (indicated by ${ }^{\mathrm{A}}$ in the list below), and 26 characters are fully non-homoplastic (indicated by an asterisk). Out of the 99 characters scored, 47 are shown in Fig. 1 (indicated by ${ }^{\mathrm{T}}$ in the list below).

1. AMale rh: (0) absent, (1) present. The rh was first described by Raven (1998) as the "retrocoxal window". It is a weak spot, in most cases hyaline and lens-shaped, on the rl face of coxa I (Bosselaers \& Jocqué 2002: fig. 1A).

2. ${ }^{\mathrm{A}}$ Female retrocoxal hymen: (0) absent, (1) present.

3. ${ }^{\mathrm{T}}$ Trochanter notch: (0) absent, (1) present.

4. ${ }^{\mathrm{T}}$ Rows of bristles in ve scopulae of legs I and II: (0) absent, (1) present.

5. ${ }^{* \mathrm{~T}}$ Rows of bristles in ve scopulae of legs I and II: (0) small, (1) large, erectile. The bristles mentioned under character 4 can be small, i.e. hardly larger than normal scopulae, or large and erectile, and with a peculiar basal socket, as described by Ubick \& Platnick (1991: 2).

6. ${ }^{\mathrm{T}}$ Patellar indentation of legs I and II: (0) narrow, (1) wide. The patellar indentation is a slit-like membranous groove on the rl side of the pa (Simon 1892: 22; Ledoux \& Canard 1991: 9, fig. 15A, B)

7. Patellar indentation of leg IV: (0) narrow, (1) wide.

8. ${ }^{* \mathrm{~T}}$ Bent male tarsi IV: (0) absent, (1) present (Fig. 11D). The tarsi IV of males are bent and subsegmented in a characteristic way in a number of liocranid genera (Ubick \& Vetter 2005: 71; Wunderlich 1999: 68).

9. * ${ }^{\mathrm{T}}$ Femur I plv spines: (0) absent, (1) present.

10. Femur I apical do spine: (0) absent, (1) present.

11. Femur III and IV apical do spine: (0) absent, (1) present.

12. Patella III and/or IV rl spine: (0) absent, (1) present.

13. ${ }^{A}$ Male ti I plv spines: (0) absent, (1) one to three, (2) four to six, (3) more than six.

14. AFemale ti I plv and rlv spines: (0) absent, (1) one to three, (2) four to six, (3) more than six.

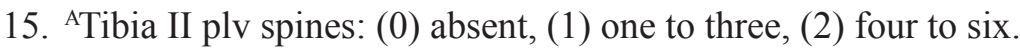

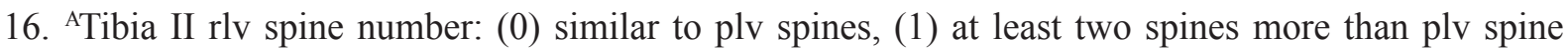
number.

17. ${ }^{* T}$ Female ti III do spines: (0) absent, (1) present.

18. ${ }^{* \mathrm{~T} T}$ Tibia IV do spines: (0) absent, (1) present.

19. ${ }^{\text {TA }}$ Metatarsus I and II ve spine pairs: (0) absent, (1) one, (2) more than one.

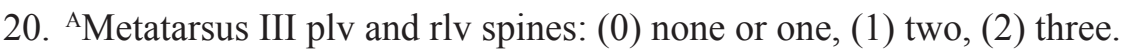

21. ${ }^{\mathrm{A}}$ Metatarsus IV plv and rlv spines: (0) absent, (1) one, (2) two, (3) three.

22. ${ }^{\text {TA Male }} \mathrm{mt}$ IV rl spines: (0) one or two, (1) three to four, (2) five or more.

23. Metatarsus III and IV vt spines: (0) absent, (1) present on both.

24. Male palpal pa pl spines: (0) absent, (1) present. 
25. AMale palpal ti pl spines: (0) none or one, (1) two, (2) three, (3) four.

26. Male palpal ti do spines: (0) absent, (1) one, (2) two.

27. ${ }^{\mathrm{T}}$ Male palpal ta distal pl edge spines: (0) absent, (1) present.

28. Female palpal fe pl spines: (0) absent, (1) present.

29. Female palpal ti pl spines: (0) two, (1) three, (2) four.

30. ${ }^{A}$ Feathery hairs (Griswold 1993: fig. 61; Townsend \& Felgenhauer 1998: fig. 8): (0) absent, (1) present (Fig. 6B).

31. Metatarsal vt preening brush on tarsi III and IV: (0) absent, (1) present.

32. ${ }^{\mathrm{A}}$ Metatarsal vt preening brush on tarsi III and IV: (0) sparse, (1) dense.

33. ${ }^{* T}$ Tibia I and II ve scopulae: (0) absent, (1) present.

34. Metatarsal ve scopulae: (0) absent, (1) present.

35. Tarsal ve scopulae: (0) absent, (1) present.

36. ${ }^{* \mathrm{~T}}$ Claw tufts: $(0)$ absent, (1) present.

37. ${ }^{\mathrm{T}}$ Tenent hairs (Forster 1970: 18; Ubick \& Vetter 2005: 69, 71): (0) absent, (1) present (Figs 6H, 11C-E).

38. *TATenent hair pairs: (0) one, (1) two, (2) four or more. The number of tenent hair pairs present varies considerably between liocranid genera (Bosselaers 2009: 39, 49, 51), as will be discussed below.

39. PCT: (0) absent, (1) present. PCT are small triangular sclerites surrounding the sternum, their tips facing the bases of the coxae (Penniman 1985: 16).

40. ICS in male: (0) absent, (1) two pairs, between coxae I and II and between coxae II and III, (2) three pairs. ICS are small triangular or elongated sclerites surrounding the sternum, their tips penetrating between the coxae of the legs.

41. Antercoxal sclerites in female: (0) absent, (1) two pairs, between coxae I and II and between coxae II and III, (2) three pairs.

42. 'Sternal border: (1) simple or with indistinct, thin border, (2) strongly rebordered.

43. TRetromarginal cheliceral teeth number: (0) two, (1) more than two.

44. Thong shaggy hair in front of fang base: (0) absent, (1) present. A conspicuous hair at the cheliceral promargin, as long as the fang and bent at a right angle just beyond its base (Bosselaers \& Jocqué 2002: fig. 1M,Q; Platnick 2000: 10).

45. ${ }^{A}$ Chilum: (0) absent, (1) present. The chilum is a small subtriangular sclerite at the base of the chelicerae, below the clypeus (Jocqué 1991: 11).

46. Chilum: (0) bilateral and bipartite, (1) median and entire.

47. ${ }^{\mathrm{A}}$ General shape of endites: (0) with external lateral notch, (1) parallel-sided.

48. ${ }^{* \mathrm{~T}}$ Serrula of endites: $(0)$ conspicuous, (1) reduced.

49. AApical maxillar hair tuft of endites: (0) absent, (1) present.

50. ${ }^{* T}$ Carapace shape in lateral view: (0) carapace slanting, highest at fovea, (1) carapace flat, (2) carapace bulging, highest in cephalic region.

51. Clypeus height: (0) smaller than diameter of AME, (1) equal to diameter of AME, (2) larger than diameter of AME.

52. Male AER curvature from front: (0) procurved, (1) straight.

53. ${ }^{\mathrm{A}}$ Male PER curvature, do view: (0) procurved, (1) straight, (2) recurved.

54. ${ }^{\mathrm{T}} \mathrm{AME}$ size: (0) smaller than ALE, (1) equal to ALE, (2) larger than ALE.

55. PME size: (0) smaller than PLE, (1) equal to PLE. 
BOSSELAERS J. \& JOCQUÉ R., A new afrotropical genus of Cybaeodinae (Araneae, Liocranidae)

56. AAME size: (0) smaller than PME, (1) equal to PME, (2) larger than PME.

57. ${ }^{\mathrm{T}} \mathrm{PME}$ shape: (0) circular, (1) modified.

58. ${ }^{* \mathrm{~T} M O Q:(0) ~ w i d e r ~ a n t e r i o r l y, ~(1) ~ w i d e r ~ p o s t e r i o r l y . ~}$

59. *APE interdistance: (0) equidistant, interdistance larger than PE diameter, (1) equidistant, interdistance smaller than PE diameter, (2) PME farther from each other than from PLE, interdistance PME-PLE smaller than PE diameter.

60. ${ }^{* \mathrm{~T}}$ Curved strong hairs frontally on abdomen: (0) absent, (1) present. Many ground spider genera have rows of strong curved hairs frontally on the abdomen (Bosselaers \& Jocqué 2002: fig. 2D).

61. TPartial male do abdominal scutum: (0) absent, (1) present.

62. *TMale epigastric sclerite: (0) absent, (1) present.

63. ${ }^{\mathrm{T}}$ Male modified ve abdominal setae: (0) absent, (1) present. Several liocranid genera have males with a patch of stout, modified ve abdominal setae (Ubick \& Vetter 2005: 71; Wunderlich 1999: 68, 2004: 1624, 2008: 489, 2011: 120, fig. 17a, 2012: 128).

64. ${ }^{* \mathrm{~T}}$ Male modified ve abdominal setae field: (0) large, (1) small (Figs 2P, 3E, 6D, 9B, 10B).

65. ${ }^{\mathrm{T}}$ Male ALS shape: (0) conical, (1) cylindrical (Figs 7E, 9B, 10B).

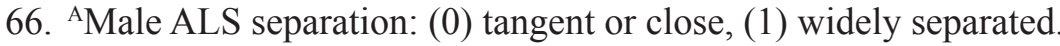

67. Male PMS shape: (0) slender, (1) stout, subtriangular (Fig. 7F).

68. Male PLS separation: (0) about half of PLS length, (1) at least equal to PLS length.

69. ${ }^{\mathrm{T}}$ Enlarged piriform spigots on male ALS: (0) absent, (1) present (Fig. 7E). Enlarged piriform gland spigots on ALS of males only were first described by Platnick for Clubiona Latreille, 1804 and Elaver O. P.-Cambridge, 1898 (Platnick 1990: 35), and later by Platnick \& Di Franco (1992: 4-6) also for Cybaeodes, Agroeca, Neoanagraphis, and Rhaeboctesis Simon, 1897. Bosselaers \& Jocqué (2002: char. 112, fig. 2KL) also mention them for Agraecina and Liocranoeca. Warui \& Jocqué (2002: 307) mention the character for Toxoniella. Wunderlich attributes the character to Cybaeodes and Clubioninae Simon, 1897 (2004: 1625, 2011: 27), and wrongly mentions it for Apostenini Wunderlich, 2008 (2008: 488, but compare Ubick \& Vetter 2005: 69, fig. 15).

70. ${ }^{* T}$ Female epigastric sclerite: (0) absent, (1) present.

71. TFemale PMS shape: (0) slender, (1) laterally compressed, (2) stout, subtriangular (Fig. 7D).

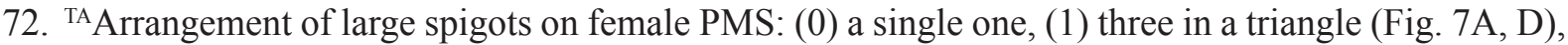
(2) five in two rows, (3) more than five in two rows.

73. Female PLS separation: (0) about half of PLS length, (1) at least equal to PLS length.

74. Male cymbial tip: (0) wide and short, (1) narrowed and long.

75. ${ }^{\mathrm{T}}$ Dorsal bristle mat on male palpal cymbium: (0) absent, (1) present. A do palpal bristle mat was mentioned for Zoropsidae Bertkau, 1882 by Levy (1990: fig. 7) and Bosselaers (2002: 145) It occurs in many families, for example in several corinnid genera.

76. ${ }^{* \mathrm{~T}}$ Dorsal palpal bristle mat size: (0) large, (1) small.

77. ${ }^{* \mathrm{~T}}$ Tegular locking lobe: (0) absent, (1) present. Described by Griswold (1993: 1, fig. 19) for Lycosoidea Lehtinen, 1967 as a tegular lobe interlocking with a corresponding lobe on the subtegulum. In Liocranidae, Agraecina lacks the tegular lobe but has the subtegular one.

78. *TSubtegular locking lobe: (0) absent, (1) present.

79. ${ }^{* \mathrm{~T} C o i l e d ~ s p e r m ~ d u c t: ~(0) ~ a b s e n t, ~(1) ~ p r e s e n t . ~ A ~ c o i l e d ~ s p e r m ~ d u c t ~ i n ~ t h e ~ b u l b u s ~ i s ~ a t t r i b u t e d ~ t o ~}$ Corinninae Karsch, 1897 by Platnick \& Baptista (1995: 5).

80. ${ }^{* \mathrm{~T}}$ Conductor: (0) absent, (1) present.

81. ${ }^{\mathrm{T}}$ Conductor: (0) sclerotised, (1) membranous. 

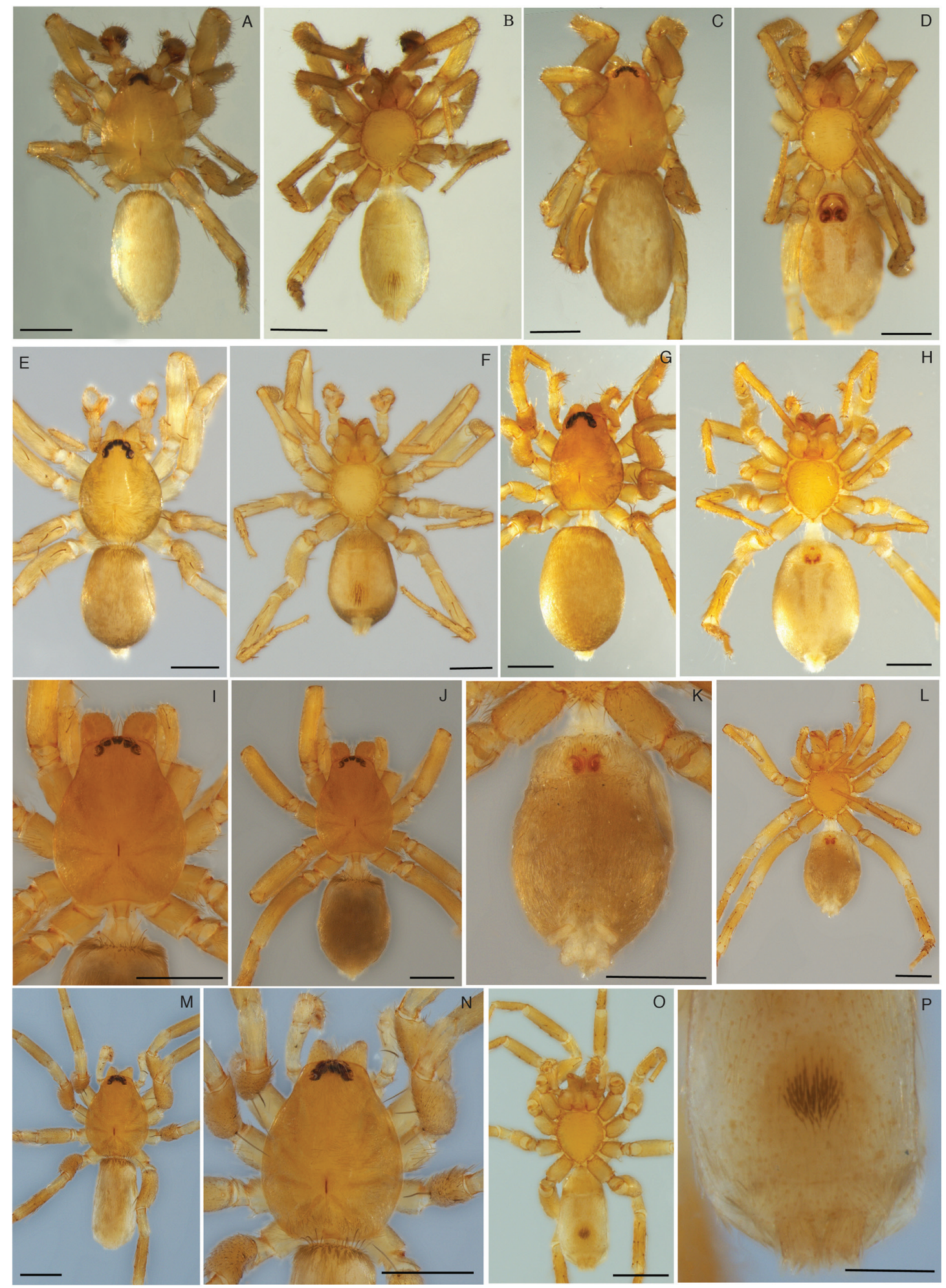

Fig. 2. A-D. Cteniogaster conviva sp. nov. A. đ, habitus dorsal view. B. Idem, ventral view. C. + , habitus, dorsal view. D. Idem, ventral view. E-H. Cteniogaster toxarchus gen. et sp. nov. E. $\widehat{\jmath}$, habitus dorsal view. F. Idem, ventral view. G. $q$, habitus, dorsal view. H. Idem, ventral view. I-P. Cteniogaster hexomma sp. nov. I. , , prosoma, dorsal view. J. Idem, habitus, dorsal view. K. Idem, abdomen, ventral

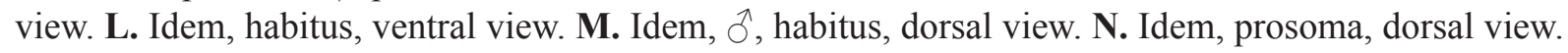
O. Idem, habitus, ventral view. P. Idem, abdomen, ventral view. Scale bars: $\mathrm{A}-\mathrm{O}=0.5 \mathrm{~mm} ; \mathrm{P}=0.2 \mathrm{~mm}$. 
82. ${ }^{\mathrm{T}}$ Conductor shape: (0) simple, (1) complex.

83. ${ }^{* \mathrm{~T}} \mathrm{MA}$ : (0) absent, (1) present. The MA is a sclerotised, articulated tegular process arising from a membranous area (Bonaldo 1997: 166; Griswold 1993: 10, char. 12; Sierwald 1990: 21).

84. MA shape: (0) simple hook, (1) curled and bifid.

85. Embolus insertion: (0) apical, (1) prolateral.

86. ${ }^{\mathrm{A}}$ Embolus transsection: (0) solid, (1) flattened.

87. AEmbolus shape: (0) a short point, (1) sickle-shaped, (2) platelike, (3) hook-shaped, (4) a flat ribbon, (5) whip-shaped.

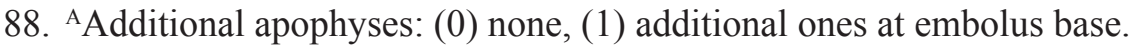

89. *TSubtegulum: (0) pl, (1) pl and rl.

90. ${ }^{\text {AB }}$ asal rl bulge with thickened rim on cymbium: (0) absent, (1) present.

91. *TProlateral terminal lobe on male palpal ti: (0) absent, (1) present. Such a lobe (Bosselaers \& Jocqué 2002: fig. 4H) was mentioned by Ubick \& Platnick (1991: 11) for Liocranum L. Koch, 1866, Mesiotelus Simon, 1897, and Hesperocranum Ubick \& Platnick, 1991.

92. Epigynal sclerotisation: (0) weak, (1) substantial.

93. Epigynal scape: (0) absent, (1) present.

94. ' Epigynal anterior hood (Bosselaers \& Jocqué 2002: fig. 4A, C, F): (0) absent, (1) present.

95. AEpigynal septum: (0) absent, (1) present.

96. Lateral epigynal hooks: (0) absent, (1) present.

97. *TEpigyne CO position: (0) anterior, (1) posterior.

98. ${ }^{* \mathrm{~T} S T 2:}$ (0) absent, (1) present. For a discussion of ST2, see Bosselaers \& Jocqué (2000: 15, char. 84).

99. ST2 size: (0) smaller than ST1, (1) larger than ST1.

\section{Results}

The matrix of character states, as well as the character parameters on the preferred tree, can be found in Appendix 3. All characters were run unordered in the analyses performed.

In order to avoid ambiguous results introduced by species with many missing entries, the data matrix includes only species of which males and females were available. An equally weighted analysis of the data matrix was performed in PAUP with hsearch addseq=random nreps $=\mathbf{1 0 0 0 0}$ (heuristic search with tree bisection and reconnection swapping and 10000 random addition sequences). In order to avoid spurious resolution due to unsupported (Coddington \& Scharff 1994; Wilkinson 1995) or ambiguously supported (Nixon \& Carpenter 1996) branches, those with a minimum length of zero were collapsed with condense collapse $=$ minbrlen. Thirty-one shortest trees of length 312 were found, belonging to two tree islands of 19 and 12 trees, respectively. The strict consensus of these 31 trees is fully compatible with but less resolved than the preferred tree in Fig. 1.

Because cladograms obtained by attributing a posteriori weights to characters based on their relative degrees of homoplasy on a set of heuristic trees explain the data better (Bosselaers \& Jocqué 2002; Goloboff et al. 2008b), a weighted analysis was also performed. Implied weighting (Goloboff 1993) was preferred for this purpose. When applying implied weighting in PAUP with pset goloboff $=\mathbf{y e s}$, hsearch addseq $=$ random nreps $=\mathbf{5 0 0 0}$, collapsing branches with a minimum length of zero with condense collapse $=$ minbrlen, three fittest trees with fit $=-68.50714$ (PAUP attributes a negative sign to fit values) were found. The default value for the concavity constant was used, which equals 2 in PAUP, since that programme uses a concavity constant $k$ as defined in the fit formula $f_{i}=(k+1) /\left(s_{i}+k\right.$ 
$+1-\mathrm{mi})=(\mathrm{k}+1) /(\mathrm{es}+\mathrm{k}+1)$ in Goloboff $(1993)$. An additional implied weighting search run in TNT using Settings / implied weighting and Analyze / Traditional search as well as New Technology search (concavity constant $=3$ ) produced another fit value (30.49286) for the same trees, because TNT calculates weighted homoplasy (a complement of fit) for implied weighting trees. The strict consensus of the three fittest trees also has fit $=-68.50714$, length $=313, \mathrm{ci}=0.412, \mathrm{ri}=0.725, \mathrm{hi}=0.588$, and $\mathrm{rc}$ $=0.299$. This tree is our preferred solution (Fig. 1).

Bremer support values (Bremer 1988, 1994) for the preferred tree, expressed as fit values, were calculated in TNT using Analyze / suboptimal, followed by Analyze / Traditional search / tree bisection reconnection (TBR) and Trees / Bremer Supports, retaining trees suboptimal up to 10 units of fit and combining various numbers of replications (between 1 and 10) with various numbers of trees saved per replication (between 3000 and 30000, inversely related to the numbers of replications) until the solution stabilised.

Taxonomy

\author{
Class Arachnida Cuvier, 1812 \\ Order Araneae Clerck, 1757 \\ Family Liocranidae Simon, 1897 \\ Subfamily Cybaeodinae Simon, 1893 \\ Cteniogaster gen. nov. \\ urn:1sid:zoobank.org:act:4D38E26E-FE0F-4FD9-ADB2-9B4728BE56AD
}

\title{
Type species
}

Cteniogaster toxarchus sp. nov.

\section{Diagnosis}

Cteniogaster gen. nov. differs from all other genera of Liocranidae by the presence of a small posterior ve abdominal field of strong setae in males, the presence of two pairs of tenent hairs on the tarsal tips and the consistent presence of one plv and one rlv spine on $\mathrm{mt} \mathrm{IV}$. The genus differs from all other liocranid genera except Apostenus by the wide patellar indentations.

\section{Etymology}

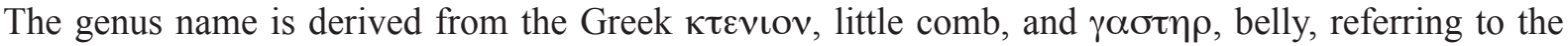
small array of strong, modified ventral setae on the abdomen of the males of the new genus. The gender is feminine.

\section{Description}

Small (males 1.7-3.0, females 2.0-3.0) spiders. Carapace longer than wide, almost flat but slightly higher at fovea (Fig. 9F, G), smooth (Fig. 6A), yellow or brown, covered with feathery hairs (Fig. 6B), unicoloured or with faint pattern, iridescent in some species. Distinct fovea in posterior half. MOQ widest posteriorly (Fig. 9H). Eight, six or four eyes, ringed with black. AME dark, LE pearl, PME very pale and in most species reduced, or absent. AME smaller than LE. Both eye rows straight in frontal view (Fig. 12E). Chilum single, small and subtriangular, indistinct or absent in the smaller species. Chelicerae small, yellow or brown, with a knee-shaped shaggy hair in front of fang base. Promarginal cheliceral rim with three teeth at a small distance from fang base, largest one in the middle. Retromarginal cheliceral rim with two small teeth close to fang base. Labium subtriangular, about as wide as long, with a thickened anterior rim. Endites with a lateral notch and an apical serrula, no apical hair tuft. Sternum 
BOSSELAERS J. \& JOCQUÉ R., A new afrotropical genus of Cybaeodinae (Araneae, Liocranidae)
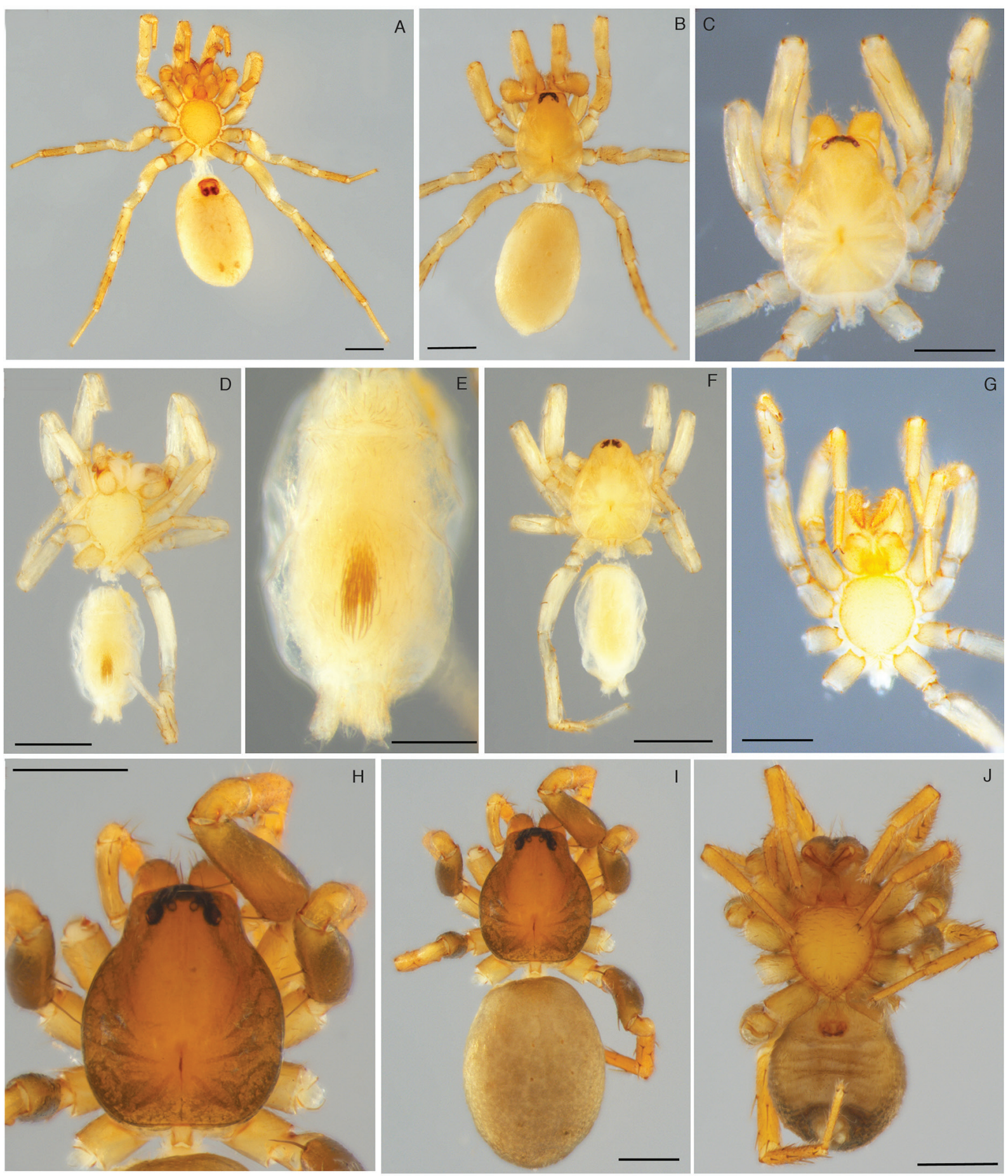

Fig. 3. A-B. Cteniogaster sangarawe sp. nov. A. + , habitus, ventral view. B. Idem, dorsal view. C-D. Cteniogaster taxorchis sp. nov. C. , , prosoma, dorsal view. D. Idem, ventral view. E-G. Cteniogaster nana sp. nov. E. O, habitus, ventral view. F. Idem, abdomen, ventral view. G. Idem, habitus, dorsal view. H-J. Cteniogaster lampropus sp. nov. H. , prosoma, dorsal view. I. Idem, habitus, dorsal view. J. Idem, ventral view. Scale bars: A-D, F-J $=0.5 \mathrm{~mm} ; \mathrm{E}=0.2 \mathrm{~mm}$. 
shield-shaped, smooth, unicoloured yellow or brown, in some species with a thin border. Three pairs of weak PCT present, ICS absent.

Abdomen in different shades of grey, with an anterior row of curved strong hairs in both sexes (Figs $2 \mathrm{~N}$, 9A), and with broad, flattened feathery hairs on ve surface (Fig. 6E, F). The male abdomen has a small and weak anterior do scutum and a small ve posterior patch of strong setae (Figs 2B, F, P, 3E, 6D, E, 9B, 10B). Males with ALS cylindrical and separated from each other, bearing apical EPGS (Fig. 7E), PMS and PLS thin and slender, PLS separated by more than their length and having a dome-shaped apical segment (Fig. 7E, F). Females with non-contiguous cylindrical ALS with one major ampullate and a small number of unmodified piriform gland spigots, subtriangular PMS with three cylindrical and one minor ampullate gland spigot and non-contiguous subcylindrical PLS about as large as ALS bearing two cylindrical gland spigots and about a dozen aciniform gland spigots (Fig. 7A-D). Legs pale yellow to brown, unicoloured, iridescent in some species, with trichobothria with basal socket (Fig. 6G) and oval tarsal organ (Fig. 6I). Patellar indentation wide, length $2 / 3$ of pa length in legs I to III, $1 / 2$ of patella length in leg IV. Retrocoxal hymen present, but often pale and weak. Trochanter notch present. Tarsus IV bent with pseudoarticulations in males (Fig. 11D), and slightly bent in females. Leg formula 4123. Tarsal tips without true claw tufts but with two pairs of tenent hairs (Figs 6H, 11C, E). Metatarsus IV with one plv and one rlv spine, both in proximal half of article (Appendix 1). Minimal leg spination for the genus: males fe: palp do 0-1-1; I pl 0-0-1, do 1-1-0; II do 1-1-0; III do 1-1-2; IV do 1-1-0; pa: palp do 0-1; ti: palp pl 2-0-1; I plv 1-0-1, rlv 1-0-1; II rlv 1-0-1; III pl 1-0-1, do 1-0-0, rl 0-0-1, plv 1-1-1, rlv $0-0-1$; IV pl 1-0-1, do 1-0-0, rl 1-0-1, plv 1-1-1, rlv 0-1-1; mt: I plv 1-0-0, rlv 1-0-0; II plv 1-0-0, rlv 1-00 ; III pl 0-0-1, do 0-2-2, rl 1-0-1, ve 2-0-1; IV pl 0-0-1, do 2-2-2, rl 1-0-1, ve 2-0-1; ta: palp pl 1-0-1, do 1-0-0; females fe: palp do 0-1-1; I pl 0-0-1, do 1-1-0; II do 1-1-0; III do 1-1-2; IV do 1-1-0; pa: palp pl 1-0, do 0-1; ti: palp pl 2-0-1, do 1-0-1; I plv 1-0-1, rlv 1-0-1; II rlv 1-0-1; III pl 1-0-1, do 1-0-0, rl 0-0-1, plv 1-1-1, rlv 0-1-1; IV pl 1-0-1, do 1-0-0, rl 1-0-1, plv 1-1-1, rlv 0-1-1; mt: I plv 1-0-0, rlv 1-0-0; II plv 1-0-0, rlv 1-0-0; III pl 0-0-1, do 0-2-2, rl 1-0-1, ve 2-0-1; IV pl 0-0-1, do 2-2-2, rl 1-0-1, ve 2-0-1; ta: palp pl 2-0-1, do 1-0-0, rl 0-1-0, ve 0-0-2.

Male palp with a simple, blunt or pointed RTA, a cymbium with a spine on the distal pl edge (Figs 9C, 10C), a pl subtegulum, a short apical embolus, a membranous or sclerotised conductor and a simple, subtriangular or subrectangular MA (Fig. 11A, B). Epigyne with an anterior hood and a central depression (Figs 9E, 10E, 12C-G, K-L). Vulva with isodiametric, oval or kidney-shaped ST1 with internal spikes and ST2 with a porous glandular structure (Figs 4, 5, 8).

\section{Distribution}

The genus is known from the Taita hills in Kenya and from the East and West Usambara mountains in Tanzania. So far no specimens of Cteniogaster gen. nov. have been found outside the Eastern Arc (Fig. 13), which is a fair indication that the genus is endemic to that mountain range.

\section{Identification Key}

Females

(not known for C. nana sp. nov.)

1. Frontal hood on epigyne large, wider than half epigyne width (Fig. 5) ....................................... 2

- Frontal hood on epigyne narrow, less than one fourth epigyne width (Fig. 4) ................................ 4

2. Frontal hood as large as half epigyne width; spermathecae 1 pear shaped, oriented diagonally, spermathecae 2 oriented transversally, touching in the middle (Fig. 5E-F) .... C. taxorchis sp. nov.

- Frontal hood almost as wide as epigyne; spermathecae 1 rounded, spermathecae 2 oriented along longitudinal axis (Fig. 5A-D) 
BOSSELAERS J. \& JOCQUÉ R., A new afrotropical genus of Cybaeodinae (Araneae, Liocranidae)

3. Frontal hood very wide and sclerotized; spermathecae 2 curved, slightly separated, not touching (Fig. 5C-D)

C. sangarawe sp. nov.

- Frontal hood less wide and hardly sclerotized; spermathecae 2 sinuous, touching in the middle (Fig. 5A-B)

C. conviva sp. nov.

4. Anterior hood in larger oval depression; spermathecae 1 piriform, widely separated (Fig. 4E-F) ....

C. lampropus sp. nov.

- Anterior hood not surrounded by oval depression; spermathecae 1 not piriform 5

5. Spermathecae 1 very large, oval, touching in the middle (Fig. 4C-D)

C. hexomma sp. nov.

- Spermathecae 1 much smaller, not touching (Fig. 4A-B)

C. toxarchus sp. nov.

\section{Males}

(not known for C. lampropus sp. nov., C. sangarawe sp. nov. and C. taxorchis sp. nov.)

1. Cymbium with retrolateral bulge; sclerotized conductor large and coiled; RTA large, hookshaped, sharp (Fig. 12A, B)

C. conviva sp. nov.

- Without cymbial bulge; conductor small; RTA less large or straight or blunt (Figs 9C-D, 10C-D, 12A-B)

2. RTA very short, sharp and straight (Fig. 10C-D)

C. hexomma sp. nov.

- RTA longer and blunt or curved upwards (Figs 9C-D, 12H-I) 3

3. RTA straight, obliquely truncated; conductor membranous (Fig. 9C-D)

C. toxarchus sp. nov.

- RTA curved upwards, truncated tip; conductor sclerotized (Fig. 12H-I) C. nana sp. nov.

\section{Cteniogaster toxarchus sp. nov. urn:Isid:zoobank.org:act:CBA4D351-0360-4F20-A6ED-CF4CCB6E7BBB}

Figs 2E-H, 4A-B, 8D-F, 9, 11A, E, 14; Appendix 1C, F

\section{Diagnosis}

Cteniogaster toxarchus sp. nov. differs from all other Cteniogaster gen. nov. species by the male palp with a straight, blunt, subtrapezoidal RTA and a pointed subtriangular $\mathrm{rl}$ apical MA with a pl membranous border, and by the vulva with widely separated, kidney-shaped median ST1.

\section{Etymology}

The species name is a noun in apposition and is derived from the Greek $\tau 0 \xi \alpha \rho \chi 05$, commander of the archers, referring to the array of arrow-like setae on the posterior ve part of the male abdomen in Cteniogaster gen. nov., and to the present species' status as typus of the genus.

\section{Type material}

\section{Holotype}

O: TANZANIA, E. Usambara Mts., Kwamkoro Forest Reserve, 5¹0.9’S 38³5.8’E, 6 Nov. 1995, $950 \mathrm{~m}$ asl, Griswold C., Scharff N., Ubick D. (ZMUC).

\section{Paratypes}

4 우 3 j.: same data as holotype; 17 $\widehat{\jmath}, 25$ 우, 5 j.: TANZANIA, E. Usambara Mts., Amani, 5⒌7’S 38³8'E, 28 Oct.-9 Nov. 1995, $950 \mathrm{~m}$ asl, sifting litter, Griswold C., Scharff N., Ubick D. (ZMUC);

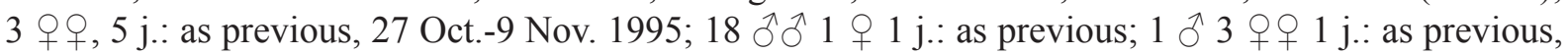

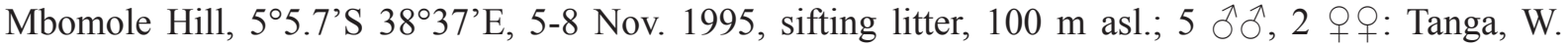
Usambara Mts., Mazumbai Forest, 449'S 38³0'E, 11-19 Nov. 1995, pitfall traps, 1400-1600 m asl, 


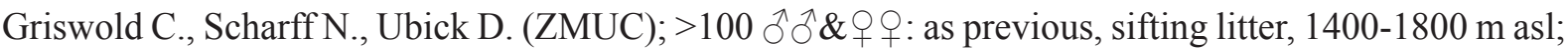

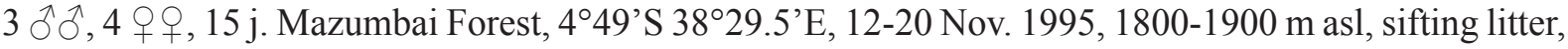

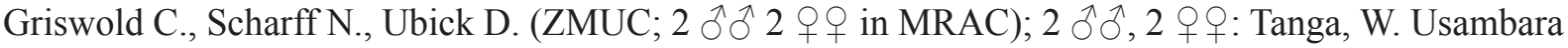
Mts., Mazumbai Forest, 449’S 38³0'E, 11-20 Nov. 1995, sifting litter, 1400-1800 m asl, Griswold C., Scharff N., Ubick D. (CAS).

\section{Description}

\section{Male}

\section{From Mazumbai (CAS)}

Total Length. 2.47. Carapace length 1.10, width 0.88, yellow brown, somewhat iridescent, with a thin border and grey mottling on sides (Figs 2E, 9A). Fovea brown, pronounced, length 0.13 , anterior end 0.66 from front end of carapace. MOQ length 0.13 , anterior width 0.11 , posterior width 0.16 , AER width 0.24, PER width 0.32. All eyes subcircular, LE equal in size, their diameter more than twice the diameter of ME, which are also equal in size. AME separated by less than their diameter, almost touching ALE. PME separated by 2.5 times their diameter, 1.5 diameters from PLE (Fig. 9A). Both eye rows recurved from above. Clypeus vertical, equal to diameter of AME. Chilum pale, about the size of the AME group. Chelicerae yellow brown. Labium slightly wider than long, half as long as endites. Sternum yellow, with a thin border, length 0.66 , width 0.58 . PCT weak and pointed.

ABDOMEN. Grey dorsally, covered with long thin hairs, with brown, poorly defined, subtriangular anterior do scutum covering less than $10 \%$ of the do surface area. Ventral side of abdomen greyish white, with
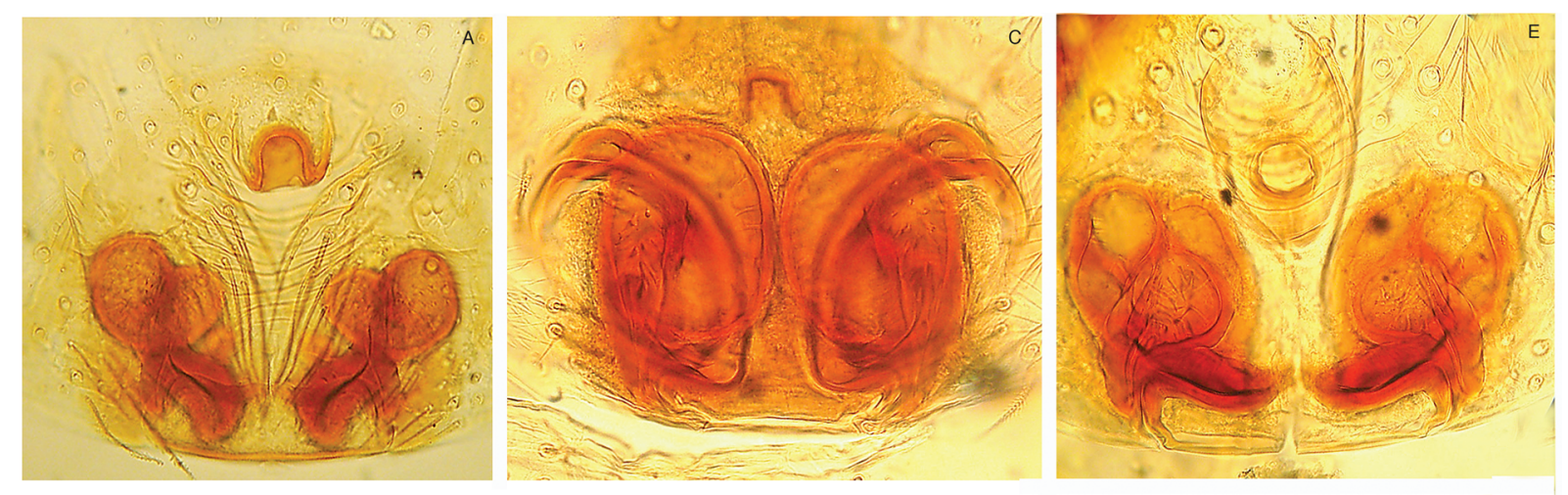
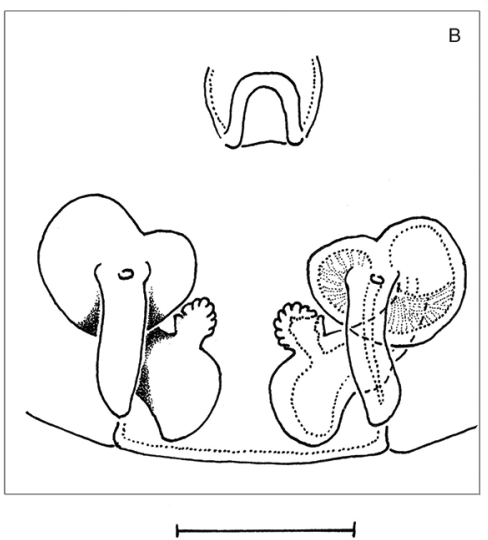

Cteniogaster toxarchus

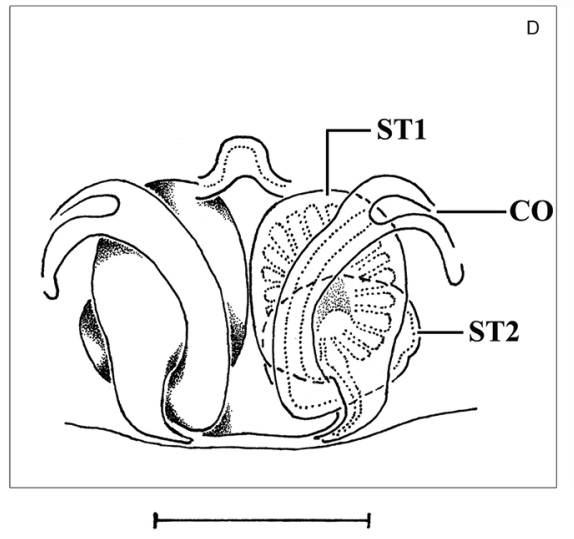

Cteniogaster hexomma

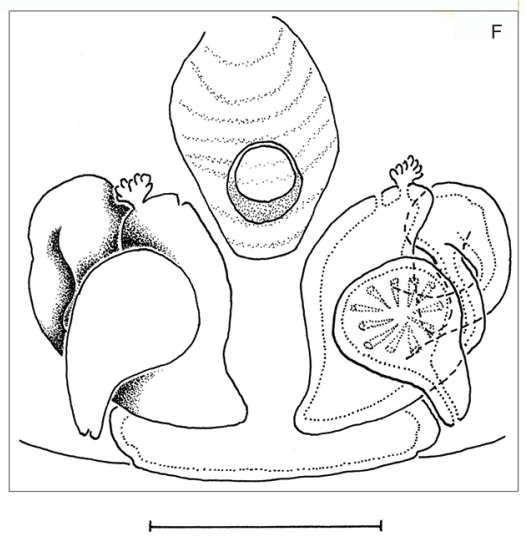

Cteniogaster lampropus

Fig. 4. Female genitalia, ventral view. A, C, E: cleared in methyl salicylate. A-B. Cteniogaster toxarchus gen. et sp. nov. C-D. Cteniogaster hexomma sp. nov. E-F. Cteniogaster lampropus sp. nov. Scale bars $=0.1 \mathrm{~mm}$. 
a brown sclerotised elliptic posterior patch carrying long, strong setae (Figs 2F, 9B). ALS separated by their diameter. Legs pale brown, somewhat iridescent. Retrocoxal hymen pronounced, subglobular. Tarsus IV bent. Leg spination as in Appendix 1C.

LEG MEASUREMENTS:

\begin{tabular}{|l|c|c|c|c|c|c|}
\hline & $\mathbf{f e}$ & $\mathbf{p a}$ & $\mathbf{t i}$ & $\mathbf{m t}$ & $\mathbf{t a}$ & Total \\
\hline I & 0.92 & 0.37 & 0.79 & 0.63 & 0.53 & 3.23 \\
\hline II & 0.79 & 0.37 & 0.60 & 0.53 & 0.47 & 2.76 \\
\hline III & 0.71 & 0.26 & 0.53 & 0.60 & 0.47 & 2.58 \\
\hline IV & 0.95 & 0.39 & 0.87 & 0.87 & 0.60 & 3.68 \\
\hline
\end{tabular}

MALE PALP. As illustrated (Figs 9C-D, 11A), with a straight, blunt, subtrapezoidal RTA, a short, pointed, pl apical embolus, a median, fan shaped membranous apical conductor and a simple pointed subtriangular rl apical MA with a pl membranous border (Fig. 11A).

\section{Female (holotype)}

Total LenGth. 2.68. Carapace length 1.16 , width 0.95 , brown, laterally mottled as in male (Fig. $2 \mathrm{G}$ ). Fovea pronounced, length 0.16, anterior end 0.66 from front end of carapace (Fig. 9H). MOQ length 0.11 , anterior width 0.11 , posterior width 0.15 . AER width 0.27 , PER width 0.36 . Relative eye sizes, eye row curvatures and clypeus as in male. Chilum as in male, but wider. Sternum brown, with a thin border, length 0.71 , width 0.66. PCT as in male.

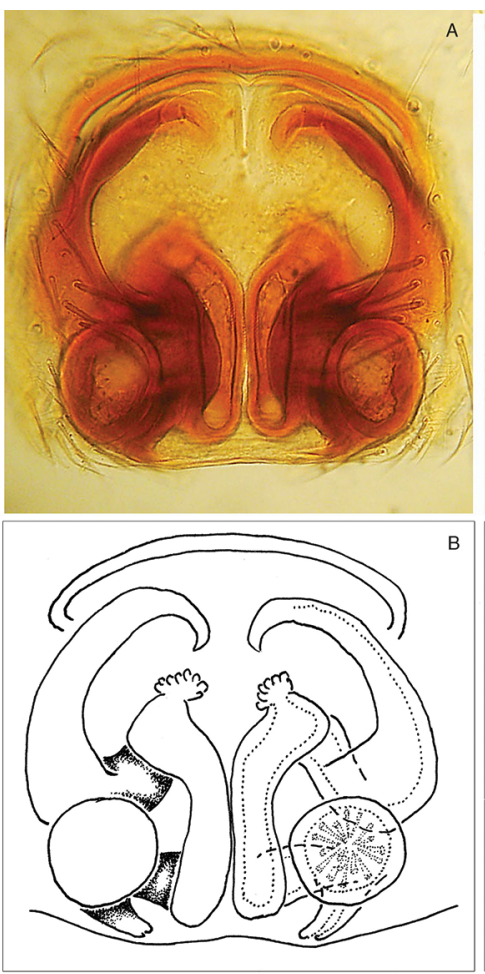

$\vdash$

Cteniogaster conviva
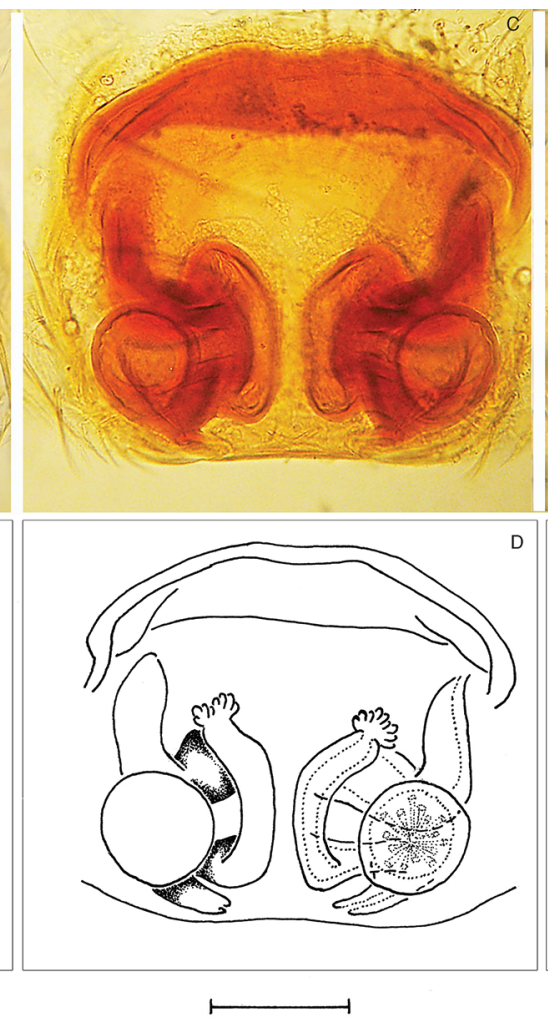

Cteniogaster sangarawe

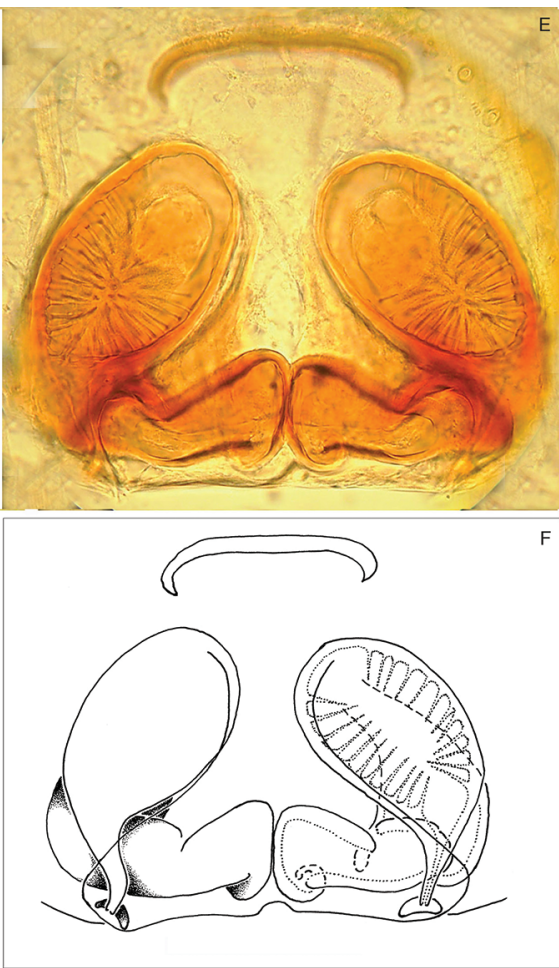

Cteniogaster taxorchis

Fig. 5. Female genitalia, dorsal view. A, C, E: cleared in methyl salicylate. A-B. Cteniogaster conviva sp. nov. C-D. Cteniogaster sangarawe sp. nov. E-F. Cteniogaster taxorchis sp. nov. Scale bars $=0.1 \mathrm{~mm}$. 
Abdomen. Coloured as in male, but without do scutum or ve modified setae. Legs pale brown, somewhat iridescent. Retrocoxal hymen large, oval, subglobular and pearly white. Leg spination as in Appendix 1F.

LEG MEASUFREMENTS:

\begin{tabular}{|l|c|c|c|c|c|c|}
\hline & $\mathbf{f e}$ & $\mathbf{p a}$ & $\mathbf{t i}$ & $\mathbf{m t}$ & $\mathbf{t a}$ & Total \\
\hline I & 1.00 & 0.42 & 0.79 & 0.60 & 0.50 & 3.31 \\
\hline II & 0.89 & 0.34 & 0.68 & 0.53 & 0.47 & 2.92 \\
\hline III & 0.79 & 0.32 & 0.58 & 0.66 & 0.47 & 2.81 \\
\hline IV & 1.08 & 0.39 & 0.89 & 1.03 & 0.63 & 4.02 \\
\hline
\end{tabular}

EPIGYNe. With a small, narrow anterior hood, and two posterior lateral ridges, semitransparent and showing spermatecae in posterior half (Figs 2H, 9E). Vulva with widely separated, kidney-shaped median ST1 with thin internal spikes and posterior ST2 carrying a porous glandular structure (Figs 4AB, 8D-F).

\section{Distribution}

Tanzania, East and West Usambara mountains, 950-1800 m asl.

Cteniogaster hexomma sp. nov. urn:1sid:zoobank.org:act:1893EEE5-2CBC-43AA-9669-4A2A293A89CD

Figs 2I-P, 4C-D, 6, 7, 8G-H, 10A-E, 11B-D, 14; Appendix 1D, G

\section{Diagnosis}

Cteniogaster hexomma sp. nov. differs from all other Cteniogaster gen. nov. species by the consistent absence of PME, by a male palp with a small, straight and pointed RTA and a simple, blunt and scoopshaped rl apical MA, and by the vulva with large, touching, oval ST1 with conspicuous internal spikes and smaller, more dorsally positioned posterior ST2 carrying an anterior porous glandular structure.

\section{Etymology}

The species name is a noun in apposition and refers to the consistent absence of PME in this species, resulting in an array of six eyes.

\section{Type material}

\section{Holotype}

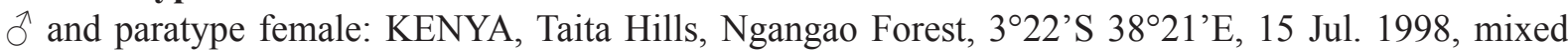
forest, pitfall trap, Rogo L. (MRAC 208888).

\section{Paratypes}

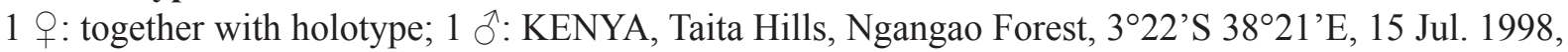
4 Dec. 1999, Winkler extraction of forest litter, VandenSpiegel D. \& Michiels J. (MRAC 209203); 1 O : as previous, 11 Mar. 2004, pitfalls, Spanhove T. \& Chovu M. (MRAC 222071); $2 \hat{\delta}$ : as previous, 11 Mar. 2004 (MRAC 222412); 4 우: as previous, 11 Mar. 2004 (MRAC 222419); 2 우: as previous, 11 Mar. 2004 (MRAC 222426); 1 \%: as previous, 7 Mar. 2004 (MRAC 222431); 1 đ: as previous, 11

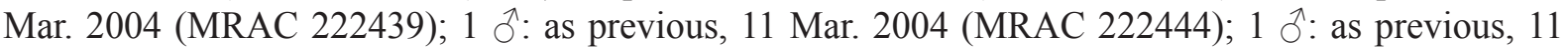

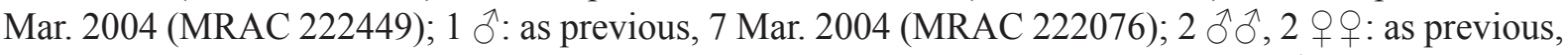
8 Feb. 2004 (MRAC 222084); 1 q: as previous, 8 Feb. 2004 (MRAC 222110); 1 ఫ̃: as previous, 10 Feb. 2004 (MRAC 222086); 1 ㅇ: as previous, 8 Feb. 2004 (MRAC 222088); $2 \hat{\jmath}, 2$ $q \circ$ : as previous, 6 Feb. 2004 (MRAC 222089); 1 ㅇ: as previous, 6 Feb. 2004 (MRAC 222092); 6 Feb. 2004 (MRAC

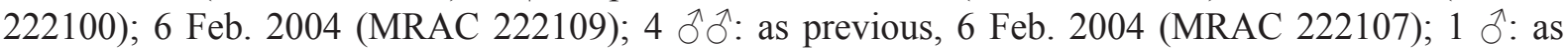


BOSSELAERS J. \& JOCQUÉ R., A new afrotropical genus of Cybaeodinae (Araneae, Liocranidae)
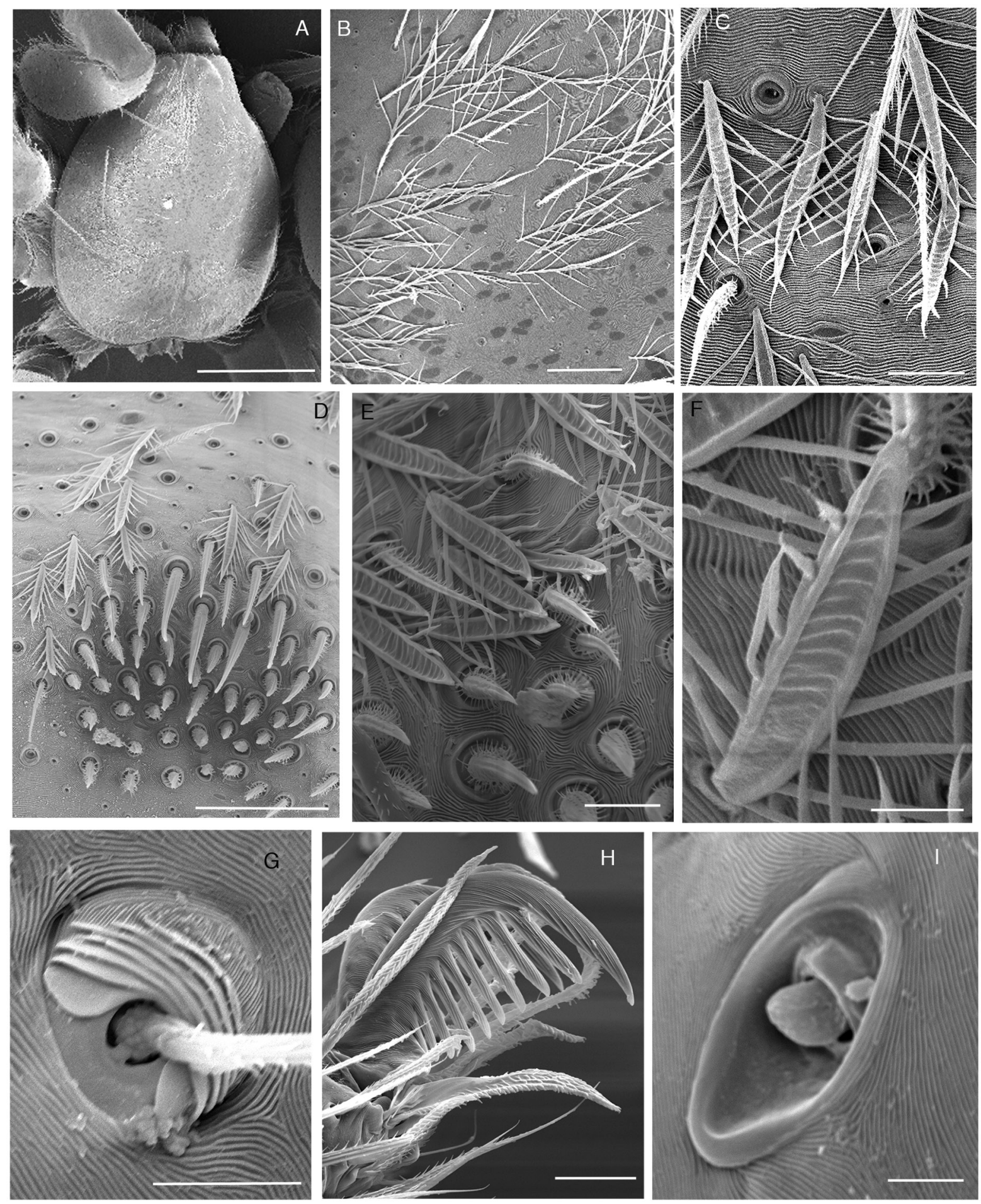

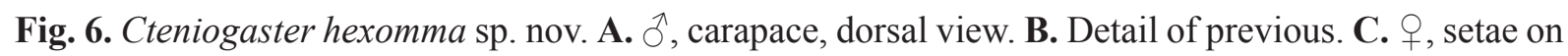
venter of abdomen. D. $\widehat{A}$, group of modified setae on venter of abdomen. E. Detail of previous. F. Detail of seta surrounding patch of modified setae. G. + , trichobothrium on tibia I. H. + , tarsal claws leg I. I. , tarsal organ leg I. Scale bars: A $=0.5 \mathrm{~mm} ; \mathrm{D}=50 \mu \mathrm{m} ; \mathrm{B}, \mathrm{H}=20 \mu \mathrm{m} ; \mathrm{C}, \mathrm{E}=10 \mu \mathrm{m} ; \mathrm{F}, \mathrm{G}, \mathrm{I}=5 \mu \mathrm{m}$. 

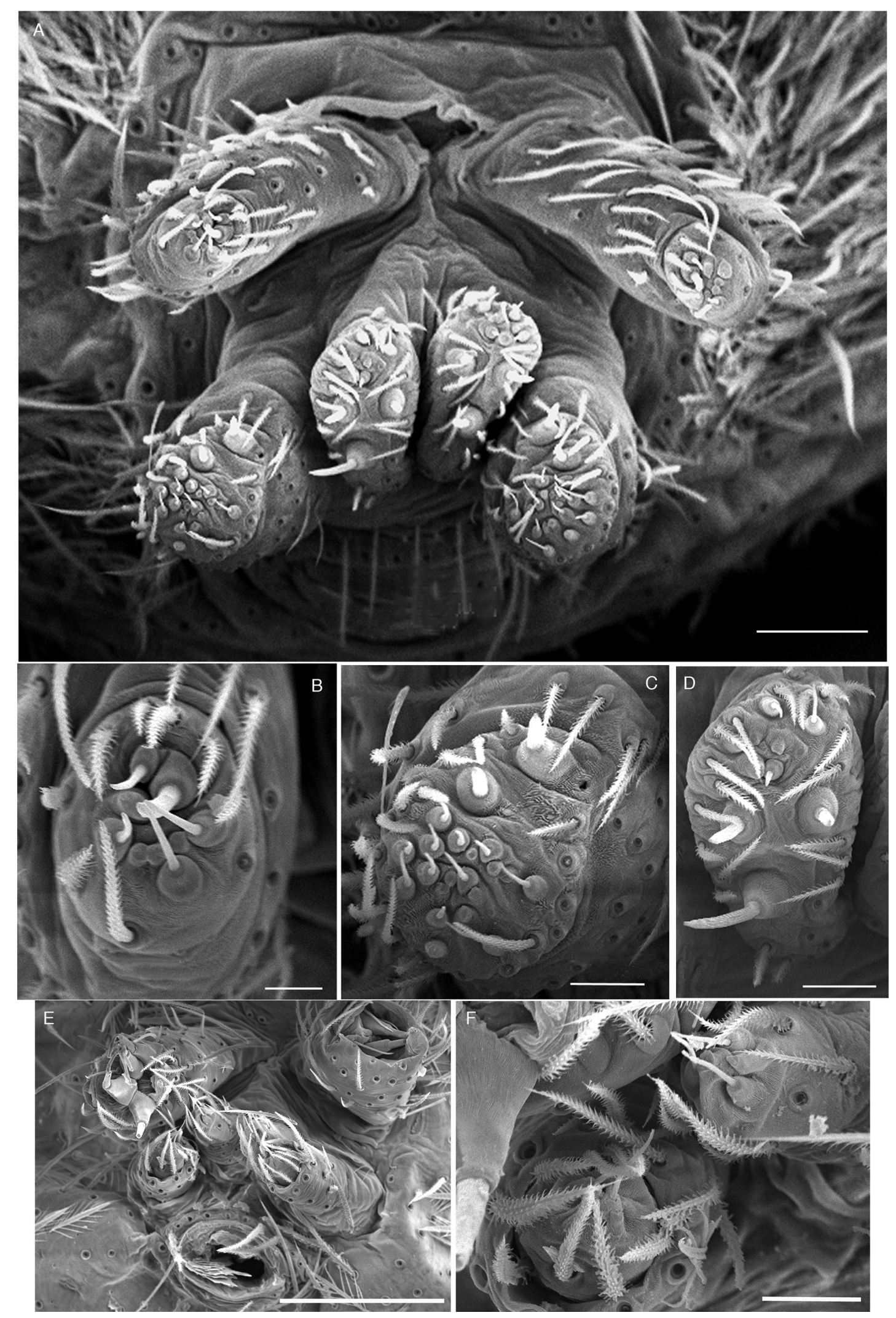

Fig. 7. Cteniogaster hexomma sp. nov. A-D. . A. Apinnerets, posterior view. B. ALS. C. PLS. D. PMS.

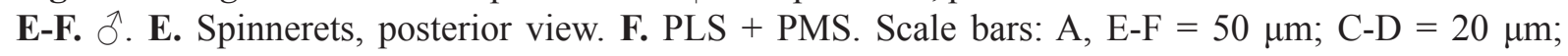
$\mathrm{B}=10 \mu \mathrm{m}$. 
BOSSELAERS J. \& JOCQUÉ R., A new afrotropical genus of Cybaeodinae (Araneae, Liocranidae)

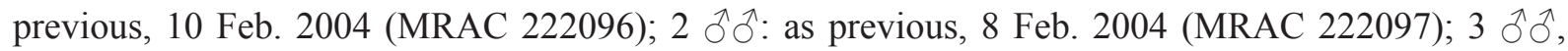

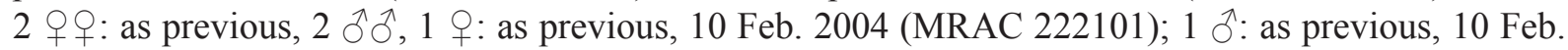

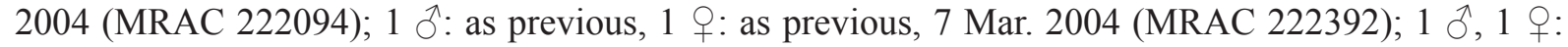
as previous, 10 Apr. 2004 (MRAC 223157); 1 O : as previous, 8 Apr. 2004 (MRAC 223165); 1 के: as previous, 6 Apr. 2004 (MRAC 223230).

\section{Other material examined}

$1 \mathrm{o}^{`}$ : KENYA, Taita Hills, Chawia Forest, 03²9’S 038²0’E, 18-24 Mar. 1998, pitfalls, Rogo Lucy (MRAC 208374); 1 §: as previous, 16 Jul. 1998 (MRAC 208886); 1 q: as previous, 22 Feb. 2004, pitfalls, Spanhove T. \& Chovu M. (MRAC 221973); 3 ỗ: as previous (MRAC 222090); 1 o: as previous (MRAC 222095); 1 §: as previous (MRAC 222004); 1 §, 1 ○: as previous, 23 Feb. 2004, pitfalls (MRAC 222012); 1 O: as previous (MRAC 222085); 1 o: as previous, 24 Feb. 2004 (MRAC 222091); 1 古: as previous, 8 Mar. 2004 (MRAC 222475); 1 o: as previous, 22 Mar. 2004 (MRAC 222784); 1 $\delta$ : as previous (MRAC 222789); 1 $\delta^{\Uparrow}$ : as previous (MRAC 222883); 1 $\delta$ : as previous, 24 Apr. 2004 (MRAC 223253); 1 ○: as previous, 22 Apr. 2004 (MRAC 223271); 1 ○: Taita Hills, Yale Forest, $03^{\circ} 39^{\prime} \mathrm{S} 038^{\circ} 33^{\prime} \mathrm{E}, 6$ Dec. 1999, Winkler extraction of forest litter, VandenSpiegel D. \&

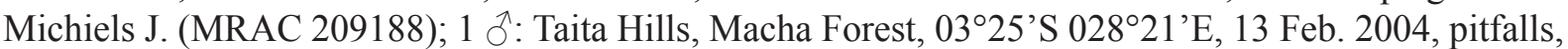
Spanhove T. \& Chovu M. (MRAC 222087); 1 $\delta$ : as previous (MRAC 222093); 2 $\delta$ : Taita Hills, Fururu Forest, 03²6’S 038²0’E, 19 Feb. 2004, pitfalls, Spanhove T. \& Chovu M. (MRAC 222098);

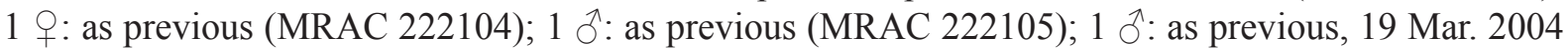
(MRAC 222860); 1 : as previous (MRAC 222892); 1 ¿ : as previous, 19 Apr. 2004 (MRAC 223289);

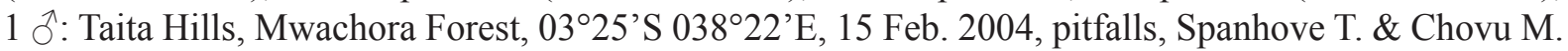
(MRAC 222108); 1 \%: as previous, 15 Mar. 2004, pitfalls, Spanhove T. \& Chovu M. (MRAC 222830);

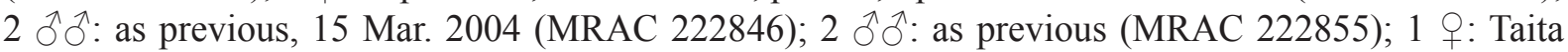
Hills, Wundanyi Forest, $03^{\circ} 24^{\prime}$ 'S $038^{\circ} 22^{\prime}$ E, 17 Feb. 2004, pitfalls, Spanhove T. \& Chovu M. (MRAC 222099); 2 ठ

\section{Description}

\section{Male (holotype)}

Total Length. 2.92. Carapace length 1.34, width 0.97, brown, somewhat iridescent with green and purple hues, unbordered (Fig. 2M-N). Fovea pronounced, length 0.13 , anterior end 0.89 from front end of carapace. AER width 0.21 , PER width 0.29 . AME diameter $1 / 3$ of ALE, separated by their diameter, almost touching ALE, PLE as large as ALE, PME absent (Fig. 10A). Clypeus vertical, equal to 1.5 times the diameter of AME. Chilum about the size of the AME group. Sternum brown, with a thin border, length 0.76 , width 0.63 . PCT brown and pointed.

AвDOMEn. Pale greyish brown dorsally, covered with flattened, iridescent hairs, provided with small, brown, poorly defined anterior do scutum. Ventral side of abdomen pale grey, with a brown sclerotised elliptic posterior patch carrying long, strong setae (Figs 2O-P, 6D-E, 10B). ALS separated by their diameter, PLS with slender conical apical segment, separated by their length. Legs brown, iridescent. Sparse ve terminal preening brushes on mt III and IV. Retrocoxal hymen oval, white, subglobular. Tarsus IV bent. Leg spination as in Appendix 1D.

LEG MEASUREMENTS:

\begin{tabular}{|l|c|c|c|c|c|c|}
\hline & $\mathbf{f e}$ & $\mathbf{p a}$ & $\mathbf{t i}$ & $\mathbf{m t}$ & $\mathbf{t a}$ & Total \\
\hline I & 0.79 & 0.34 & 0.63 & 0.47 & 0.42 & 2.66 \\
\hline II & 0.68 & 0.34 & 0.55 & 0.45 & 0.42 & 2.45 \\
\hline III & 0.66 & 0.29 & 0.45 & 0.50 & 0.47 & 2.37 \\
\hline IV & 0.87 & 0.34 & 0.74 & 0.79 & 0.58 & 3.31 \\
\hline
\end{tabular}


MaLe PalP. As illustrated (Figs 10C-D, 11B), with a small, straight and pointed RTA, a short, pointed, pl apical embolus, a median, fan shaped membranous apical conductor and a simple, blunt and scoopshaped rl apical MA (Fig. 11B).

\section{Female}

Total Length. 2.97. Carapace length 1.10, width 0.88, coloured as in male (Fig. 2I-J). Fovea pronounced, length 0.18 , anterior end 0.76 from front end of carapace. AER width 0.23 , PER width 0.31 . Relative eye sizes, AER curvature and clypeus as in male. PME absent. Chilum a small, yellow brown equilateral triangle beneath AME group. Sternum yellow brown, with a thin border, length 0.68, width 0.63 (Fig. 2L). PCT pointed but weakly sclerotised.

AbDomen. Coloured as in male, but without do scutum or ve modified setae. ALS separated by their diameter, PLS with hemispherical apical segment, separated by their length. Legs yellow brown, iridescent. Retrocoxal hymen as in male. Trochanter notch more prominent in legs III and IV. Preening brushes as in male, tarsus IV slightly bent. Leg spination as in Appendix 1G.

LEG MEASUREMENTS:

\begin{tabular}{|l|c|c|c|c|c|c|}
\hline & $\mathbf{f e}$ & $\mathbf{p a}$ & $\mathbf{t i}$ & $\mathbf{m t}$ & $\mathbf{t a}$ & Total \\
\hline I & 0.76 & 0.34 & 0.60 & 0.39 & 0.42 & 2.52 \\
\hline II & 0.71 & 0.29 & 0.53 & 0.42 & 0.39 & 2.34 \\
\hline III & 0.60 & 0.29 & 0.42 & 0.50 & 0.45 & 2.26 \\
\hline IV & 0.82 & 0.32 & 0.71 & 0.79 & 0.60 & 3.23 \\
\hline
\end{tabular}

EPIGYNE. With a small, narrow anterior hood, semitransparent and showing the copulatory ducts and the large, oval ST1 (Figs 2K, 10E). Vulva with large, touching, oval ST1 with conspicuous internal spikes and smaller, more dorsally positioned posterior ST2 carrying an anterior porous glandular structure (Figs 4C-D, 8G-H).

\section{Distribution}

Kenya, Taita hills, 1600-1900 m asl.

Cteniogaster conviva sp. nov. urn:1sid:zoobank.org:act:EB0512BB-1926-4CC2-88D8-A541A5EDC2F7

Figs 2A-D, 5A-B, 8A-C, 12A-E, 14; Appendix 1E, H

\section{Diagnosis}

Cteniogaster conviva sp. nov. differs from all other Cteniogaster gen. nov. species by the large male palp with a sharply pointed, hook-shaped RTA, a large, coiled and sclerotised conductor, and a cymbium with a rl basal bulge; and by the epigyne with a thin, broad anterior hood.

\section{Etymology}

The species name is a noun in apposition and is derived from the Latin conviva, table companion, referring to the fact that this species shares the same habitat with $C$. toxarchus sp. nov. in the West Usambara mountains.

\section{Type material}

\section{Holotype}

$0^{\top}$ : TANZANIA, Tanga, W. Usambara Mts., Mazumbai Forest, 449’'S 38³0’'E, 11-20 Nov. 1998, 1400$1800 \mathrm{~m}$ asl, sifting litter, Griswold C., Scharff N. \& Ubick D. (ZMUC). 
BOSSELAERS J. \& JOCQUÉ R., A new afrotropical genus of Cybaeodinae (Araneae, Liocranidae)

\section{Paratype}

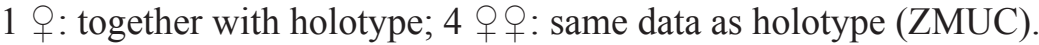

\section{Description}

\section{Male (holotype)}

Total Length. 2.24. Carapace length 1.05, width 0.82, unicoloured yellow, unbordered (Fig. 2A). Fovea brown, pronounced, length 0.13 , anterior end 0.59 from front end of carapace. MOQ length 0.09 , anterior width 0.05 , posterior width 0.08 . AER width 0.19 , PER width 0.24. AME small, diameter $1 / 3$ of diameter ALE. PLE slightly smaller than ALE. All eyes touching, except for PME which are isolated, strongly reduced and flattened, diameter 1/3 of AME diameter (Fig. 12E). AER recurved and PER straight from above. Clypeus vertical, slightly higher than diameter of AME. Chilum indistinct and very small. Sternum yellow, without a distinct border, length 0.60 , width 0.53 . PCT pale yellow.

AвDOMEn. Pale greyish brown, with a weak anterior do scutum covering $10 \%$ of the do surface area and a ve posterior patch of strong setae as is typical for the genus. ALS separated by $1 / 3$ of their diameter. Legs yellow. Retrocoxal hymen pale and weak. Trochanter notch present, more prominent on legs III and IV. Tarsus IV slightly bent. Leg spination as in Appendix 1E.

LEG MEASUREMENTS:

\begin{tabular}{|l|c|c|c|c|c|c|}
\hline & $\mathbf{f e}$ & $\mathbf{p a}$ & $\mathbf{t i}$ & $\mathbf{m t}$ & $\mathbf{t a}$ & Total \\
\hline I & 0.79 & 0.32 & 0.60 & 0.42 & 0.47 & 2.60 \\
\hline II & 0.66 & 0.32 & 0.53 & 0.39 & 0.42 & 2.31 \\
\hline III & 0.55 & 0.26 & 0.39 & 0.42 & 0.39 & 2.03 \\
\hline IV & 0.79 & 0.37 & 0.60 & 0.71 & 0.55 & 3.02 \\
\hline
\end{tabular}

MALE PALP. As illustrated (Fig. 12A-B), with a hook-shaped, sharply pointed RTA, a short apical embolus, a simple subtriangular MA and a complex, coiled, sclerotised conductor. The cymbium has a thickened basal rl bulge (Fig. 12B).

\section{Female}

Total Length. 2.58. Carapace length 1.08, width 0.82, unicoloured yellow, unbordered (Fig. 2A). Fovea brown, pronounced, length 0.13 , anterior end 0.71 from front end of carapace. MOQ length 0.09 , anterior width 0.06 , posterior width 0.10. AER width 0.22 , PER width 0.26 . AME small, diameter $1 / 3$ of ALE diameter. PLE diameter 2/3 of ALE. All eyes touching, except for PME. PME absent or strongly reduced. Eye row curvatures as in male. Clypeus vertical, slightly higher than diameter of AME. Chilum a small, yellow brown equilateral triangle, situated beneath AME. Sternum yellow, with a weak border, length 0.66 , width 0.59 . PCT pale yellow.

ABDomen. Pale greyish brown, without do scutum or ve modified setae. Legs yellow. Retrocoxal hymen as in male. Trochanter notch as in male. Sparse ve preening brushes on mt III and IV. Tarsus IV slightly bent. Leg spination as in Appendix $1 \mathrm{H}$.

LEG MEASUREMENTS:

\begin{tabular}{|l|c|c|c|c|c|c|}
\hline & $\mathbf{f e}$ & $\mathbf{p a}$ & $\mathbf{t i}$ & $\mathbf{m t}$ & $\mathbf{t a}$ & Total \\
\hline I & 0.74 & 0.34 & 0.58 & 0.42 & 0.42 & 2.50 \\
\hline II & 0.66 & 0.26 & 0.50 & 0.39 & 0.39 & 2.21 \\
\hline III & 0.55 & 0.24 & 0.37 & 0.47 & 0.47 & 2.10 \\
\hline IV & 0.87 & 0.34 & 0.66 & 0.71 & 0.55 & 3.13 \\
\hline
\end{tabular}



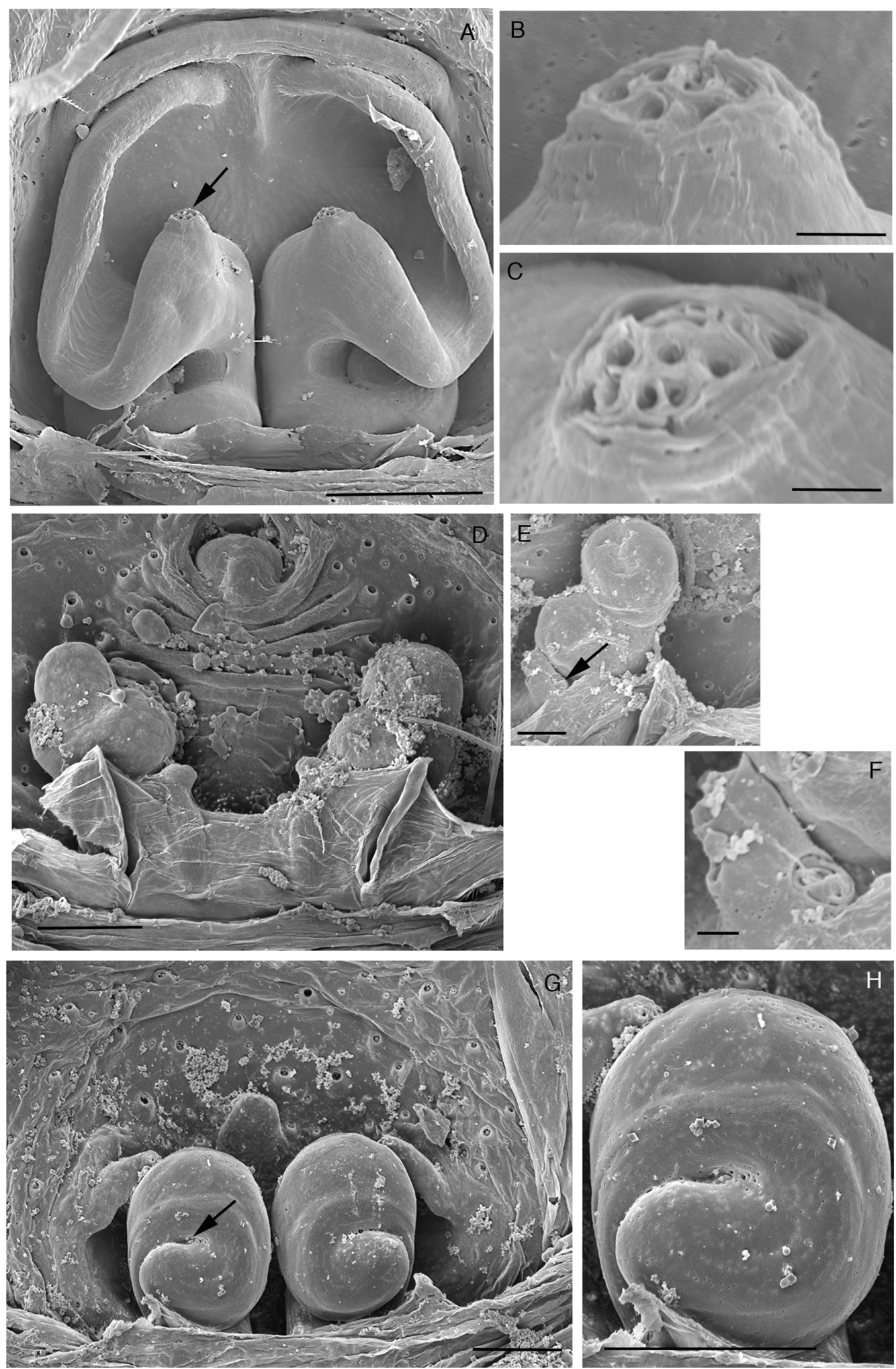

Fig. 8. SEM pictures of female genitalia. A-C. Cteniogaster conviva sp. nov. A. Dorsal view, arrow indicates perforations. B. Detail of previous. C. Idem, seen at different angle. D-F. Cteniogaster toxarchus gen. et sp. nov. D. Dorsal view. E. Detail of other specimen, arrow indicates perforations. F. Detail of previous. G-H. Cteniogaster hexomma sp. nov. G. Dorsal view, arrow indicates peforations. H. Detail of previous. Scale bars $=\mathrm{A}, \mathrm{D}, \mathrm{G}-\mathrm{H}=50 \mu \mathrm{m} ; \mathrm{B}, \mathrm{C}=10 \mu \mathrm{m} ; \mathrm{E}=20 \mu \mathrm{m} ; \mathrm{F}=5 \mu \mathrm{m}$. 
BOSSELAERS J. \& JOCQUÉ R., A new afrotropical genus of Cybaeodinae (Araneae, Liocranidae)

EPIGYNE. With thin, wide anterior hood, partly transparent, showing median ST2 and widely separated posterior globular ST1, slightly variable (Fig. 12C-D). Vulva with widely separated spherical posterior ST1 with internal spikes and median, longitudinally oriented, touching ST2 with an anterior, porous glandular structure (Figs 5A-B, 8A-C).

\section{Distribution}

Tanzania, West Usambara mountains, 1400-1800 m asl.

Cteniogaster sangarawe sp. nov.

urn:1sid:zoobank.org:act:B3900F5F-6616-4A1B-8698-BC1F20748842

Figs 3A-B, 5C-D, 12F-G, 14; Appendix 1K

\section{Diagnosis}

Cteniogaster sangarawe sp. nov. is close to C. conviva sp. nov. but differs from it and from all other Cteniogaster gen. nov. species by the epigyne with a thick and sclerotised, wide anterior hood, and the vulva with median, longitudinally oriented ST2 that are slightly separated from each other.

\section{Etymology}

The species name is a noun in apposition and refers to Sangarawe Forest, the type locality in the East Usambara mountains.

\section{Type material}

\section{Holotype}

: TANZANIA, E. Usambara Mts., Amani, Sangarawe Forest, 5²6.5’S 38³5.7’E, 5-6 Nov. 1995, 990 m asl, sifting litter, Griswold C., Scharff N. \& Ubick D. (ZMUC).

\section{Paratypes}

1, 1 juv: TANZANIA, E. Usambara Mts., Amani, Sangarawe Forest, 55.7’S 38 38’'E, 28 Oct.-9 Nov. 1995, 950 m asl, pitfalls, Griswold C., Scharff N. \& Ubick D. (ZMUC); 2 우 : same data as previous (ZMUC).

\section{Description}

\section{Male}

Unknown.

\section{Female (holotype)}

Total LenGth. 2.24. Carapace length 1.05 , width 0.79 , yellow brown, somewhat iridescent, unbordered (Fig. 3B). Fovea pronounced, length 0.13 , anterior end 0.68 from front end of carapace. MOQ length 0.08 , anterior width 0.07 , posterior width 0.08 . AER width 0.21 , PER width 0.26 . AME small, 1/4-1/3 of ALE diameter. Lateral eyes touching, AME 1.5 times diameter of PLE. PME very small and reduced, diameter $1 / 3$ of AME diameter. Both eye rows straight from above. Clypeus vertical, equal to 1.5 times the diameter of AME. Chilum orange brown, sclerotised. Sternum yellow, length 0.63, width 0.58. PCT very weak, subtriangular.

AвDOMEn. Pale yellowish grey dorsally (Fig. 3B). ALS separated by half their diameter, PLS conical, separated by their length. Legs yellow. Retrocoxal hymen small but pronounced, subglobular, hyaline. Trochanter notch present but not very pronounced. Sparse ve terminal preening brushes on $\mathrm{mt}$ III and IV. Tarsus IV slightly bent. Leg spination as in Appendix $1 \mathrm{~K}$. 
LEG MEASUREMENTS:

\begin{tabular}{|l|c|c|c|c|c|c|}
\hline & $\mathbf{f e}$ & $\mathbf{p a}$ & $\mathbf{t i}$ & $\mathbf{m t}$ & $\mathbf{t a}$ & Total \\
\hline I & 0.76 & 0.39 & 0.55 & 0.39 & 0.42 & 2.52 \\
\hline II & 0.68 & 0.32 & 0.50 & 0.34 & 0.42 & 2.26 \\
\hline III & 0.55 & 0.24 & 0.34 & 0.34 & 0.45 & 1.92 \\
\hline IV & 0.79 & 0.32 & 0.66 & 0.68 & 0.60 & 3.05 \\
\hline
\end{tabular}

Epigyne. with thick and sclerotised, wide anterior hood, partly transparent, showing median ST2 and widely separated posterior globular ST1, slightly variable (Fig. 12F-G). Vulva with widely separated spherical posterior ST1 with internal spikes and median, longitudinally oriented and slightly separated ST2 with an anterior, porous glandular structure (Fig. 5C-D).

\section{Distribution}

Tanzania, East Usambara mountains, 950-990 m asl.

Cteniogaster lampropus sp. nov. urn:1sid:zoobank.org:act:54BD025D-0757-4750-ACEB-403E5B47E984

Figs 3H-J, 4E-F, 12L, 14; Appendix 1I

\section{Diagnosis}

Cteniogaster lampropus sp. nov. differs from all other Cteniogaster gen. nov. species by the relatively large PME, the strongly iridescent legs and the vulva with widely separated piriform posterior ST1 with internal spikes and more median and dorsally situated, broad, longitudinally oriented ST2.

\section{Etymology}

The species name is a noun in apposition and is derived from the Greek $\lambda \alpha \mu \pi \rho \circ \zeta$, shining, and $\pi$ ov $\varsigma$, leg, referring to the strongly iridescent legs.

\section{Type material}

\section{Holotype}

O: TANZANIA, Tanga, W. Usambara Mts., Mazumbai Forest, 449’'S 38²9.5’E, 12-20 Nov. 1995, 1800-1900 m asl, Griswold C., Scharff N. \& Ubick D. (ZMUC).

\section{Description}

\section{Male}

Unknown.

\section{Female (holotype)}

Total Length. 2.42. Carapace length 1.18, width 0.97, brown, with grey radiating striae and dark grey border (Fig. 3H-I). Fovea pronounced, length 0.09 , anterior end 0.83 from front end of carapace. MOQ length 0.13 , anterior width 0.11 , posterior width 0.16 . AER width 0.29 , PER width 0.37 . AME 0.4 of ALE diameter. PLE 0.8 of ALE diameter, separated from them by PLE diameter. PME circular, not reduced, 2/3 of AME diameter. Both eye rows recurved from above (Fig. $3 \mathrm{H})$. Clypeus vertical, equal to AME diameter. Chilum brown, small. Sternum yellow with a darker brown border (Fig. 3J), length 0.74 , width 0.68. PCT pale, subtriangular.

ABDOMEN. Dark greenish grey dorsally, covered with greenish iridescent silky hairs. Ventral side of abdomen creamy brown, darker around spinnerets (Fig. 3J). ALS separated by half their diameter, PLS short and stout, subcylindrical, separated by 1.5 times their diameter. Legs orange, femora olive grey 
BOSSELAERS J. \& JOCQUÉ R., A new afrotropical genus of Cybaeodinae (Araneae, Liocranidae)

(Fig. 3H), iridescent with a green and blue lustre. Retrocoxal hymen subglobular, hyaline. Trochanter notch more pronounced in legs III and IV. Sparse ve terminal preening brushes on mt III and IV. Tarsus IV slightly bent. Leg spination as in Appendix 1I.

LEG MEASUREMENTS:

\begin{tabular}{|l|c|c|c|c|c|c|}
\hline & $\mathbf{f e}$ & $\mathbf{p a}$ & $\mathbf{t i}$ & $\mathbf{m t}$ & $\mathbf{t a}$ & Total \\
\hline I & 0.85 & 0.34 & 0.76 & 0.53 & 0.53 & 3.01 \\
\hline II & 0.76 & 0.34 & 0.63 & 0.53 & 0.50 & 2.76 \\
\hline III & 0.76 & 0.29 & 0.53 & 0.58 & 0.50 & 2.66 \\
\hline IV & 1.03 & 0.39 & 0.84 & 0.92 & 0.63 & 3.81 \\
\hline
\end{tabular}

EPIGYNE. With a narrow anterior hood-like structure, partly transparent, showing superimposed lateral ST1 and ST2 (Fig. 12L). Vulva with widely separated piriform posterior ST1 with internal spikes and more median and dorsally situated, broad, longitudinally oriented ST2 with an anterior, porous glandular structure (Fig. 4E-F).

\section{Distribution}

Tanzania, West Usambara mountains, 1800-1900 m asl.

Cteniogaster taxorchis sp. nov. urn:Isid:zoobank.org:act:DBF6AC89-62A0-4233-A459-D8DB204E7D0E

Figs 3C, G, 5E-F, 12K, 14; Appendix 1K

\section{Diagnosis}

Cteniogaster taxorchis sp. nov. differs from all other Cteniogaster gen. nov. species by the epigyne with a relatively broad, thin, arc-shaped anterior hood, and the vulva with lateral, diagonally oriented anterior ST1 and posterior, transverse ST2 touching in the middle.

\section{Etymology}

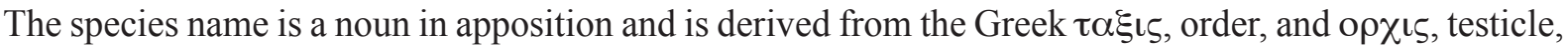
referring to the orderly symmetrical arrangement of the large, testicle-shaped ST1.

\section{Type material}

\section{Holotype}

O: TANZANIA, E. Usambara Mts., Kwamkoro Forest Reserve, 5¹0.9'S 38³5.8’'E, 6 Nov. 1995, $950 \mathrm{~m}$ asl, Griswold C., Scharff N. \& Ubick D. (ZMUC).

\section{Description}

\section{Male \\ Unknown.}

\section{Female (holotype)}

Total Length. 2.21. Carapace length 1.00, width 0.74, unicoloured pale yellow, unbordered (Fig. 3C). Fovea pronounced, length 0.09 , anterior end 0.63 from front end of carapace. MOQ length 0.06 , anterior width 0.06 , posterior width 0.08 . AER width 0.18 , PER width 0.22 . AME $1 / 4$, PME $1 / 3$ and PLE 0.7 of ALE diameter. PME flattened and reduced. Both eye rows recurved from above. Clypeus vertical, equal to twice AME diameter. Chilum indistinct. Sternum pale yellowish white with a yellow border (Fig. 3G), length 0.58 , width 0.53 . PCT indistinct. 
AвDomen. Pale greyish white dorsally. Spinnerets as for the genus in general, but shorter. Legs yellowish white (Fig. 3G). Retrocoxal hymen small, oval, pale. Trochanter notch more pronounced in legs III and IV. Sparse ve terminal preening brushes on mt III and IV. Tarsus IV clearly bent. Leg spination as in Appendix 1K.

LEG MEASUREMENTS:

\begin{tabular}{|l|c|c|c|c|c|c|}
\hline & $\mathbf{f e}$ & $\mathbf{p a}$ & $\mathbf{t i}$ & $\mathbf{m t}$ & $\mathbf{t a}$ & Total \\
\hline I & 0.74 & 0.34 & 0.58 & 0.42 & 0.42 & 2.50 \\
\hline II & 0.63 & 0.26 & 0.45 & 0.37 & 0.39 & 2.10 \\
\hline III & 0.53 & 0.24 & 0.37 & 0.42 & 0.39 & 1.95 \\
\hline IV & 0.79 & 0.28 & 0.61 & 0.66 & 0.55 & 2.89 \\
\hline
\end{tabular}

Epigyne with relatively broad, thin, arc-shaped anterior hood, partly transparent, showing large oval anterior ST1 and transverse posterior ST2 (Fig. 12K). Vulva with lateral, diagonally oriented anterior ST1 with conspicuous internal spikes and posterior, transverse ST2 touching in the middle (Fig. 5E-F).

\section{Distribution}

Tanzania, East Usambara mountains, Kwamkoro Forest, $950 \mathrm{~m}$ asl.

Cteniogaster nana sp. nov. urn:Isid:zoobank.org:act:B0321C9D-7120-407A-BAAF-BCF85186FDA0

Figs 3D-F, 12H-J, 14; Appendix 1B

\section{Diagnosis}

Cteniogaster nana sp. nov. differs from all other Cteniogaster gen. nov. species by its small size and by the male palp with a blunt, dorsally bent RTA, a relatively large and broad prolaterally inserted embolus, a small sclerotised apical conductor and a small and subtriangular retrolaterally inserted MA.

\section{Etymology}

The species name is derived from the Latin nanus, dwarf, and refers to the small size of the present species.

\section{Type material}

Holotype

ơ: TANZANIA, E. Usambara Mts., Amani, 5⒌7’S 38³8’'E, 28 Oct.-9 Nov. 1995, 950 m asl, pitfalls, Griswold C., Scharff N. \& Ubick D. (CAS)

\section{Paratype}

1 to: together with holotype.

\section{Description}

\section{Male (holotype)}

Total Length. 1.74. Carapace length 0.74 , width 0.58 , unicoloured pale yellow, unbordered (Fig. 3C). Fovea pronounced, length 0.08 , anterior end 0.53 from front end of carapace. MOQ length (when eight eyes present) 0.05 , anterior width 0.02 , posterior width 0.06 . AER width 0.11 , PER width 0.15 . Median eyes with a strong tendency towards reduction, one specimen with reduced median eyes, the other having only four eyes (Fig. 12J). AME very small (1/6 of ALE diameter) or absent, PME very pale and strongly reduced ( $1 / 8$ of ALE diameter) or absent, PLE $2 / 3$ of ALE diameter. Both eye rows (if ME are 
present) straight from above. Clypeus vertical, equal to 1/3 of ALE diameter. Chilum indistinct. Sternum yellow, unbordered (Fig. 3D), length 0.47 , width 0.39. PCT indistinct.

AbDomen. Pale cream dorsally (Fig. 3F) with traces of a weak, diffuse anterior do scutum and ventrally with an oblong, sclerotised patch carrying strong modified setae in posterior half (Fig. 3 E). Spinnerets as for the genus in general. Legs pale yellow (Fig. 3D, F). Retrocoxal hymen pronounced, oval and white. Trochanter notch indistinct in legs I and II, pronounced in legs III and IV. Very sparse ve terminal preening brushes on mt III and IV. Tarsus IV slightly bent. Leg spination as in Appendix 1B.

LEG MEASUREMENTS:

\begin{tabular}{|l|c|c|c|c|c|c|}
\hline & $\mathbf{f e}$ & $\mathbf{p a}$ & $\mathbf{t i}$ & $\mathbf{m t}$ & $\mathbf{t a}$ & Total \\
\hline I & 0.53 & 0.24 & 0.39 & 0.29 & 0.28 & 1.72 \\
\hline II & 0.45 & 0.22 & 0.37 & 0.28 & 0.28 & 1.59 \\
\hline III & 0.42 & 0.14 & 0.29 & 0.32 & 0.29 & 1.46 \\
\hline IV & 0.55 & 0.21 & 0.47 & 0.50 & 0.39 & 2.13 \\
\hline
\end{tabular}

MALE PALP. With a blunt, dorsally bent RTA, a relatively large and broad prolaterally inserted pointed embolus, a small sclerotised apical conductor, and a pointed, small and subtriangular retrolaterally inserted MA.

\section{Female}

Unknown.

\section{Distribution}

Tanzania, East Usambara mountains, $950 \mathrm{~m}$ asl.

\section{Discussion}

\section{Phylogenetic analyses}

The preferred consensus tree for 27 species known from both sexes, with node numbers, state changes for 47 characters and Goloboff fit Bremer support values (as reported in TNT) in italics below branches is illustrated in Fig. 1. Each ambiguity on the tree was optimized in isolation, in order to avoid scoring character states for absent structures, and also because only a combination of ACCTRAN and DELTRAN optimisation can produce the most robust proposal for a supposed homology. Indeed, the use of ACCTRAN only, as is often preferred, does not always maximize parallel loss of complex traits over convergent gains (Agnarsson \& Miller 2008). Of the five ambiguous characters shown on the preferred tree in Fig. 1, DELTRAN optimisation was preferred for characters 19, 38 and 72, ACCTRAN for character 37, and only unambiguous changes are shown for character 22.

Homoplasy in the data matrix which produced the preferred tree is quite acceptable: 22 out of 99 characters are completely free of homoplasy. Sanderson \& Donoghue (1989: 1785, fig. 1) performed a polynomial regression analysis on data from 60 cladistic analyses, and derived the following equation based on them: $\mathrm{ci}=0.90-0.022 \times($ number of taxa $)+0.000213 \times(\text { number of taxa })^{2}$. Applying this equation, 27 taxa would yield a ci value of 0.461 , quite similar to the actual ci value of 0.412 obtained for the consensus tree in the present analysis.

The ingroup (clade 1) is supported on the preferred tree by the absence of apical do spines on fe III and IV (11:0, not shown in Fig. 1, reversed in Cybaeodes marinae Di Franco, 1989 and in clade 14), the presence of a distal spine on the male palpal pl edge [27:1, absent in Scotina palliardii (L. Koch, 

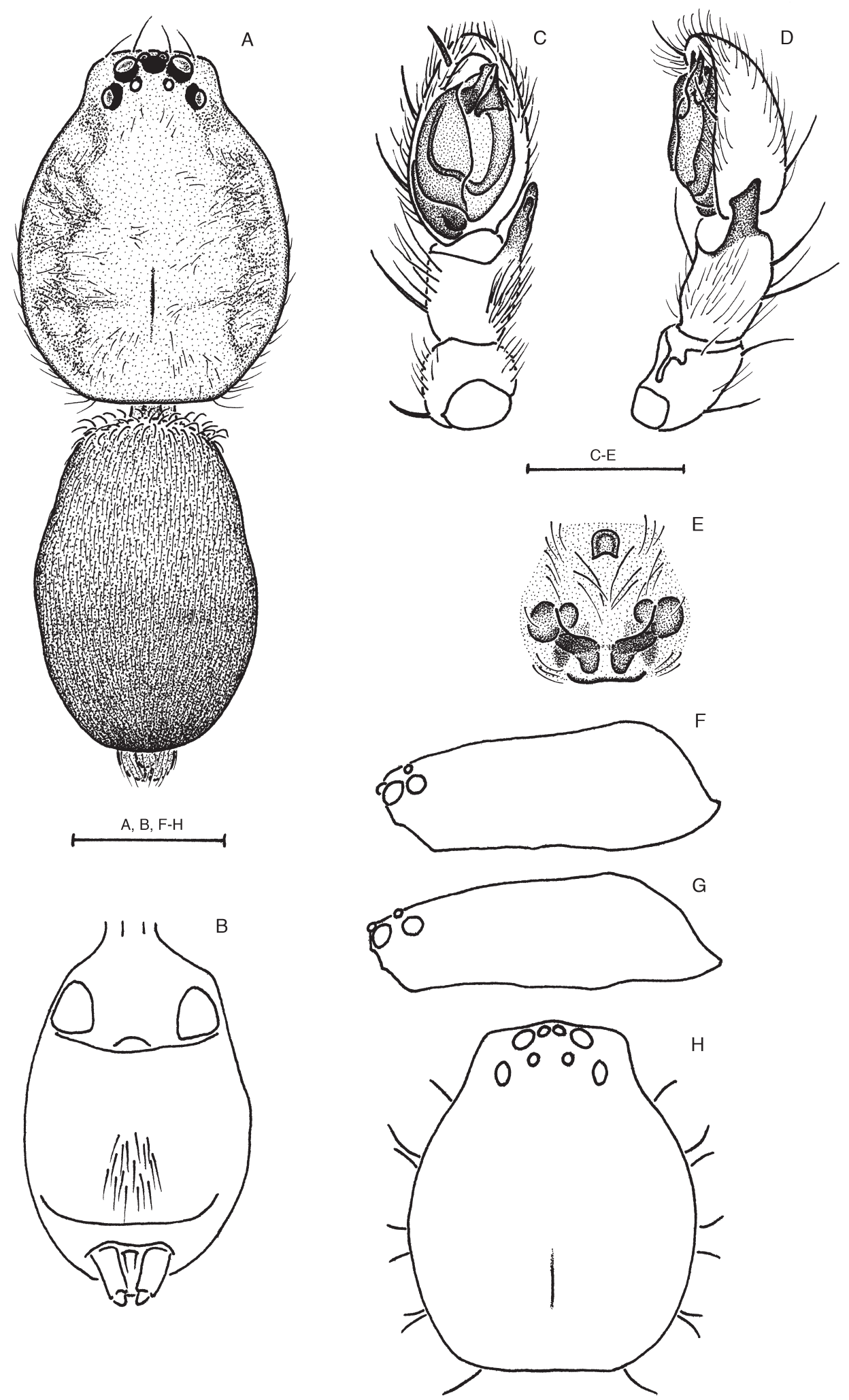

Fig. 9. Cteniogaster toxarchus gen. et sp. nov. A. $\partial^{\jmath}$, habitus, dorsal view. B. Idem, abdomen, ventral view. C. Idem, palp, ventral view. D. Idem, retrolateral view. E. Epigyne, ventral view. F. + , carapace, lateral view. G. ${ }^{\lambda}$, carapace, lateral view. H. + , carapace, dorsal view. Scale bars: A-C, F-H $=0.5 \mathrm{~mm}, \mathrm{C}-\mathrm{E}=0.25 \mathrm{~mm}$. 
1881)], absence of true claw tufts (36:0), the presence of tenent hairs at the tip of tarsi (37:1, reversed in clades 3 and 14, but present in Apostenus), a simple sternal border (42:0, reversed in Toxoniella), two retromarginal cheliceral teeth (43:0, more than two in Toxoniella), a shaggy hair in front of the fang base (44:1, reversed in Toxoniella), a conspicuous serrula on the endites (48:0), a flat carapace (50:1, changed to slanting in clade 20), modified PME (57:1, reversed in clade 14), the MOQ widest posteriorly (58:1), curved strong hairs frontally on abdomen $(60: 1)$, no epigastric sclerite $(62: 0,70: 0)$, laterally compressed female PMS (71:1, changed to slender in clade 4 and to stout and subtriangular in clade 11), absence of a coiled sperm duct (79:0), a membranous conductor (81:1, reversed in Cteniogaster conviva sp. nov. and Agraecina lineata (Simon, 1878), unapplicable in clade 20), a simple conductor (82:0, complex in C. conviva sp. nov., unapplicable in clade 20), MA present (83:1), subtegulum pl (89:0), anterior hood
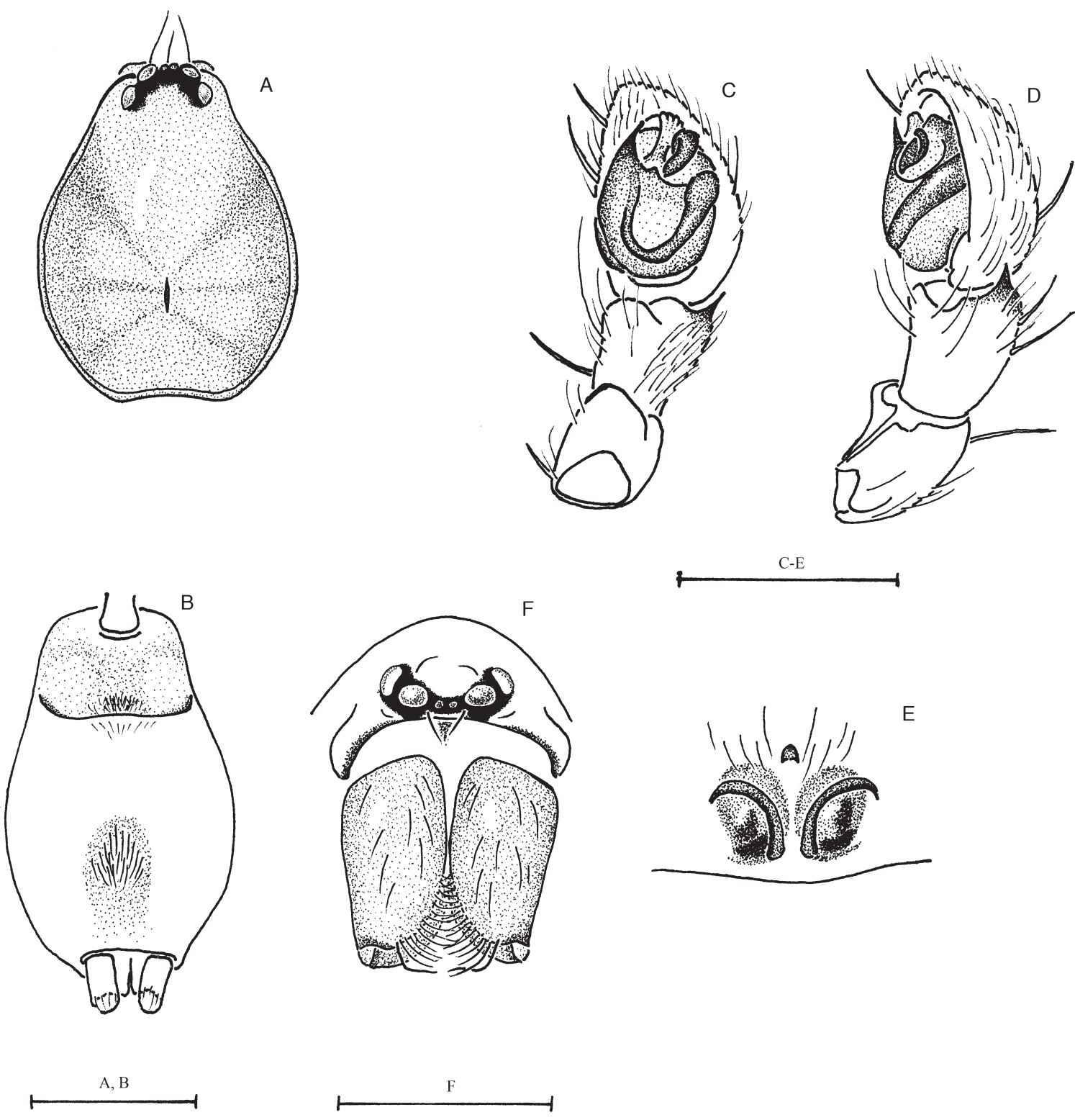

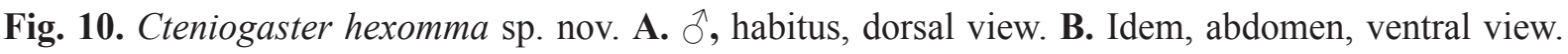
C. Idem, palp, ventral view. D. Idem, retrolateral view. E. Epigyne, ventral view. F. + , carapace, anterior view. Scale bars: A-B, F $=0.5 \mathrm{~mm}, \mathrm{C}-\mathrm{E}=0.25 \mathrm{~mm}$. 
present in epigyne (94:1, reversed in Hesperocranum rothi Ubick \& Platnick, 1991, and in clade 15) and anterior epigynal entrances (97:0). We consider clade 1 to represent the family Liocranidae. It is divided in two sister clades in the present analysis: clade 2 and clade 7 (Fig. 1).

Clade 2 is interpreted as subfamily Liocraninae and is supported on the preferred tree by the presence of large erectile bristles in the ve scopulae of legs I and II (5:1), ventral scopulae on ti I and II (33:1) and a pl terminal lobe on the male palpal ti (91:1). Clade 4 (Mesiotelus Simon, 1897 and Liocranum L. Koch, $1866)$ is further characterised by slender female PMS carrying only a single large spigot (71:0, 72:0, contra Wunderlich 2008: 489).

Clade 7 is interpreted as subfamily Cybaeodinae and is supported on the preferred tree by the presence of a trochanter notch (3:1, reversed in Apostenus), the presence of bent male tarsi (8:1), presence of do spines on ti III (at least in females) and IV (17:1, 18:1), cylindrical ALS in males (65:1, reversed in clade 17), and presence of EPGS in males (69:1, reversed in clade 20). Although present in some Clubionidae as well (see discussion of character 69, above), the presence of EPGS seems to be an interesting apomorphy for the subfamily Cybaeodinae. The character is lost in the genera Apostenus and Scotina, most probably due to their small size. Cybaeodes Simon, 1878 (clade 8) holds a basal position within Cybaeodinae in the present analysis, supported by two rows of more than five large spigots on female PMS (paralleled in Toxoniella) and a small dorsal bristle mat on the male palpal cymbium (76:1). Toxoniella (clade 10) differs from all other members of clade 1 by a number of reversals, being a rebordered sternum (42:1), more than two retromarginal cheliceral teeth (43:1) and absence of a shaggy hair in front of the cheliceral fangs (44:0), but the genus fits within the clade for all other important characters, including the presence of an epigynal anterior hood (94:1). Toxoniella shares with Cybaeodes, Hesperocranum Ubick \& Platnick, 1991 and Sagana Thorell, 1875 the laterally compressed female PMS (71:1), and with Cybaeodes and Sagana a large number of tarsal tenent hairs (38:2). Both characters appear plesiomorphic within the family. Within Cybaeodinae, Toxoniella shares the presence of EPGS (69:1) with most other genera. Toxoniella is herewith transferred to Liocranidae. None of the other genera currently included in Gallieniellidae is reported to possess EPGS in males.

Cteniogaster gen. nov. also fits well within Cybaeodinae, but differs from related genera by the wide patellar indentations (6:1, 7:1, paralleled in Apostenus), the presence of one $\mathrm{mt}$ IV plv and rlv spine (21:1), two pairs of tenent hairs (38:1), and the presence of ve abdominal setae in males (63:1, paralleled in Agroeca brunnea (Blackwall, 1833) and Agrocea cuprea Menge, 1873 (clade 19) and in Apostenus fuscusWestring, 1851). The fact that these male ve abdominal setae occupy a well delimited small oval area (64:1) is an autapomorphy for the genus. Clade 14 is supported by the absence of $\mathrm{mt} \mathrm{III} \mathrm{and} \mathrm{IV} \mathrm{vt}$ spines (23:0, reversed in clade 20) and the presence of circular PME (57:0). Clade 16 is supported by the presence of a subtegular locking lobe (78:1), complemented in clade 17 (the genera Agroeca, Apostenus and Scotina) by a tegular locking lobe (77:1) and conical ALS in males (65:0). The genus Agroeca (clade $18)$ is supported by a bifid MA (84:1, paralleled in Sagana rutilans Thorell, 1875) and a flat, ribbonshaped embolus (87:4). Clade 20, consisting of small, derived Cybaeodinae, is distinguished by plv spines on fe I (9:1), a slanting carapace (50:0), and absence of a conductor (80:0) and of EPGS (69:0). Apostenus is further characterised by the presence of one pair of tenent hairs (38:0), widely separated PME (59:2) and the absence of ST2 (98:0). Its sister genus Scotina features equidistant PE (59:1) and a whip-shaped embolus (87:5).

While some characters $(36: 0,48: 0,58: 1,60: 1,62: 0,70: 0,79: 0,83: 1,89: 0,97: 0)$ are constant throughout Liocranidae, others $(44: 1,57: 1,65: 1,69: 1,94: 1)$ show reversal in some clades.

Reversals and secondary losses of characters are particularly common on the preferred tree in the genus Toxoniella and in clade 20, which groups the genera Apostenus and Scotina. Apart from the three 
reversals mentioned above, Toxoniella has no plv and rlv spines on female ti I (14:0) and has male and female PLS close together (68:0 and 73:0, paralleled in clade 3 and in clade 4, respectively). In clade 20, apart from the already mentioned absence of EPGS (69:0) and a conductor (80:0), the number of mt III plv and rlv spines is reduced (20:0, paralleled in Cteniogaster gen. nov. and Hesperocranum), mt
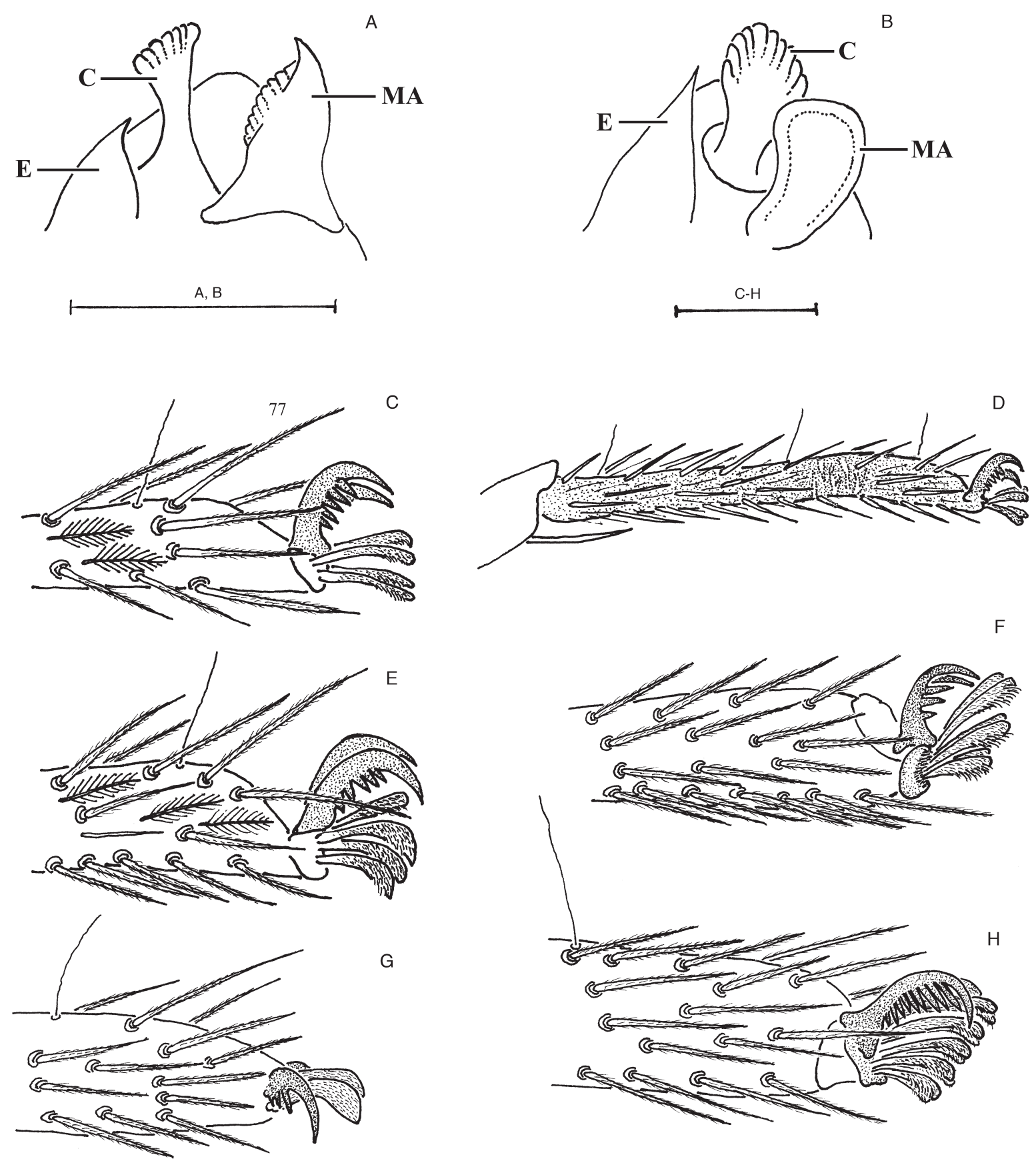

Fig. 11. A. Cteniogaster toxarchus gen. et sp. nov. Tip of male palp, ventral view. B-D. Cteniogaster hexomma sp. nov. B. Tip of male palp, ventral view. C. Tip of male tarsus IV, retrolateral view. D. Bent male tarsus IV. E. Cteniogaster toxarchus gen. et sp. nov., tip of male tarsus IV. F. Apostenus spinimanus (Koch \& Berendt, 1854), tip of male tarsus IV. G. Apostenus fuscus Westring, 1851, tip of male tarsus IV. H. Arabelia pheidoleicomes Bosselaers, 2009, tip of female tarsus IV. Scale bars: D = $200 \mu \mathrm{m}$; A-C, E-G = $100 \mu \mathrm{m}$. Abbreviations: $\mathrm{C}=$ conductor; $\mathrm{E}=$ embolus; $\mathrm{MA}=$ median apophysis. 

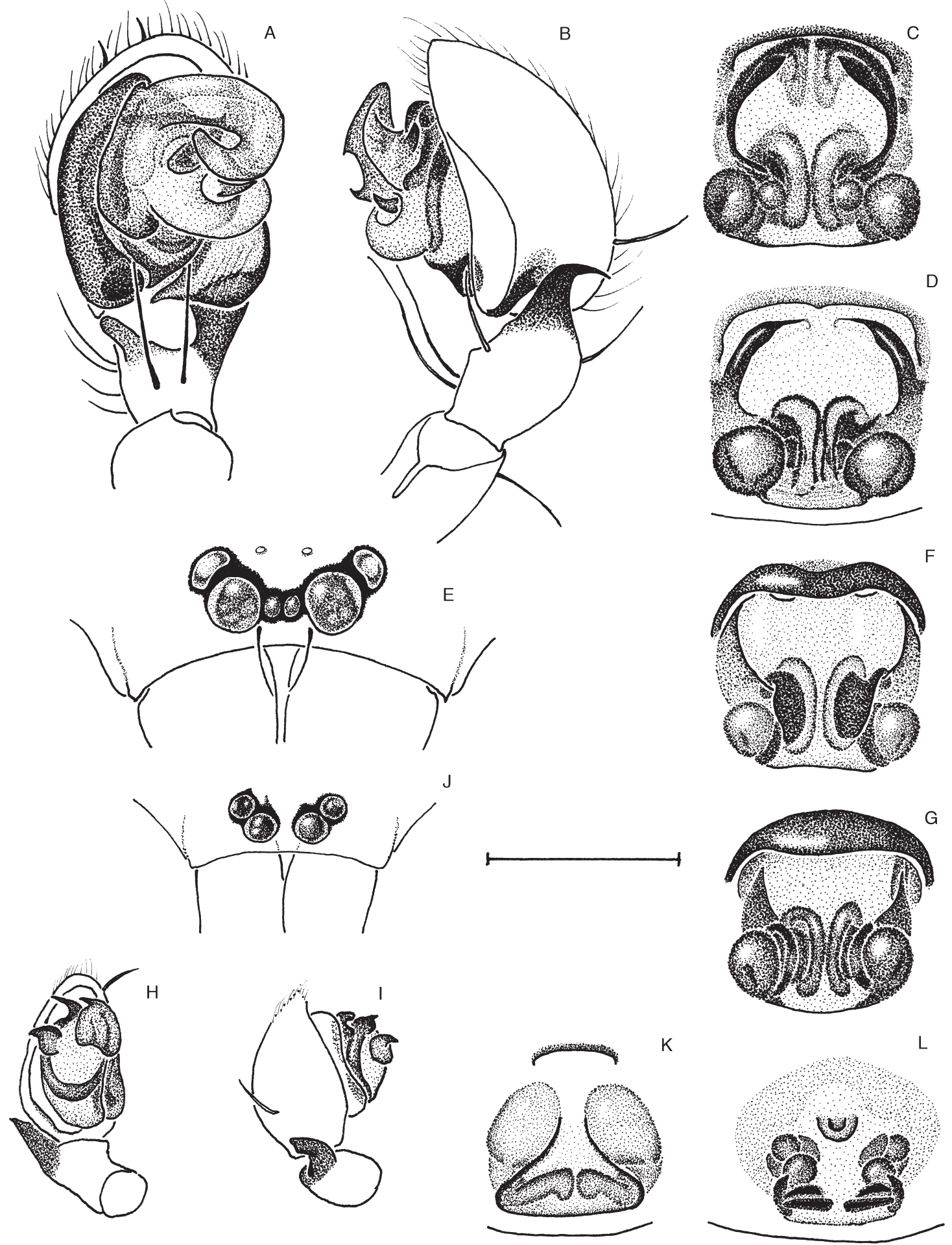

Fig. 12. A-E. Cteniogaster conviva sp. nov. A. $\partial$, palp, ventral view. B. Idem, retrolateral view. C. Epigyne, ventral view. D. Idem, other specimen. E. Eye region of male, anterior view. F-G. Cteniogaster sangarawe sp. nov. F. Epigyne, ventral view. G. Idem, other specimen. H-J. Cteniogaster nana sp. nov. H. ${ }^{\lambda}$, palp, ventral view. I. Idem, retrolateral view. J. Eye region of male, anterior view. K. Cteniogaster taxorchis sp. nov. Epigyne, ventral view. L. Cteniogaster lampropus sp. nov. Epigyne, ventral view. Scale bar $=250 \mu \mathrm{m}$. 
III and IV have ve terminal spines (23:1), feathery hairs are absent (30:0, paralleled in Hesperocranum, Liocranoeca and Agraecina), there is no apical maxillar hair tuft (49:0, paralleled in Cteniogaster gen. nov., Hesperocranum and Liocranoeca), and PME and PLE are close to each other (59:1 or 59:2). Moreover, Apostenus has no trochanter notch (3:0) and no ST2 (98:0). The presence of male modified ve abdominal setae (63:1) appears to have evolved convergently in Cteniogaster gen. nov. and in some Agroeca and Apostenus (Wunderlich 2008: 489). The character was already present in the eocene Apostenus spinimanus. The presence of tenent hairs (37:1) also shows a peculiar distribution on the preferred tree, being restricted to the genera Sagana, Cybaeodes, Toxoniella, Cteniogaster gen. nov., and Apostenus. While Sagana has 9 and Cybaeodes (see Bosselaers 2009) and Toxoniella 5 pairs of tenent hairs, Cteniogaster gen. nov. has only two pairs (Fig. 11C-E) and recent Apostenus only one (Fig. 11G). However, the extinct Apostenus spinimanus, most probably congeneric with recent species, has three pairs (Fig. 11F, contra Wunderlich 2004: 1627). Based on the presence of tenent hairs, modified male ve abdominal setae, an epigynal anterior hood and the other characters listed above for clade 1 (with the exception of a sclerotised conductor for Cteniogaster conviva sp. nov.), the new genus Cteniogaster gen. nov. is placed in Liocranidae, Cybaeodinae.

The present phylogenetic analysis does not produce an unequivocal autapomorphy for Liocranidae. However, a combination of a number of non-homoplasious character changes mentioned above for clade 1 in the discussion of phylogenetic findings, offers significant potential for recognising genera as Liocranidae. No doubt, most of these character states (11:0, 27:1, 42:0, 43:0, 44:1, 48:0, 57:1, 58:1, 60:1, 89:0, 97:0) are not unique to Liocranidae and probably plesiomorphic on a wide scale or only apomorphic to a larger clade in which Liocranidae will prove to be embedded. Nevertheless, we consider a combination of a sizable number of these character states as the best approach available to date towards recognising Liocranidae: the absence of true claw tufts (36:0), the presence of tenent hairs (37:1), the absence of extensive abdominal ve sclerotisation, at least in females (70:0), the presence of a simple, membranous conductor as well as a MA in the male palp $(81: 1,82: 0,83: 1)$ and an epigyne with an anterior hood (94:1). The attribution of the liocranid genera included in the present analysis to subfamilies Liocraninae and Cybaeodinae can be considered robust, as both supraspecific taxa are supported by apomorphies within Liocranidae (5:1, 91:1 and 3:1, 8:1, 17:1, 18:1, 69:1, respectively). The presence of EPGS in males (69:1) is the main character on which the transfer of Toxoniella to Liocranidae, Cybaeodinae is based. The family Gallieniellidae is poorly defined: porrect chelicerae, the only synapomorphy found in Platnick's cladogram (2002: 9), also occur in other araneomorph spider families, such as Clubionidae and Theridiidae Sundevall, 1833. In addition, Haddad et al. (2009: 16) mention small male AME, a recurved PER, conical ALS and a short cymbium tip, but most of these characters reverse somewhere within the Gallieniellidae clade and none is unique to the family. The presence of tenent hairs (37:1), a character found in the majority of Liocranidae, is also encountered in the gallieniellid genera Drassodella Hewitt, 1916 (Warui \& Jocqué 2002: 314) and Austrachelas Lawrence, 1938, although in the latter they are only present on the posterior two pairs of legs, similar to what is described in Raven \& Stumkat (2002) for some Clubionidae. However, no other gallieniellid has cylindrical ALS provided with EPGS in males, and this character, in combination with the above mentioned set of characters commonly encountered in Liocranidae, is judged sufficient to justify the transfer.

As far as the other liocranid genera listed in Platnick (2012) are concerned, some conclusions can be drawn and a few transfers proposed. Argistes Simon, 1897 and Sphingius Thorell, 1890 most probably belong in Liocranidae, given the presence of tenent hairs (Deeleman-Reinhold 2001: figs. 639; personal observation), a simple conductor and a MA. As long as the conspecificity of the male described by Bosmans (2011: 20, figs. 15-16) with females of Arabelia Bosselaers, 2009 is not proved beyond doubt, it seems better to keep the genus as Liocranidae incertae sedis, due to the presence of five pairs of tenent hairs and an anterior epigynal hood, as well as the complete absence of abdominal sclerotisation. Pending 
a generic revision, the same classification is defended for Rhaeboctesis Simon, 1897, given the absence of true claw tufts and abdominal sclerotisation, as well as the presence of a simple conductor and a MA. Andromma Simon, 1893 does not fit well in Liocranidae as defined here, due to the presence of true claw tufts and the absence of a MA and anterior epigynal hood. It is likely to belong in Corinnidae, but since a comprehensive cladistic analysis of a larger number of liocranid and corinnid genera is not yet available, it is best to keep it in Liocranidae as incertae sedis. Paratus Simon, 1898, which lacks a MA (Marusik et al. 2008; Zapata \& Ramírez 2010) was placed in a subfamily of its own by Marusik et al., based, apart from the general character states already mentioned above for clade 1, on an insuffient number of characters (2008: 51), such as absence of a retrocoxal hymen (also absent in Cybaeodes, Liocranoeca, Neoanagraphis and Sagana, see Appendix 3), embolus inserted centrally on tegulum, very simple epigyne and abdomen with guanine spots. It seems best to keep the genus as Liocranidae incertae sedis until a thorough analysis has been performed. Sudharmia Deeleman-Reinhold, 2001, with its almost unsclerotised female abdomen and male palp with pl subtegulum and simple, membranous conductor (but lacking MA) is also considered Liocranidae incertae sedis here. Literature data on Heterochemnis F.O.P. Cambridge, 1900, Laudetia Gertsch, 1941, Liparochrysis Simon, 1909 and Mesobria Simon, 1897,

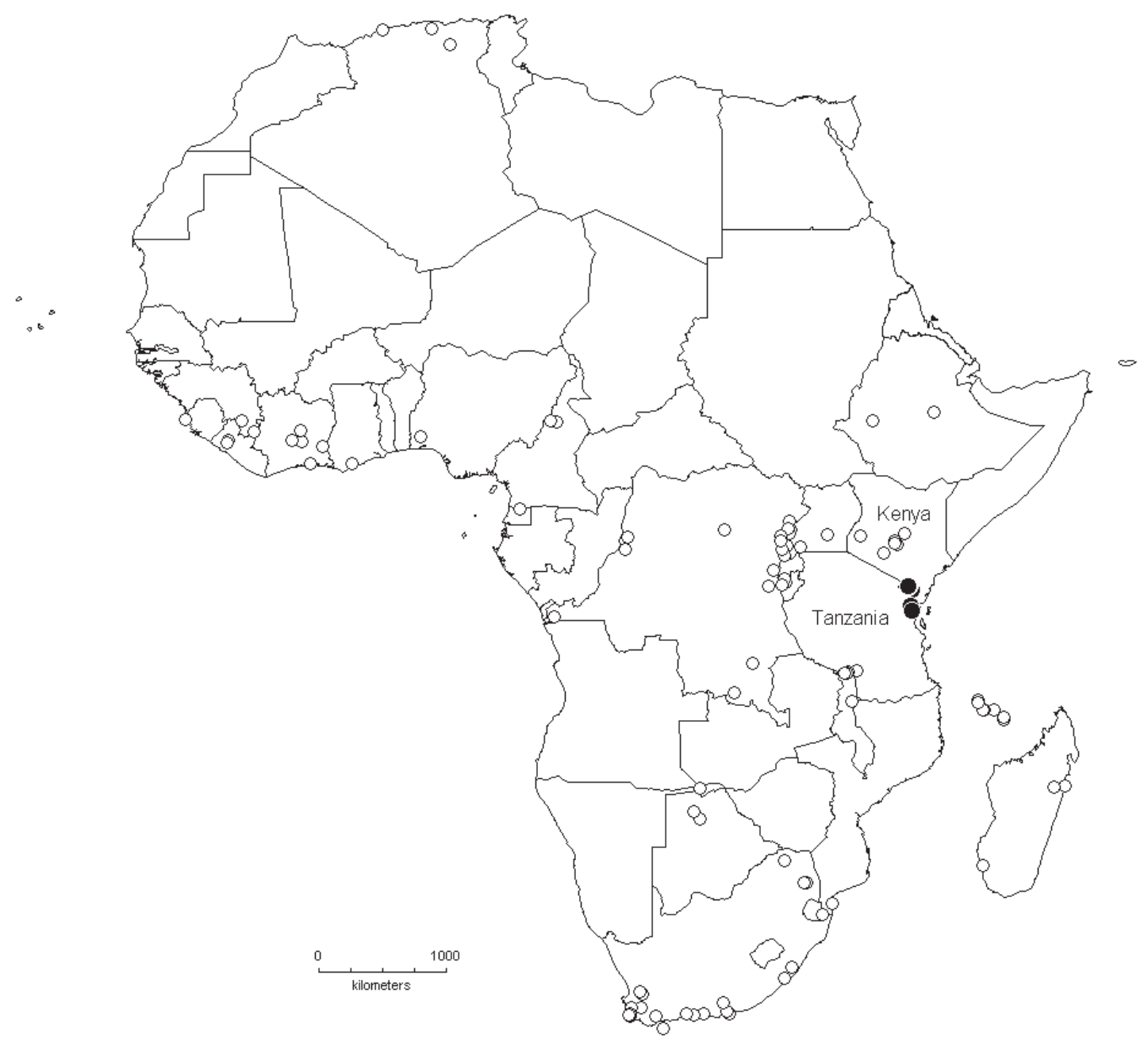

Fig. 13. Map showing all localities of georeferenced Liocranidae in the collections of MRAC: Cteniogaster gen. nov. $(\bullet)$, all other Liocranidae $(\circ)(\mathrm{n}=315)$. 

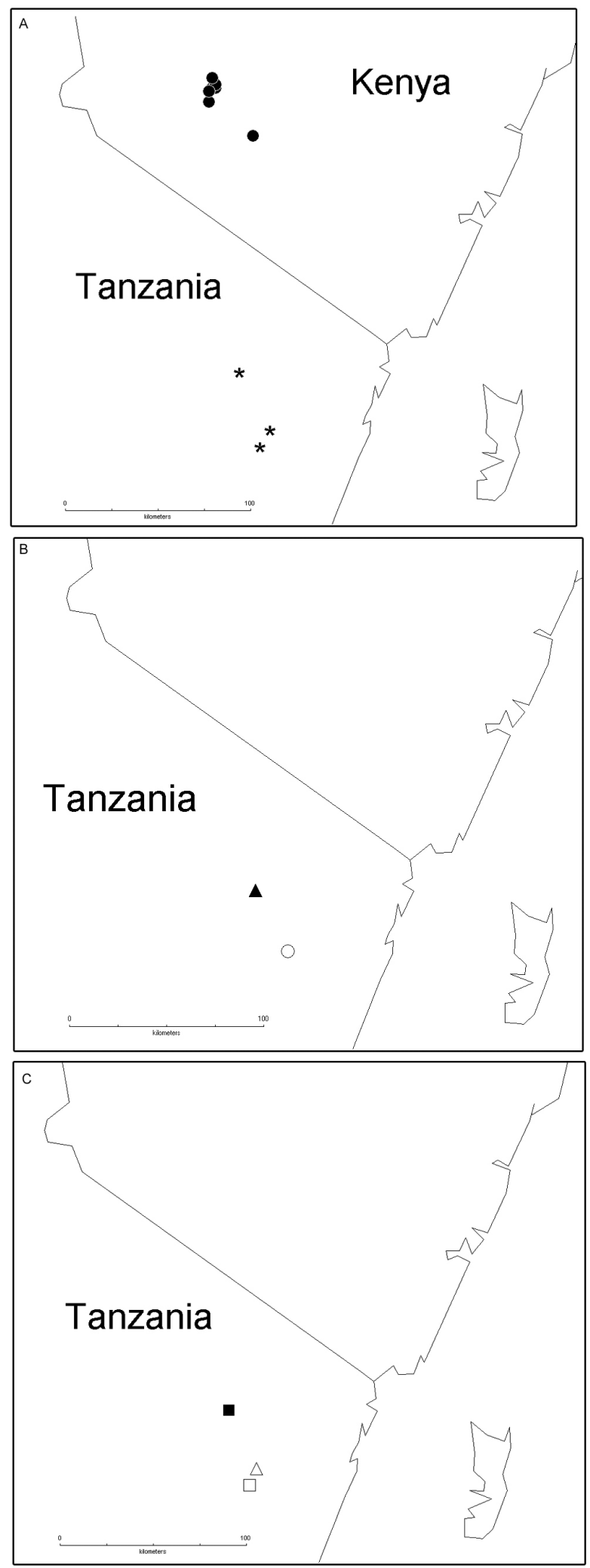

Fig. 14. Distribution maps. A. Cteniogaster hexomma sp. nov. (•), C. toxarchus gen. et sp. nov. (*).

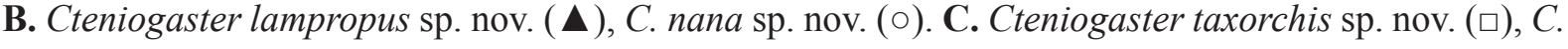
conviva sp. nov. (匹), C. sangarawe sp. nov. ( $\Delta)$. 
genera that have never been thorougly diagnosed or revised, are insufficient to judge on their affinities: these four genera are best kept in Liocranidae incertae sedis for the time being. Sesieutes Simon, 1897, will be transferred to Corinnidae, Phrurolithinae Simon, 1903 by Dankittipakul \& Deeleman-Reinhold (in press), and we propose the same transfer for the genera Jacaena Thorell, 1897, Plynnon DeelemanReinhold, 2001 and Teutamus Thorell, 1890, based on their inflated tegulum, absence of MA, modified male palpal fe, simple epigyne without anterior hood and abdominal sclerotisation (Deeleman-Reinhold 2001). A comparison of Simon's specimens of Prochora lycosiformis (O.P.-Cambridge, 1872) with the published illustrations of the male and female copulatory organs of Itatsina praticola (Bösenberg \& Strand, 1906) clearly shows that the monospecific genus Itatsina Kishida, 1930 is congeneric with the equally monospecific Prochora Simon, 1886, although both species are not identical. Consequently, Prochora praticola comb. nov. is transferred to Miturgidae Simon, 1885 here, because of its bifid RTA, combined with a cymbium with a rl groove lined with a fringe of setae and a basally inserted embolus encircling the tegulum (Song et al. 1999: fig. 238J-L). Coryssiphus Simon, 1903 and Donuea Strand, 1932 will be transferred to other families in forthcoming publications. The holotype of Montebello tenuis Hogg, 1914 was studied by Ovtsharenko (personal communication) and turned out to be a juvenile, damaged specimen with eyes and spinnerets reminiscent of Gnaphosidae Pocock, 1898. The genus is transferred to Gnaphosidae incertae sedis here.

Wunderlich's (2011: 108) proposal to include Liocranidae in Zoridae O.P.-Cambridge, 1893 and transfer Cybaeodes to Gnaphosidae is rejected because it is based on insufficient data and is not supported by a cladistic analysis. Moreover, the four zorid species studied by the authors, Zora spinimana (Sundevall, 1833), Tuxoctenus gloverae Raven, 2008, Argoctenus sp. and Hestimodema sp., apart from lacking tenent hairs and male EPGS, all share a set of characters not encountered in Liocranidae: a PER with large eyes in two rows, as in Ctenidae and Lycosidae Sundevall, 1833, PME close together, thick claw tufts, an anteriorly strongly narrowed carapace, a male palpal cymbial tip with modified thick setae as described in Raven (2008: 352), a RTA with a flattened basal membranous wing, an epigyne without anterior hood and a vulva without ST2 and with posterior globular ST1 connected to tortuous insemination ducts.

The proposals formulated here limit Liocranidae to 25 genera, including Cteniogaster gen. nov., Toxoniella and the recently described genus Vankeeria Bosselaers, 2012, of which the latter can be considered incertae sedis (Bosselaers 2012). Of these genera, four belong in Liocraninae, nine can be attributed to Cybaeodinae, and twelve remain incertae sedis, stressing the need for additional revisions and a more thourough analysis of Liocranidae and related dionychan ground spiders.

\section{Acknowledgements}

The authors are grateful to the museum curators and individual collectors mentioned above for the loan of specimens. Thanks are further due to Elise-Anne Leguin (MNHN), Julia Altmann (SMF) and Timo Pajunen (MZHF) for help in locating and sending specimens from their respective collections, and to Pablo Goloboff for explaining difference in fit value between programmes and for suggesting a way to calculate implied weighting Bremer support in TNT. The authors are also grateful to Alexandre Bonaldo, Vladimir Ovtsharenko, Norman Platnick, Martín Ramírez, Charles Haddad and Jörg Wunderlich for interesting discussions on Liocranidae and allied genera, the latter two persons also having refereed the paper. Special thanks go to Darell Ubick for initially drawing the first author's attention to the existence of this new genus by sending four specimens of Cteniogaster toxarchus sp. nov. from the CAS collection in 1998, and to Robert Raven for fruitful discussions and for the generous gift of specimens of Australian Zoridae and Miturgidae. 
BOSSELAERS J. \& JOCQUÉ R., A new afrotropical genus of Cybaeodinae (Araneae, Liocranidae)

\section{References}

Agnarsson I. \& Miller J.A. 2008. Is ACCTRAN better than DELTRAN? Cladistics 24 (6): 1032-1038. http://dx.doi.org/10.1111/j.1096-0031.2008.00229.x

Bonaldo A.B. 1997. On the new neotropical spider genus Ianduba (Araneae, Corinnidae). Iheringia, Série Zoologia 83: 165-180.

Bosmans R. 2011. On some new or rare spider species from Lesbos, Greece (Araneae: Agelenidae, Amaurobiidae, Corinnidae, Gnaphosidae, Liocranidae). Arachnologische Mitteilungen 40: 15-22. http:// dx.doi.org/10.5431/aramit4003

Bosselaers J. 2002. A cladistic analysis of Zoropsidae (Araneae), with the description of a new genus. Belgian Journal of Zoology 132 (2): 111-124.

Bosselaers J. 2009. Studies in Liocranidae (Araneae): redescriptions and transfers in Apostenus Westring and Brachyanillus Simon, as well as description of a new genus. Zootaxa 2141: 37-55.

Bosselaers J. 2012. Two interesting new ground spiders (Araneae) from the Canary Islands and Greece. Serket 13 (1-2): 83-90.

Bosselaers J. \& Jocqué R. 2000. Studies in Corinnidae: transfer of four genera and description of the female of Lessertina mutica Lawrence 1942. Tropical Zoology 13 (2): 305-325. http://dx.doi.org/10.10 $\underline{80 / 03946975.2000 .10531138}$

Bosselaers J. \& Jocqué R. 2002. Studies in Corinnidae: cladistic analysis of 38 corinnid and liocranid genera, and transfer of Phrurolithinae. Zoologica Scripta 31 (3): 241-270. http://dx.doi.org/10.1046/ j.1463-6409.2002.00080.x

Bremer K. 1988. The limits of amino acid sequence data in angiosperm phylogenetic reconstruction. Evolution 42 (4): 795-803. http://dx.doi.org/10.2307/2408870

Bremer K. 1994. Branch support and tree stability. Cladistics 10 (3): 295-304. http://dx.doi. org/10.1111/j.1096-0031.1994.tb00179.x

Bugnion E., Devine S. \& Rosenblum M. 2000. Virtual machine monitors for scalable multiprocessors. United State Patent 6075938.

Coddington J.A. \& Scharff N. 1994. Problems with zero-length branches. Cladistics 10 (4):415-423. http://dx.doi.org/10.1111/j.1096-0031.1994.tb00187.x

Dankittipakul P. \& Deeleman-Reinhold C. (In press). Delimitation of the spider genus Sesieutes Simon 1897, with descriptions of five new species from Southeast Asia (Araneae, Liocranidae). Journal of Natural History.

Deeleman-Reinhold C. 2001. Forest spiders of South East Asia. Brill, Leiden.

Dürer A. 1525. Underweysung der Messung mit den Zirckel, und Richtscheyt, in linien, ebnen und gantzen Corporen. Hieronymus Andreas Formschneider, Nuremberg. Available from http://digital.slubdresden.de/fileadmin/data/27778509X/27778509X tif/jpegs/27778509X.pdf [accessed 19 May 2009].

Forster R. 1970. The spiders of New Zealand. Vol. 3: Desidae, Dictynidae, Hahniidae, Amaurobiodidae, Nicodamidae. Otago Museum Bulletin, Otago Museum, Otago.

Goloboff P.A. 1993. Estimating character weights during tree search. Cladistics 9 (1) 83-91. http:// dx.doi.org/10.1111/j.1096-0031.1993.tb00209.x

Goloboff P.A., Farris J.S. \& Nixon K.C. 2003. TNT, Tree Analysis using New Technology. Version 1.1, sponsored by the Willy Hennig Society. Available from http://www.cladistics.com [accessed 30 Dec. 2008]. 
Goloboff P.A., Farris J.S. \& Nixon K.C. 2008a. TNT, a free program for phylogenetic analysis. Cladistics 24 (5): 774-786. http://dx.doi.org/10.1111/j.1096-0031.2008.00217.x

Goloboff P.A., Carpenter J.M., Arias J.S. \& Miranda Esquivel D.R. 2008b. Weighting against homoplasy improves phylogenetic analysis of morphological data sets. Cladistics 24 (5): 758-773. http://dx.doi. org/10.1111/j.1096-0031.2008.00209.x

Griswold C. 1993. Investigations into the phylogeny of the lycosoid spiders and their kin (Arachnida: Araneae: Lycosoidea). Smithsonian Contributions to Zoology 539: 1-39. http://dx.doi.org/10.5479/ $\underline{\text { si.00810282.539 }}$

Haddad C.R., Lyle R., Bosselaers J. \& Ramirez M.J. 2009. A revision of the endemic South African spider genus Austrachelas, with its transfer to the Gallieniellidae (Arachnida: Araneae). Zootaxa 2296: $1-38$.

Hawkins J.A., Hughes C.E. \& Scotland R.W. 1997. Primary homology assessment, characters and character states. Cladistics 13 (3): 275-283. http://dx.doi.org/10.1111/j.1096-0031.1997.tb00320.x

Jocqué R. 1991. A generic revision of the spider family Zodariidae (Araneae). Bulletin of the American Museum of Natural History 201, American Museum of Natural History, New-York.

Jocqué R. \& Dippenaar-Schoeman A.S. 2006. Spider families of the world. Sciences Zoologiques, Royal Museum for Central Africa, Tervuren.

Ledoux J.-C. \& Canard A. 1991. Initiation à l'étude systématique des araignées. J.-C. Ledoux, Aramon.

Levy G. 1990. On the cribellate spider Zoropsis lutea in Israel (Araneae, Zoropsidae). Bulletin of the British arachnological Society 8: 139-143.

Maddison W.P. 1993. Missing data versus missing characters in phylogenetic analysis. Systematic Biology 42 (4): 576-581. http://dx.doi.org/10.1093/sysbio/42.4.576

Maddison W.P., Donoghue M.J. \& Maddison D.R. 1984. Outgroup analysis and parsimony. Systematic Zoology 33: 83-103. http://dx.doi.org/10.2307/2413134

Marusik Y.M., Zheng G. \& Li S.Q. 2008. A review of the genus Paratus Simon (Araneae, Dionycha). Zootaxa 1965: 50-60.

Nixon K.C. 2002. Winclada version 1.00.08. Computer programme and documentation. Available from http://www.cladistics.com [accessed 30 Dec. 2008].

Nixon K.C. \& Carpenter J.M. 1996. On consensus, collapsibility, and clade concordance. Cladistics 12 (4): 305-321. http://dx.doi.org/10.1111/j.1096-0031.1996.tb00017.x

Penniman A. 1985. Revision of the britcheri and pugnata groups of Scotinella (Araneae, Corinnidae, Phrurolithinae) with a reclassification of phrurolithine spiders. $\mathrm{PhD}$ dissertation, The Ohio State University, Columbus. Available through University Microfilms International ( $n^{\circ} 8510623$ ).

Platnick N.I. 1990. Spinneret morphology and the phylogeny of ground spiders (Araneae, Gnaphosoidea). American Museum Novitates 2978: 1-42.

Platnick N.I. 2000 A relimitation and revision of the Australasian ground spider family Lamponidae (Araneae: Gnaphosoidea). Bulletin of the American Museum of Natural History 245, American Museum of Natural History, New-York.

Platnick N.I. 2002. A revision of the Australasian ground spiders of the families Ammoxenidae, Cithaeronidae, Gallieniellidae, and Trochanteriidae (Araneae: Gnaphosoidea). Bulletin of the American Museum of Natural History 271, American Museum of Natural History, New-York. 
BOSSELAERS J. \& JOCQUÉ R., A new afrotropical genus of Cybaeodinae (Araneae, Liocranidae)

Platnick N.I. 2012. The World Spider Catalog, version 13.0. American Museum of Natural History, New-York. Available from http://research.amnh.org/iz/spiders/catalog/ [accessed 17 September 2012].

Platnick N.I. \& Baptista R.L.C. 1995. On the spider genus Attacobius (Araneae, Dionycha). American Museum Novitates 3120: 1-9.

Platnick N.I. \& Di Franco F. 1992. On the relationships of the spider genus Cybaeodes (Araneae, Dionycha). American Museum Novitates 3053: 1-9.

Platnick N.I. \& Shadab M.U. 1975. A revision of the spider genus Gnaphosa (Araneae, Gnaphosidae) in America. Bulletin of the American Museum of Natural History 155, American Museum of Natural History, New-York.

Prentice T.R., Burger J.C., Icenogle W.R., \& Redak R.A. 1998. Spiders from Diegan coastal sage scrub (Arachnida: Araneae). Pan-Pacific Entomologist 74 (4): 181-202.

Raven R. 1998. Revision of the Australian genera of the Miturgidae with a preview of their relationships. In: XIVth International Congress of Arachnology, Abstracts 31. International Society of Arachnology.

Raven R. 2008. Revisions of Australian ground-hunting spiders: III. Tuxoctenus gen. nov. (Araneomorphae: Zoridae). Records of the Western Australian Museum 24 (4): 351-361.

Raven R.J. \& Stumkat K.S. 2002. Pteroneta Deeleman-Reinhold and a remarkable sympatric Clubiona (Clubionidae: Araneomorphae: Arachnida) in northern Australia. Memoirs of the Queensland Museum 48: 199-206.

Sanderson M.J. \& Donoghue M.J. 1989. Patterns of variation in levels of homoplasy. Evolution 43 (8): 1781-1795. http://dx.doi.org/10.2307/2409392

Schröder T., Baumscheiper M. \& Poppe E. 2000. Zubereitungen zur Behändlung keratinischer Fasern. German patent DE19907715A1.

Schulze zur Wiesche E. 2006. Moderne Haarpflegemittel. Praxis der Naturwissenschaften-Chemie in der Schule 6 (55): 14-17.

Sierwald P. 1990. Morphology and homologous features in the male palpal organ in Pisauridae and other spider families, with notes on the taxonomy of Pisauridae (Arachnida: Araneae). Nemouria 35: 1-59.

Simon E. 1892. Histoire naturelle des araignées. Tome 1, Premier fascicule. Roret, Paris. http://dx.doi. org/10.5962/bhl.title.51973

Song D.X., Zhu M.S. \& Chen J. 1999. The Spiders of China. Hebei Science and Technology Publishing House, Shijiazhuang.

Swofford D.L. 2002. PAUP* Phylogenetic Analysis under Parsimony (*and other methods) version 4.0 beta 10. Sinauer Associates, Sunderland.

Townsend V.R. Jr. \& Felgenhauer B.E. 1998. Cuticular scales of spiders. Invertebrate Biology 117 (4): 318-330. http://dx.doi.org/10.2307/3227034

Ubick D. \& Platnick N.I. 1991. On Hesperocranum, a new spider genus from Western North America (Araneae, Liocranidae). American Museum Novitates 3019: 1-12.

Ubick D. \& Vetter R.S. 2005. A new species of Apostenus from California, with notes on the genus (Araneae, Liocranidae). Journal of Arachnology 33 (1): 63-75. http://dx.doi.org/10.1636/H03-24

Warui C. \& Jocqué R. 2002. The first Gallieniellidae (Araneae) from eastern Africa. Journal of Arachnology 30 (2): 307-315. http://dx.doi.org/10.1636/0161-8202(2002)030[0307:TFGAFE]2.0.CO;2

Watrous L.E. \& Wheeler Q.D. 1981. The out-group comparison method of character analysis. Systematic Zoology 30: 1-11. http://dx.doi.org/10.1093/sysbio/30.1.1 
Wilkinson M. 1995. Arbitrary resolutions, missing entries, and the problem of zero-length branches in parsimony analysis. Systematic Biology 44 (1): 108-111. http://dx.doi.org/10.2307/2413488

Wunderlich J. 1999. Liocranoeca - eine bisher unbekannte Gattung der Feldspinnen aus Europa und Nordamerika (Arachnida: Araneae: Liocranidae). Entomologische Zeitschrift 109: 67-70.

Wunderlich J. 2004. Fossil spiders in amber and copal. Beiträge zur Araneologie 3A, B, Hirschberg.

Wunderlich J. 2008. Fossil and extant spiders. Beiträge Zur Araneologie 5, Hirschberg.

Wunderlich J. 2011. Extant and fossil spiders (Araneae). Beiträge zur Araneologie 6, Hirschberg.

Wunderlich J. 2012. The spider families of Europe: keys, diagnoses and diversity. Beiträge zur Araneologie 8, Hirschberg.

Zapata L.V. \& Ramírez M.J. 2010. A new species of the genus Paratus Simon (Araneae: Liocranidae) from Thailand. Zootaxa 2418: 65-68.

Manuscript received: 25 October 2012

Manuscript accepted: 31 January 2013

Published on: 11 March 2013

Topic editor: Koen Martens

Desk editor: Charlotte Thionois

Printed versions of all papers are also deposited in the libraries of the institutes that are members of the EJT consortium: Muséum National d'Histoire Naturelle, Paris, France; National Botanic Garden of Belgium, Meise, Belgium; Royal Museum for Central Africa, Tervuren, Belgium; Natural History Museum, London, United Kingdom; Royal Belgian Institute of Natural Sciences, Brussels, Belgium; Natural History Museum of Denmark, Copenhagen, Denmark. 
BOSSELAERS J. \& JOCQUÉ R., A new afrotropical genus of Cybaeodinae (Araneae, Liocranidae)

\section{Appendices}

Appendix 1. Leg spination schematics. A. Legend. B. Cteniogaster nana sp. nov., ô. C, F. Cteniogaster toxarchus gen. et sp. nov. D, G. Cteniogaster hexomma sp. nov. E, H. Cteniogaster conviva sp. nov.

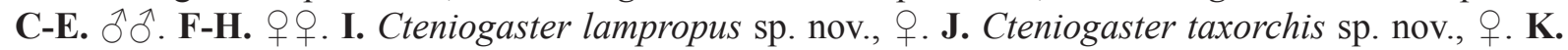
Cteniogaster sangarawe sp. nov., + . White dots are weak spines or spines that may be present or absent.

A

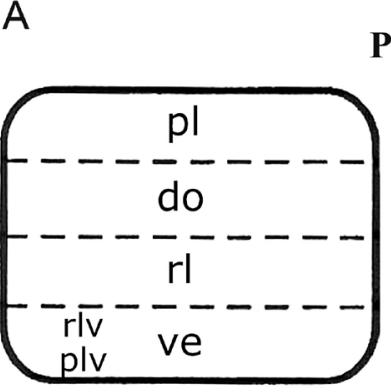

C

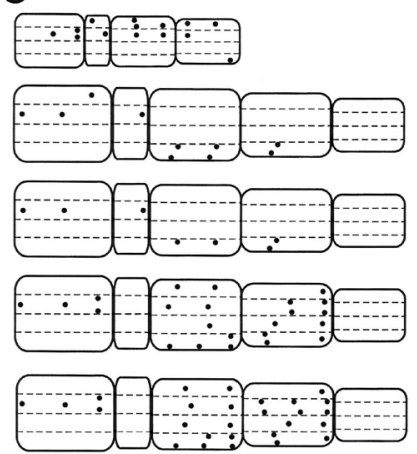

$\mathrm{F}$

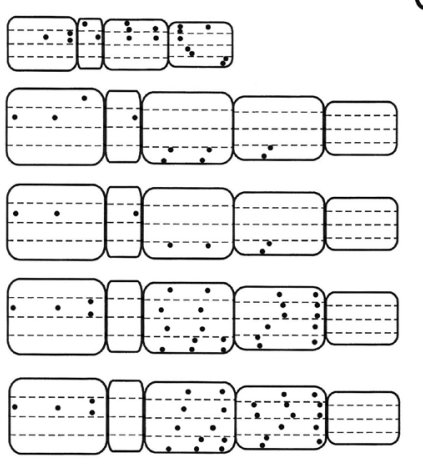

I

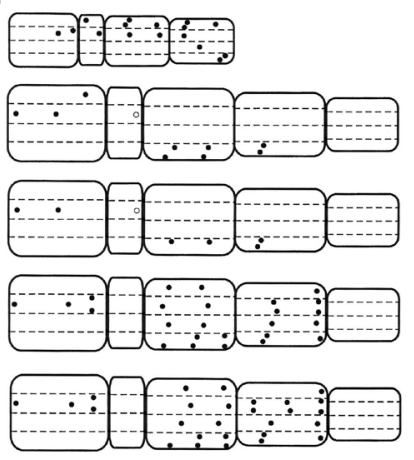

IV
Palp
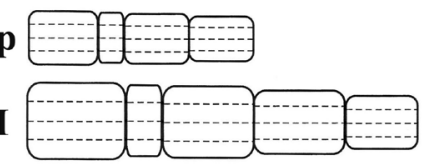

II

(1)

III

(1)

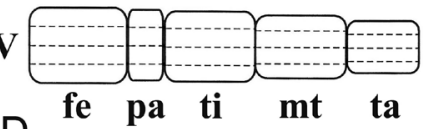

D
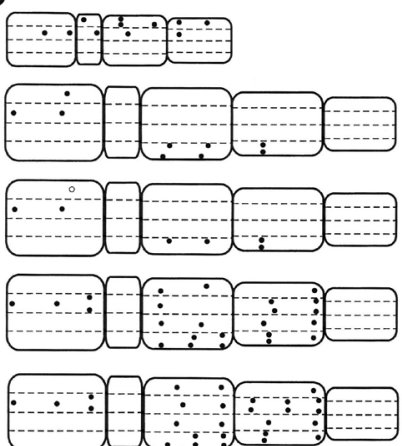

G
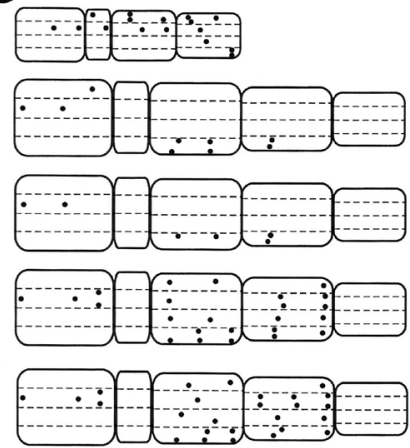

J

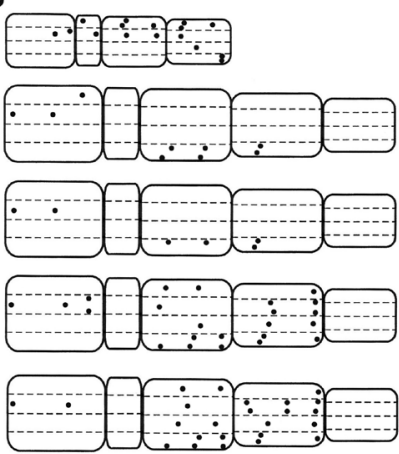

B

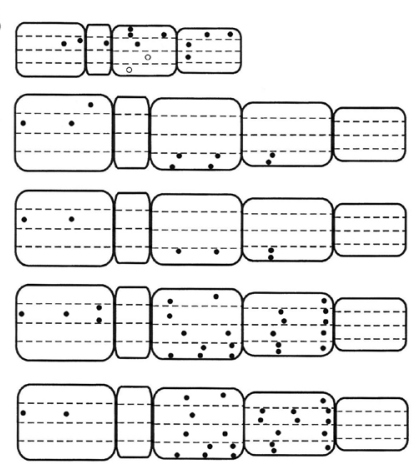

E
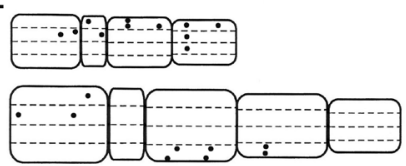

-
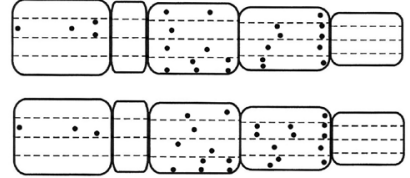

$\mathrm{H}$

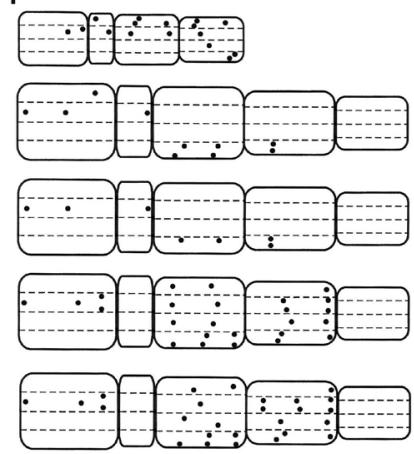

$\mathrm{K}$
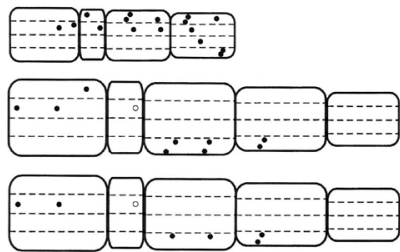

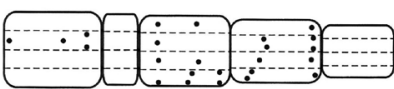

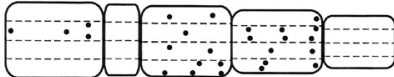


Appendix 2. Taxa examined to provide exemplar data and to allow comparison with related families, with collection data and deposition. Asterisk indicates type species of genus. Families in which taxa are placed according to prevailing views are added between square brackets $(\mathrm{Ga}=$ Gallieniellidae, $\mathrm{Li}=$ Liocranidae, $\mathrm{Co}=$ Corinnidae, $\mathrm{Zo}=$ Zoridae $)$.

\section{Outgroup}

Corinna nitens (Keyserling, 1891) [Co]; 2 ồ 1 q : Brazil, Rio Grande do Sul, Novo Hamburgo, 27 Feb. 1979, T. de Lema leg. (MCN 8558); 1 đ: Rio Grande do Sul, São Leopoldo, 27 Jun. 1987, L. de A. Moura leg. (MCN 16884); 1 q: Rio Grande do Sul, São Francisco de Paula, Barragem dos Bugres, 25 Nov. 1998, L. de A. Moura leg. (MCN 30653).

Creugas bajulus (Gertsch, 1942) [Co]; 1 ठ̊: USA, California, San Diego County, Miramar Naval Air Station, coastal sage scrub, pitfall trap, May-Jun. 1996, T. Prentice leg. (UCR); 1 q: same data, Aug. 1996 (UCR) (Prentice et al. 1998).

\section{Ingroup}

Agraecina lineata* (Simon, 1878) [Li] ; 5 ô ổ: France, Corsica and Menton (MNHN 2095); 1 q: France, Les Saintes Maries, "Collection Berland, entrée n 4.25.9.62" (MNHN).

Agroeca brunnea* (Blackwall, 1833) [Li]; 1 §̊: Belgium, Beerse, "De Schrieken", pitfall trap in pine wood with bracken, 20 Feb. 1995, J. Bosselaers leg. (CJB 1003); 1 ㅇ: same locality, pitfall trap in alder marsh, 2 Dec. 1994, J. Bosselaers leg. (CJB 882).

Agroeca cuprea [Li]; 1 ${ }^{\lambda}$ : Belgium, Hautes Fagnes, no further data (CHK); 1 : France, Département Var, between Pierrefeu and Collobrières, under wood and stones in open Quercus suber and Pinus forest, 4313'53.4”'N 6¹4'1.6”'E, alt. 100 m. 17 Mar. 2006, A Schönhofer leg. (SMF).

Agroeca parva Bosmans, 2011 [Li]; 1 ठో: Greece, Chios, Mount Pelinion, pitfall trap, 26 Sep.-10 Oct. 2009 (CARS); 1 : Greece, Chios, Padoukios, pitfall trap, 6-22 May 2009 (CARS).

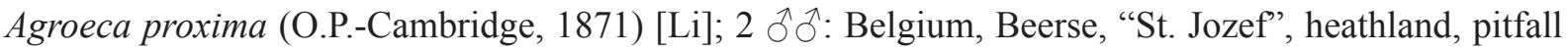
trap, 4 Oct. 1985 (CJB 472a); 1 q: Belgium, Beerse, "De Schrieken”, Calluna heathland, pitfall trap, 20 Nov. 1994, J. Bosselaers leg., (CJB 837b)

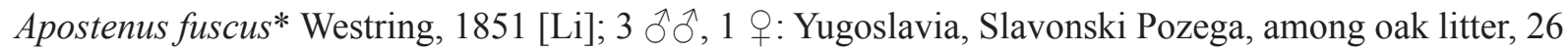
Mar. 1972, C. Deeleman-Reinhold leg. (RMNH).

Apostenus humilis Simon, 1932 [Li]; 1 §ึ: no further data (MNHN25305); 21 우: “Apostenus humilis E.S. in litteris et det. Banyuls 09” (MNHN25).

Cteniogaster conviva sp. nov.; 1 o, 1 (holotypus and paratypus): Tanzania, Tanga, West Usambara Mountains, Mazumbai Forest, sifting litter, 449’S 38³0'E, alt. 1400-1800 m. 11-20 Nov. 1998, Charles Griswold, Nikolaj Scharff and Darrell Ubick leg. (ZMUC).

Cteniogaster hexomma sp. nov.; $1 \hat{\jmath}, 1$ (holotypus and paratypus): Kenya, Taita Hills, Ngangao forest, mixed forest, pitfall trap, $03^{\circ} 22^{\prime} \mathrm{S} 038^{\circ} 21^{\prime} \mathrm{E}$, alt. $1820 \mathrm{~m}$. 15 Jul. 1998, Lucy Rogo leg. (MRAC 208888).

Cteniogaster toxarchus* sp. nov.; 17 ふ઼่, 25 우 (paratypi): Tanzania, East Usambara Mountains, Amani, sifting litter, 55.7’S 38³8’E, alt. 950 m. 28 Oct.- 9 Nov. 1995, Charles Griswold, Nikolaj Scharff and Darrell Ubick leg. (ZMUC). 
BOSSELAERS J. \& JOCQUÉ R., A new afrotropical genus of Cybaeodinae (Araneae, Liocranidae)

Cybaeodes mallorcensis Wunderlich, 2008 [Li]; 1 ठ (holotypus): Spain, Mallorca, South of Embalse de Cuber, under stone, Aug., J. Wunderlich leg. (SMF 60217); 1 q (paratypus): Spain, Mallorca, Valldemossa, Jul., J. Wunderlich leg. (SMF 60218).

Cybaeodes marinae Di Franco, 1989 [Li]; 1 ภ: Italy, Sicily, Nebrodi, E. slope of Monte Pagano, $550 \mathrm{~m}$, mixed shrub with Quercus suber and Q. gussonei, Sep. 1988 (AMNH); 1 ภ , 1 우: Italy, Lazio, Rome, Castelporziano, oakwood, 13 Oct. 1986, Bonavita leg. (AMNH).

Hesperocranum rothi* Ubick \& Platnick, 1991 [Li]; 1 O : USA, California, Tulare County, 2.5 mi E of Cal. Hot Springs, 15 Sep. 1959, V. Roth \& W. Gertsch leg. (AMNH); 1 \%: California, Fresno County, Kings Cyn. Nat. Park, Cedar Grove, 13 Sep. 1959, V. Roth \& W. Gertsch leg. (AMNH).

Liocranoeca striata* (Kulczynski, 1882) [Li]; 1 đో: France, Southern France, coastal area (MNHN 6147); 1 q: France, Corsica and Menton (MNHN 2095).

Liocranum giersbergi Kraus, 1955 [Li]; 1 ภ, 2 우옹 Italy, Sardinia, Villanova Strisaili, pitfall trap at lakeside in wood, 23 May 1997, J. van Keer leg. (CJK 1757); 1 t (holotypus): Italy, Sardinia, Bunorva, alt. 500 m. 15 Apr. 1952, K. Schüllbächer leg. (SMF 8971/1).

Liocranum rupicola* (Walckenaer, 1830) [Li]; 1 ${ }^{\lambda}, 1$ : Italy, Lazio, Latina, street to summit of the Monte Circea, oak forest, sieving litter under wood and stones. $41^{\circ} 13^{\prime} 58.3^{\prime \prime} \mathrm{N} \mathrm{13} 3^{\circ} 04^{\prime} 59.6^{\prime \prime} \mathrm{E}$, alt. $229 \mathrm{~m}$. 9 Apr. 2006, A. Schönhofer leg. (SMF “131d”).

Mesiotelus cyprius Kulczynski, 1908 [Li]; 1 ภ, 8 우우 Cyprus, Troodos Mountain, 1600 m, under stones in pine forest, 28 Oct. 1981, P.R. Deeleman leg. (RMNH); 7 우: Crete, Zoniana, outside Sventoni Cave, 700 m, under stones, 6 Apr. 1996, J. Bosselaers leg. (CJB 1047).

Mesiotelus tenuissimus* (L. Koch, 1866) [Li]; 2 đิ ô: Greece, Naxos, 16 Oct. 1984, Parashi leg. (SMF); 1 Q\#f: Syria, Homs province, Qal' at al-Husn, ruins and abandoned cemetery NW of the castle. 8 Mar. 1979, R. Kinzelbach leg. (SMF).

Neoanagraphis chamberlini* Gertsch \& Mulaik, 1936 [Li]; 2 ふぇ: USA, New Mexico, Socorro County, Sevilleta 20 mi N Socorro, 1500-2200 m, 1989-1992, S Brantley leg. (UCR, now in CJB); 1 ㅇ: same data (UCR).

Sagana rutilans* Thorell, 1875 [Li]; $1 \overbrace{}^{\lambda}$ : Bosnia and Herzegovina, Prisoje area, Livno near Tomisiovgrad, Camp Grabovica, collected at night under stones at small stream, $43.69512^{\circ} \mathrm{N} 17.09543^{\circ} \mathrm{E}$, alt. $875 \mathrm{~m}$. 3-5 Sep. 2009, A. Schönhofer leg. (SMF); 5 우: “Gallia” (MNHN 2098).

Scotina celans (Blackwall, 1841) [Li]; 1 ภ, 4 q $q$ : France, Banyuls (MNHN); 2 qo+: France, Saint Gilles (MNHN); 1 O: Yugoslavia, Island Cres, Jama, Lipici Cave, 1 Apr. 1972, C.L. \& P.R. Deeleman leg. (RMNH).

Scotina gracilipes* (Blackwall, 1859) [Li]; 1 Oో, 2 우: Denmark, Anholt, plantation, 11 Aug. 1946, Ø.

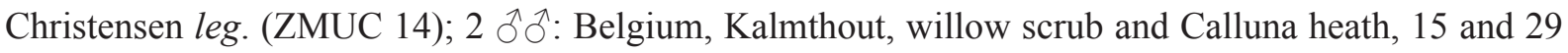
Nov. 1976, P. Baert leg. (CJK 862).

Scotina palliardii (L. Koch, 1881) [Li]; 5 $\widehat{\jmath}, 2$ + q : Finland, North Karelia, Llomantsi, Heinäsuo, 26E, 5 Jun. 2007, O. Loukola and O. Autio leg. (MZHF 98274). 
Toxoniella rogoae Warui \& Jocqué, 2002 [Ga]; 1 đ (holotypus): Kenya, Taita Hills, Ngangao forest, mixed forest, pitfall trap, 0322'S 38²1’E, alt. 1820 m. 15 Jul. 1998, Lucy Rogo leg. (MRAC 209914); 1 (paratypus): same data (MRAC 209661).

Toxoniella taitensis* Warui \& Jocqué, 2002 [Ga]; 1 ภ, 1 q (paratypi): Kenya, Taita Hills, Ngangao forest, $03^{\circ} 22^{\prime}$ S $38^{\circ} 21^{\prime} \mathrm{E}$, alt. 1820 m. 15 Jul. 1998, L. Rogo leg. (MRAC 208887).

\section{Additional specimens studied}

Argoctenus sp. [Zo]; 1 § : Australia, SE Queensland, Lake Broadwater via Dalby, pitfall traps, 24 Nov. 1985-3 Jan. 1986, M. Bennie leg. (CJB).

Argoctenus sp. [Zo]; 1 O: Australia, SE Queensland, Lake Broadwater via Dalby, pitfall traps, 19 Feb.26 Mar. 1985, M. Bennie leg. (CJB).

Austrachelas natalensis Lawrence, 1942 [Ga]; 1 đ , 2 우: South Africa, Natal, St. Lucia, Fanies Island, woodland edge with grass, pitfall traps, 21-25 Feb. 1990, M. Alderweireldt \& R. Jocqué leg. (MRAC 171752).

Austrachelas pondoensis Haddad et al., 2009 [Ga]; 1 ภ, 8 우: South Africa, Lusikisiki District, Transkei

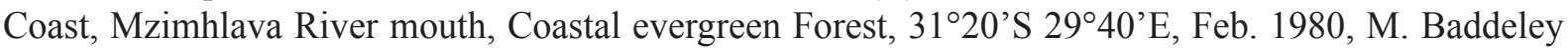
leg. (MRAC 163974).

Drassodella sp. [Ga] 1 ' : Mozambique, Inhaca, Coastal woodland, pitfalls, $26^{\circ} 01^{\prime} \mathrm{S} 32^{\circ} 54^{\prime} \mathrm{E}, 13-30$ Apr. 1994, T. Steyn leg. (MRAC 209887).

Gallieniella jocquei Platnick, 1984 [Ga]; 1 §̂, 1 ค: Comoros, Mohéli, Miringoni, plantations d'Ilang Ilang, $12^{\circ} 15^{\prime} \mathrm{S} 43^{\circ} 45^{\prime} \mathrm{E}$, Mango and other trees at adge of plantation, pitfall traps, 5-13 Nov. 1983, R. Jocqué leg. (MRAC 160925).

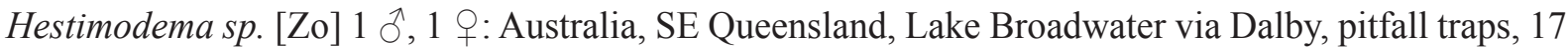
May-24 Nov. 1985, M. Bennie leg. (CJB).

Tuxoctenus gloverae Raven, 2008 [Zo] 1 ภ̊: Australia, SE Queensland, Burbank, Buhot Creek, riparian forest, $27^{\circ} 35.5^{\prime} \mathrm{S} 153^{\circ} 10.3^{\prime} \mathrm{E}, 50 \mathrm{~m}$, pitfall traps, 30 Jan.-1 Mar. 2004 (CJB).

Tuxoctenus gloverae Raven, 2008 [Zo] 1 ㅇ: Australia, SE Queensland, summit of Mount Gayndah, 2536'S 151³2'E, 340 m, pitfall traps, 27 Jan.-2 Jun. 1999, Montelth \& Thompson leg. (CJB).

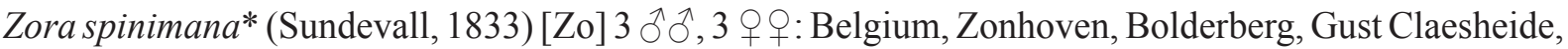
mixed forest with Calluna heath, pitfall traps, 27 Nov. 1983-21 Mar. 1984, J. Bosselaers leg. (CJB 0045). 
BOSSELAERS J. \& JOCQUÉ R., A new afrotropical genus of Cybaeodinae (Araneae, Liocranidae)

Appendix 3. Character-taxon matrix.

\begin{tabular}{|c|c|c|c|c|c|c|c|c|c|c|c|c|c|c|c|c|c|c|c|c|}
\hline Golo & ?ु? & 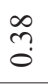 & $\stackrel{n}{\mathfrak{o}}$ & $\stackrel{n}{\stackrel{2}{0}}$ & $\stackrel{8}{8}$ & $\stackrel{n}{\mathfrak{0}}$ & $\stackrel{n}{\mathfrak{0}}$ & $\underset{-}{8}$ & $\underset{-}{8}$ & $\begin{array}{l}8 \\
0 \\
0\end{array}$ & $\begin{array}{l}\stackrel{8}{0} \\
\stackrel{0}{0}\end{array}$ & $\begin{array}{l}0 \\
0\end{array}$ & $\begin{array}{l}\infty \\
\stackrel{0}{0} \\
0\end{array}$ & $\stackrel{\text { f̊ }}{\circ}$ & $\begin{array}{l}8 \\
0 \\
0\end{array}$ & $\begin{array}{l}\infty \\
\stackrel{\infty}{0} \\
\stackrel{n}{0}\end{array}$ & $\underset{-}{8}$ & $\underset{-}{8}$ & $\begin{array}{l}8 \\
\stackrel{0}{0}\end{array}$ & $\stackrel{?}{\stackrel{?}{0}}$ \\
\hline ri & 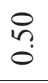 & $\frac{1}{0}$ & $\begin{array}{l}\infty \\
\stackrel{0}{0}\end{array}$ & $\stackrel{8}{\circ}$ & $\stackrel{8}{\circ}$ & $\begin{array}{l}\infty \\
\infty \\
0\end{array}$ & $\stackrel{n}{\stackrel{0}{0}}$ & $\stackrel{8}{\circ}$ & $\stackrel{8}{\circ}$ & $\begin{array}{l}\hat{\sigma} \\
0\end{array}$ & ๙̋ & $\begin{array}{l}\text { ? } \\
\text { ? }\end{array}$ & $\begin{array}{l}\text { ? } \\
0\end{array}$ & రై & $\begin{array}{l}\infty \\
\stackrel{0}{0}\end{array}$ & $\begin{array}{l}\infty \\
\text { ? } \\
?\end{array}$ & $\stackrel{8}{\circ}$ & $\stackrel{8}{\circ}$ & $\frac{\infty}{\stackrel{0}{0}}$ & $\stackrel{n}{0}$ \\
\hline ci & $\stackrel{ \pm}{\overrightarrow{0}}$ & $\stackrel{5}{\circ}$ & 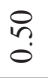 & \begin{tabular}{l}
$\stackrel{n}{n}$ \\
\hdashline
\end{tabular} & $\stackrel{8}{8}$ & $\begin{array}{l}\stackrel{n}{n} \\
\stackrel{n}{0}\end{array}$ & $\stackrel{n}{n}$ & $\stackrel{8}{8}$ & $\stackrel{8}{8}$ & $\stackrel{m}{m}$ & $\stackrel{m}{?}$ & $\stackrel{m}{0}$ & $\begin{array}{l}\infty \\
\stackrel{n}{0} \\
?\end{array}$ & $\stackrel{\mathscr{P}}{\stackrel{0}{0}}$ & 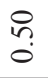 & $\stackrel{ }{\circ}$ & $\stackrel{8}{8}$ & $\stackrel{8}{8}$ & 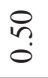 & $\stackrel{m}{?}$ \\
\hline Steps & $\underline{m}$ & $r$ & 으 & $=$ & $n$ & $\infty$ & $n$ & $\infty$ & $n$ & $r$ & $\simeq$ & $n$ & 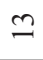 & 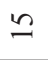 & $\simeq$ & $a$ & $\infty$ & $\infty$ & $=$ & 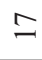 \\
\hline Toxoniella taitensis & 0 & - & - & 0 & a. & 0 & 0 & - & 0 & 0 & 0 & 0 & - & 0 & $\sigma$. & $\sigma$. & - & - & $N$ & 一 \\
\hline Toxoniella rogoae & 0 & - & - & 0 & a. & 0 & 0 & - & 0 & 0 & o & ○ & ○ & 0 & a. & 0 & - & - & a. & 一 \\
\hline Scotina palliardii & - & - & - & 0 & a. & - & 0 & - & - & a. & 一 & 0 & $m$ & $m$ & $\sim$ & 0 & - & - & $\sim$ & 0 \\
\hline Scotina gracilipes & - & - & - & 0 & a. & - & 0 & - & - & - & - & o & m & n & v & 0 & - & - & v & 0 \\
\hline Scotina celans & - & - & - & $\circ$ & a. & - & 0 & - & - & - & - & ○ & m & n & N & a. & - & - & N & 0 \\
\hline Sagana rutilans & 0 & 0 & 0 & - & - & 0 & 0 & 0 & ○ & 0 & ○ & ○ & $m$ & $m$ & $\sim$ & 0 & 0 & 0 & - & $N$ \\
\hline Neoanagraphis chamberlini & 0 & 0 & - & - & 0 & 0 & 0 & - & 0 & - & 一 & 一 & 一 & - & - & 0 & - & - & N & $N$ \\
\hline Mesiotelus tenuissimus & - & - & 0 & - & - & 0 & 0 & 0 & 0 & 0 & ○ & ○ & - & - & - & 0 & 0 & 0 & - & - \\
\hline Mesiotelus cyprius & - & - & 0 & - & - & 0 & 0 & 0 & 0 & 0 & 0 & 0 & - & - & - & 0 & 0 & 0 & - & - \\
\hline Liocranum rupicola & 0 & - & 0 & - & - & 0 & 0 & 0 & 0 & 0 & o & o & v & $\sim$ & $\sim$ & 0 & 0 & 0 & - & $\sigma$ \\
\hline Liocranum giersbergi & 0 & - & 0 & - & - & 0 & 0 & 0 & 0 & $\sigma$. & 0 & 0 & N & N & N & 0 & 0 & 0 & - & $\mathrm{N}$ \\
\hline Liocranoeca striata & 0 & 0 & - & 0 & a. & 0 & 0 & - & 0 & 0 & 一 & 一 & 一 & - & - & - & - & - & N & $\sim$ \\
\hline Hesperocranum rothi & - & - & 0 & - & - & 0 & 0 & 0 & 0 & 0 & o & o & o & 0 & 0 & 0 & 0 & 0 & 0 & 0 \\
\hline Cybaeodes marinae & 0 & 0 & - & - & 0 & 0 & 0 & - & 0 & $\circ$ & - & ○ & $\sim$ & $\sim$ & $\sim$ & - & - & - & - & - \\
\hline Cybaeodes mallorcensis & 0 & 0 & - & - & 0 & 0 & 0 & - & 0 & 0 & 0 & 0 & $\sim$ & $N$ & N & - & - & - & - & - \\
\hline Cteniogaster toxarchus & - & - & - & 0 & a. & - & - & - & 0 & 0 & o & o & - & - & - & - & - & - & - & o \\
\hline Cteniogaster hexomma & - & - & - & 0 & a. & - & - & - & 0 & 0 & ○ & ○ & - & - & - & - & - & - & - & 0 \\
\hline Cteniogaster conviva & - & - & - & 0 & ๙. & - & - & - & 0 & 0 & 0 & 0 & 一 & - & - & - & - & - & - & 0 \\
\hline Apostenus humilis & 0 & 0 & 0 & 0 & a. & - & - & - & - & 0 & - & 0 & $\sim$ & N & $N$ & 0 & - & - & $N$ & 0 \\
\hline Apostenus fuscus & - & - & 0 & 0 & a. & - & - & - & - & 0 & - & 0 & N & N & $\sim$ & 0 & - & - & $\sim$ & 0 \\
\hline Agroeca proxima & - & - & - & 0 & a. & 0 & 0 & - & 0 & - & 一 & 0 & 一 & - & - & 0 & - & - & $\sim$ & $N$ \\
\hline Agroeca parva & 0 & - & - & 0 & a. & 0 & 0 & - & 0 & - & - & o & - & - & - & - & - & - & $\sim$ & N \\
\hline Agroeca cuprea & - & - & - & 0 & a. & 0 & 0 & - & 0 & 0 & - & - & - & - & - & - & - & - & $\sim$ & N \\
\hline Agroeca brunnea & - & - & - & 0 & a. & 0 & 0 & - & ○ & - & 一 & 一 & 一 & - & - & 0 & - & - & $\sim$ & $N$ \\
\hline Agraecina lineata & 0 & - & - & 0 & ๙. & 0 & 0 & - & 0 & - & - & - & - & - & - & 0 & - & - & $\sim$ & N \\
\hline Creugas bajulus & 0 & 0 & 0 & - & 0 & 0 & 0 & 0 & 0 & 0 & - & 0 & $N$ & $N$ & $N$ & 0 & 0 & 0 & $N$ & - \\
\hline Corinna nitens & - & - & 0 & - & 0 & 0 & 0 & 0 & 0 & 0 & 一 & 0 & - & $N$ & a. & - & 0 & 0 & $N$ & - \\
\hline Characters & - & $N$ & $m$ & $\nabla$ & $n$ & 0 & $r$ & $\infty$ & $a$ & 웅 & $=$ & $\simeq$ & 9 & \pm & 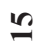 & $\stackrel{0}{ }$ & $\Xi$ & $\infty$ & 2 & శ్ \\
\hline
\end{tabular}




\begin{tabular}{|c|c|c|c|c|c|c|c|c|c|c|c|c|c|c|c|c|c|c|c|c|}
\hline Golo & $\begin{array}{l}\stackrel{n}{0} \\
0\end{array}$ & $\begin{array}{l}0 \\
0 \\
0\end{array}$ & $\stackrel{n}{\circ}$ & $\stackrel{n}{\stackrel{n}{0}}$ & $\stackrel{?}{\circ}$ & లి & $\stackrel{n}{\circ}$ & $\stackrel{8}{\circ}$ & $\begin{array}{l}\text { ?ִ } \\
0\end{array}$ & $\stackrel{?}{\leftrightarrow}$ & $\stackrel{n}{\circ}$ & $\stackrel{8}{\circ}$ & $\stackrel{8}{8}$ & $\stackrel{n}{\circ}$ & $\stackrel{8}{\circ}$ & $\stackrel{8}{8}$ & $\begin{array}{l}\text { ?ִ } \\
0\end{array}$ & $\stackrel{8}{8}$ & $\stackrel{8}{\circ}$ & $\stackrel{8}{\circ}$ \\
\hline ri & $\hat{\sigma}$ & $\stackrel{?}{\circ}$ & $\begin{array}{l}\infty \\
\infty \\
0\end{array}$ & $\begin{array}{l}\infty \\
\infty \\
0\end{array}$ & $\stackrel{t}{0}$ & @̊ & $\begin{array}{l}\text { ㅇ } \\
\text { n̊ }\end{array}$ & $\stackrel{n}{\stackrel{0}{0}}$ & $\stackrel{m}{0}$ & $\begin{array}{l}\text { Pn } \\
\text { no }\end{array}$ & ŏ & $\begin{array}{l}\text { ㅇ. } \\
\text { ? }\end{array}$ & $\stackrel{8}{8}$ & ふু & $\frac{\infty}{\stackrel{0}{0}}$ & $\underset{-}{\stackrel{8}{0}}$ & $\hat{\sigma}$ & 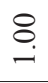 & $\begin{array}{l}\text { ô } \\
\stackrel{0}{0}\end{array}$ & 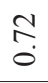 \\
\hline ci & $\begin{array}{l}\text { in } \\
\text { ñ }\end{array}$ & $\stackrel{+}{+}$ & ?̊? & ?̊? & $\stackrel{\leftrightarrow}{\leftrightarrow}$ & $\stackrel{\text { ָ̦ }}{0}$ & ?̊ & $\stackrel{m}{0}$ & $\stackrel{+}{+}$ & ָ̊ & ?̊? & $\stackrel{m}{?}$ & $\stackrel{8}{\circ}$ & ?̊? & $\stackrel{m}{?}$ & $\stackrel{8}{8}$ & $\stackrel{\sim}{a}$ & $\stackrel{8}{8}$ & $\stackrel{m}{?}$ & $\begin{array}{l}\text { n? } \\
\text { ñ }\end{array}$ \\
\hline Steps & $\simeq$ & $\simeq$ & $r$ & 6 & $\Xi$ & $\simeq$ & $m$ & $a$ & $\stackrel{n}{=}$ & $a$ & $\stackrel{n}{=}$ & in & 6 & $\stackrel{n}{=}$ & $\stackrel{0}{=}$ & $\sim$ & $\stackrel{0}{=}$ & $n$ & $\simeq$ & $a$ \\
\hline Toxoniella taitensis & $N$ & $N$ & - & - & N & - & - & - & - & - & - & - & 0 & - & - & 0 & - & $N$ & - & - \\
\hline Toxoniella rogoae & N & $N$ & - & 0 & $\sim$ & - & - & - & - & - & - & - & 0 & - & - & 0 & - & $N$ & - & - \\
\hline Scotina palliardii & $N$ & $\sim$ & - & - & $\sim$ & $\sim$ & 0 & - & - & 0 & 0 & $\curvearrowright$ & 0 & 0 & 0 & 0 & 0 & $\curvearrowright$ & 0 & 0 \\
\hline Scotina gracilipes & $\mathrm{N}$ & N & - & - & v & - & - & - & - & 0 & 0 & a. & 0 & 0 & 0 & 0 & 0 & a. & 0 & 0 \\
\hline Scotina celans & $\sigma$ & $N$ & 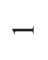 & - & $\sim$ & $\neg$ & - & $\neg$ & $\neg$ & 0 & 0 & 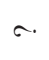 & 0 & 0 & 0 & 0 & 0 & $\sigma$ & 0 & 0 \\
\hline Sagana rutilans & $m$ & - & - & - & $m$ & 一 & - & - & $\sim$ & - & - & - & - & - & - & 0 & - & $\sim$ & - & - \\
\hline Neoanagraphis chamb & $m$ & 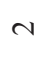 & 0 & - & $m$ & - & - & - & $\sim$ & - & 0 & ๙. & 0 & - & - & 0 & 0 & r. & 0 & 0 \\
\hline Mesiotelus tenuissimus & $N$ & $\neg$ & - & 0 & $m$ & - & - & 0 & $\sim$ & - & - & 0 & - & - & $\neg$ & 0 & 0 & $\sigma$. & $\neg$ & 0 \\
\hline Mesiotelus cyprius & v & - & - & 0 & n & - & - & 0 & - & - & - & 0 & - & - & - & 0 & ○ & a. & - & 0 \\
\hline Liocranum rupicola & a. & - & - & 0 & $m$ & $\sim$ & - & 0 & $\sim$ & - & - & 0 & - & - & - & 0 & 0 & a. & - & 0 \\
\hline Liocranum giersbergi & $\sigma$ & - & - & 0 & $m$ & $\sim$ & - & 0 & $\sim$ & - & - & 0 & - & - & - & 0 & 0 & $\sigma$. & $\neg$ & 0 \\
\hline Liocranoeca striata & $m$ & $\sim$ & 0 & - & n & - & - & - & $\sim$ & 0 & ○ & 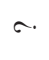 & ○ & 0 & ○ & 0 & 0 & $\curvearrowright$ & 0 & 0 \\
\hline Hesperocranum rothi & 0 & 0 & - & 0 & 0 & 0 & 一 & 0 & $\sim$ & 0 & 0 & a. & - & - & - & 0 & 0 & a. & - & - \\
\hline Cybaeodes marinae & $c$ & - & - & - & - & - & - & - & - & - & - & 0 & 0 & - & - & 0 & - & N & - & - \\
\hline Cybaeodes mallorcensis & $\sigma$ & - & - & - & - & - & - & - & - & - & - & 0 & 0 & - & - & 0 & - & $\sim$ & - & - \\
\hline Cteniogaster toxarchus & - & $\sim$ & - & - & $\sim$ & $\sim$ & - & - & - & - & - & 0 & 0 & 0 & 0 & 0 & - & - & - & 0 \\
\hline Cteniogaster hexomma & - & $\sim$ & - & - & $\sim$ & - & - & 0 & - & - & - & 0 & 0 & 0 & 0 & 0 & - & - & - & 0 \\
\hline Cteniogaster conviva & - & $\sim$ & - & - & $\sim$ & 0 & - & 0 & - & - & - & $\circ$ & 0 & 0 & 0 & 0 & - & - & - & 0 \\
\hline Apostenus humilis & a. & 0 & - & - & $\sim$ & 0 & - & 0 & $\sim$ & 0 & 0 & ๙. & 0 & 0 & 0 & 0 & - & 0 & 0 & 0 \\
\hline Apostenus fuscus & $\sigma$ & - & - & - & $m$ & $N$ & - & 0 & v & 0 & 0 & a. & 0 & 0 & 0 & 0 & - & 0 & 0 & 0 \\
\hline Agroeca proxima & $c$ & $\sim$ & 0 & - & $m$ & $\curvearrowright$. & 一 & - & $\sim$ & - & 0 & a. & 0 & 0 & - & 0 & 0 & $\curvearrowright$ & - & - \\
\hline Agroeca & $m$ & $\sim$ & 0 & - & $m$ & $\sim$ & - & - & $\sim$ & - & 0 & a. & 0 & 0 & - & 0 & 0 & a. & 0 & 0 \\
\hline Agroeca cuprea & $m$ & $N$ & 0 & - & $m$ & 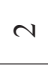 & - & - & N & - & 0 & r. & 0 & 0 & - & 0 & 0 & $\curvearrowright$ & 0 & 0 \\
\hline Agroeca brunnea & 7 & $\sim$ & 0 & 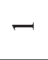 & $m$ & 一 & 一 & - & $\sim$ & - & 0 & $\curvearrowright$ & 0 & 0 & - & 0 & 0 & $\curvearrowright$ & 0 & 0 \\
\hline Agraecina lineata & $m$ & $\sim$ & 0 & - & $m$ & - & - & - & $\sim$ & 0 & 0 & a. & 0 & 0 & 0 & 0 & 0 & $\curvearrowright$ & 0 & 0 \\
\hline Creugas bajulus & $N$ & - & - & - & - & 0 & 0 & - & 0 & - & - & - & 0 & - & - & - & 0 & a. & - & $N$ \\
\hline Corinna nitens & $N$ & 0 & - & - & $\sim$ & 0 & 0 & 一 & 一 & 0 & - & - & 0 & - & - & - & 0 & $\curvearrowright$ & 0 & $\sim$ \\
\hline Characters & $\bar{\sim}$ & $N$ & $N$ & $\sim$ & $\ddot{n}$ & ำ & $\grave{N}$ & $\stackrel{\sim}{\sim}$ & নิ & లి & $\bar{m}$ & लै & $m$ & ले & ले & eे & n & $\stackrel{\infty}{\infty}$ & ले & 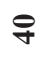 \\
\hline
\end{tabular}


BOSSELAERS J. \& JOCQUÉ R., A new afrotropical genus of Cybaeodinae (Araneae, Liocranidae)

\begin{tabular}{|c|c|c|c|c|c|c|c|c|c|c|c|c|c|c|c|c|c|c|c|c|}
\hline Golo & 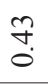 & $\stackrel{n}{0}$ & $\stackrel{n}{\mathfrak{0}}$ & $\stackrel{n}{\mathfrak{0}}$ & 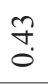 & $\begin{array}{l}8 \\
0 \\
0\end{array}$ & ?ִ & $\stackrel{8}{8}$ & 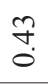 & $\underset{-}{8}$ & $\stackrel{\stackrel{?}{\circ}}{\circ}$ & $\begin{array}{l}\text { ก̊ } \\
\text { ? }\end{array}$ & ?ె? & $\stackrel{n}{\mathfrak{0}}$ & $\begin{array}{l}8 \\
0 \\
0\end{array}$ & m? & $\stackrel{n}{\mathfrak{0}}$ & $\underset{-}{8}$ & $\underset{-}{8}$ & 8 \\
\hline ri & ণิ & $\tilde{b}$ & $\hat{\sigma}$ & $\tilde{b}$ & $\stackrel{8}{:}$ & $\stackrel{8}{\circ}$ & $\stackrel{n}{\stackrel{0}{0}}$ & $\stackrel{8}{8}$ & $\stackrel{8}{:}$ & $\stackrel{8}{8}$ & $\begin{array}{l}0 \\
n \\
0\end{array}$ & $\stackrel{n}{\circ}$ & in & $\stackrel{8}{\circ}$ & $\begin{array}{l}\text { Oे } \\
\stackrel{0}{0}\end{array}$ & $\begin{array}{l}\text { ? } \\
0\end{array}$ & ๙ু & $\stackrel{8}{\circ}$ & 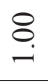 & 8 \\
\hline ci & $\stackrel{m}{m}$ & $\stackrel{n}{n}$ & \begin{tabular}{l}
$\stackrel{n}{n}$ \\
\hdashline
\end{tabular} & $\stackrel{?}{?}$ & ঙ̊ & $\stackrel{m}{m}$ & $\stackrel{\sim}{a}$ & $\stackrel{8}{8}$ & ֻ̊ & $\stackrel{8}{8}$ & $\stackrel{m}{?}$ & $\stackrel{2}{\tilde{c}}$ & $\stackrel{2}{\tilde{c}}$ & $\begin{array}{l}\hat{\sigma} \\
\dot{0}\end{array}$ & $\stackrel{m}{m}$ & $\stackrel{2}{\tilde{c}}$ & $\stackrel{?}{?}$ & $\stackrel{8}{8}$ & $\stackrel{8}{8}$ & $\stackrel{8}{8}$ \\
\hline Steps & $r$ & $\nabla$ & $\nabla$ & $\nabla$ & $=$ & n & 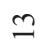 & N & $=$ & $r$ & $=$ & $\underline{2}$ & $\stackrel{0}{-}$ & n & $\simeq$ & $\Xi$ & 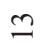 & N & $n$ & $\mathrm{~N}$ \\
\hline Toxoniella taitensis & - & - & - & 0 & - & - & 0 & 0 & - & - & - & 一 & v & 0 & 0 & - & - & - & 0 & - \\
\hline Toxoniella rogoae & - & - & - & 0 & - & - & 0 & 0 & - & - & - & - & $N$ & 0 & 0 & - & - & - & 0 & 一 \\
\hline Scotina palliardii & 0 & 0 & 0 & - & 0 & ๙. & - & 0 & 0 & 0 & $\sim$ & 0 & 0 & 0 & - & 0 & 0 & - & - & - \\
\hline Scotina gracilipes & 0 & 0 & 0 & - & 0 & r. & - & 0 & 0 & 0 & v & o & ○ & 0 & - & 0 & 0 & - & - & - \\
\hline Scotina celans & 0 & 0 & 0 & - & $\circ$ & a. & - & 0 & $\circ$ & 0 & v & ○ & ○ & 0 & - & ○ & 0 & - & - & - \\
\hline Sagana rutilans & 0 & 0 & 0 & - & - & - & - & 0 & - & - & - & - & $\sim$ & 0 & 0 & $\sim$ & - & - & 0 & - \\
\hline Neoanagraphis chamberlini & 0 & 0 & 0 & - & - & - & - & 0 & - & - & 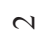 & o & - & - & - & - & 0 & - & 0 & - \\
\hline Mesiotelus tenuissimus & 0 & 0 & 0 & - & 0 & a. & 0 & 0 & - & - & - & - & - & 0 & 0 & - & - & - & 0 & - \\
\hline Mesiotelus cyprius & 0 & 0 & 0 & - & 0 & a. & 0 & 0 & - & - & - & 一 & - & 0 & 0 & - & - & - & 0 & - \\
\hline Liocranum rupicola & 0 & 0 & 0 & - & - & o & o & o & - & - & - & - & - & 0 & 0 & - & - & - & 0 & - \\
\hline Liocranum giersbergi & 0 & 0 & 0 & - & - & - & 0 & 0 & - & - & - & - & $\mathrm{N}$ & 0 & 0 & N & - & - & 0 & - \\
\hline Liocranoeca striata & 0 & 0 & 0 & - & - & 0 & - & 0 & 0 & - & $\sim$ & 0 & 0 & 0 & - & - & 0 & - & 0 & - \\
\hline Hesperocranum rothi & - & 0 & 0 & - & 0 & a. & 0 & 0 & 0 & - & 0 & o & 一 & 0 & 0 & $\neg$ & - & - & 0 & - \\
\hline Cybaeodes marinae & $c$ & 0 & 0 & - & - & - & 0 & 0 & - & - & N & - & $\sim$ & 0 & - & o & - & - & 0 & 一 \\
\hline Cybaeodes mallorcensis & - & 0 & 0 & - & - & - & 0 & 0 & - & - & $\sim$ & 一 & $\sim$ & 0 & 0 & 0 & - & - & 0 & - \\
\hline Cteniogaster toxarchus & 0 & 0 & 0 & - & - & - & 0 & 0 & 0 & - & - & - & v & 0 & 0 & - & - & - & 0 & 一 \\
\hline Cteniogaster hexomma & 0 & 0 & 0 & - & - & - & ○ & $\circ$ & 0 & - & N & - & a. & 0 & a. & a. & - & a. & a. & 一 \\
\hline Cteniogaster conviva & 0 & 0 & 0 & - & - & - & 0 & 0 & 0 & - & $\sim$ & 一 & 一 & 0 & 0 & $\sim$ & - & - & 0 & - \\
\hline Apostenus humilis & 0 & 0 & 0 & - & 0 & ๙. & - & 0 & 0 & 0 & - & 0 & $N$ & 0 & - & 0 & 0 & - & $\sim$ & - \\
\hline Apostenus fuscus & 0 & 0 & 0 & - & 0 & a. & - & 0 & 0 & 0 & N & o & $\mathrm{N}$ & 0 & - & 0 & 0 & - & N & - \\
\hline Agroeca proxima & - & 0 & 0 & - & - & - & - & 0 & - & - & v & o & o & 0 & - & - & 0 & - & 0 & 一 \\
\hline Agroeca parva & 0 & 0 & 0 & - & 0 & ๙. & - & 0 & - & - & $\sim$ & 0 & 0 & 0 & - & 0 & 0 & - & 0 & - \\
\hline Agroeca cuprea & 0 & 0 & 0 & - & 0 & a. & - & 0 & - & - & N & o & 0 & 0 & - & 0 & 0 & - & 0 & - \\
\hline Agroeca brunnea & 0 & 0 & 0 & - & - & - & - & 0 & - & - & $\sim$ & 0 & 0 & 0 & - & - & 0 & - & 0 & 一 \\
\hline Agraecina lineata & 0 & 0 & 0 & - & 0 & a. & 0 & 0 & - & - & 0 & 一 & o & 0 & - & - & 0 & - & 0 & - \\
\hline Creugas bajulus & - & - & - & 0 & - & 0 & - & - & - & N & $\sim$ & 0 & - & - & 0 & $N$ & 0 & 0 & 0 & 0 \\
\hline Corinna nitens & 0 & - & - & 0 & - & - & - & - & 0 & v & v & o & o & v & - & v & 0 & 0 & 0 & 0 \\
\hline Characters & $F$ & $\mathcal{F}$ & $\mathscr{F}$ & $\exists$ & 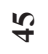 & $\stackrel{f}{t}$ & F & $\stackrel{\infty}{+}$ & $\vec{q}$ & in & $\bar{n}$ & กี & $i n$ & 品 & in & in & in & in & की & 8 \\
\hline
\end{tabular}




\begin{tabular}{|c|c|c|c|c|c|c|c|c|c|c|c|c|c|c|c|c|c|c|c|c|}
\hline Golo & $\begin{array}{l}8 \\
0 \\
0\end{array}$ & $\underset{-}{8}$ & $\begin{array}{l}8 \\
0 \\
0\end{array}$ & $\underset{-}{8}$ & $\stackrel{n}{\stackrel{2}{0}}$ & $\begin{array}{l}\text { ?ִ } \\
0\end{array}$ & $\stackrel{n}{a}$ & $\begin{array}{l}8 \\
0\end{array}$ & $\stackrel{n}{\stackrel{2}{0}}$ & $\underset{-}{8}$ & $\stackrel{n}{\stackrel{2}{0}}$ & $\stackrel{n}{\mathfrak{0}}$ & $\begin{array}{l}8 \\
\stackrel{0}{0}\end{array}$ & $\begin{array}{l}8 \\
0 \\
0\end{array}$ & $\begin{array}{l}8 \\
0 \\
0\end{array}$ & $\underset{-}{8}$ & $\underset{-}{8}$ & $\underset{-}{8}$ & $\underset{-}{8}$ & 8 \\
\hline ri & $\stackrel{\infty}{\stackrel{\infty}{0}}$ & $\underset{-}{8}$ & $\stackrel{8}{:}$ & 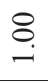 & $\begin{array}{l}\infty \\
\stackrel{0}{0}\end{array}$ & $\begin{array}{l}\text { ? } \\
0\end{array}$ & $\stackrel{8}{\circ}$ & $\hat{\sigma}$ & ๙ૂ & 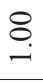 & $\begin{array}{l}\infty \\
\infty \\
0\end{array}$ & $\begin{array}{l}0 \\
\infty \\
0\end{array}$ & $\vec{i}$ & $\begin{array}{l}\text { Oे } \\
\stackrel{0}{0}\end{array}$ & $\begin{array}{l}\text { OL } \\
\stackrel{0}{0}\end{array}$ & $\stackrel{8}{\circ}$ & $\stackrel{8}{\circ}$ & $\stackrel{8}{\circ}$ & $\stackrel{8}{\circ}$ & $\stackrel{8}{-}$ \\
\hline ci & $\stackrel{m}{0}$ & $\stackrel{8}{\circ}$ & $\stackrel{m}{m}$ & $\stackrel{8}{\circ}$ & ?ִ & $\stackrel{\overbrace{}}{\tilde{c}}$ & $\begin{array}{l}\text { ?ִ } \\
\text { ñ }\end{array}$ & $\stackrel{m}{\stackrel{m}{0}}$ & $\begin{array}{l}\text { ?ִ } \\
\text { ?ִ }\end{array}$ & $\stackrel{8}{\circ}$ & $\stackrel{1}{6}$ & $\stackrel{n}{\mathfrak{0}}$ & $\stackrel{m}{m}$ & $\stackrel{m}{\tilde{c}}$ & $\stackrel{m}{m}$ & $\stackrel{8}{\circ}$ & $\stackrel{-}{\circ}$ & $\stackrel{-}{\circ}$ & $\stackrel{8}{\circ}$ & $\stackrel{8}{\circ}$ \\
\hline Steps & $\stackrel{ }{-}$ & $N$ & 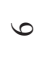 & $m$ & 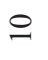 & $r$ & $\sim$ & $r$ & $\stackrel{m}{-}$ & $\sim$ & $\stackrel{0}{-}$ & $\stackrel{0}{-}$ & $\infty$ & $\simeq$ & $\simeq$ & $\sim$ & $a$ & 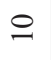 & N & in \\
\hline Toxoniella taitensis & - & 0 & 0 & ๙. & - & 0 & 0 & 0 & - & 0 & - & n & 0 & - & 0 & ๙. & 0 & 0 & 0 & 一 \\
\hline Toxoniella rogoae & - & 0 & 0 & a. & - & 0 & 0 & ○ & - & 0 & - & n & 0 & - & 0 & a. & 0 & 0 & 0 & 一 \\
\hline Scotina palliardii & 0 & 0 & 0 & a. & 0 & 0 & 0 & - & 0 & 0 & N & - & - & 0 & - & 0 & - & - & 0 & 0 \\
\hline Scotina gracilipes & 0 & 0 & 0 & a. & 0 & 0 & 0 & - & 0 & 0 & v & - & - & 0 & - & 0 & - & - & 0 & 0 \\
\hline Scotina celans & 0 & 0 & 0 & a. & 0 & 0 & 0 & - & 0 & 0 & v & - & - & 0 & - & $\circ$ & - & - & 0 & 0 \\
\hline Sagana rutilans & 0 & 0 & $\circ$ & a. & $\circ$ & 0 & 0 & $\longrightarrow$ & 0 & $\circ$ & - & $\sim$ & - & - & ○ & $\sigma$. & 0 & 0 & $\circ$ & - \\
\hline Neoanagraphis chamberlini & o & o & 0 & ๙. & - & - & o & - & - & o & $\mathrm{r}$ & - & - & - & 0 & r. & 0 & 0 & 0 & - \\
\hline Mesiotelus tenuissimus & 0 & 0 & 0 & $\sigma$. & 0 & 0 & 0 & 0 & 0 & 0 & 0 & 0 & 0 & - & 0 & $\sigma$. & 0 & 0 & 0 & - \\
\hline Mesiotelus cyprius & 0 & 0 & 0 & a. & 0 & 0 & 0 & 0 & 0 & 0 & 0 & 0 & 0 & - & 0 & $\sigma$. & $\circ$ & 0 & 0 & 一 \\
\hline Liocranum rupicola & o & o & 0 & r. & 0 & o & o & ○ & o & o & 0 & 0 & 0 & - & o & r. & 0 & 0 & 0 & - \\
\hline Liocranum giersbergi & 0 & 0 & 0 & a. & 0 & 0 & 0 & 0 & $\circ$ & 0 & 0 & 0 & 0 & - & 0 & $\sigma$. & $\circ$ & 0 & 0 & - \\
\hline Liocranoeca striata & 0 & 0 & 0 & a. & - & - & - & - & - & 0 & $\sim$ & - & - & 0 & - & 0 & $\circ$ & $\circ$ & 0 & - \\
\hline Hesperocranum rothi & 0 & 0 & 0 & a. & 0 & 0 & 0 & - & 0 & 0 & - & v & 0 & 0 & 0 & a. & 0 & 0 & 0 & - \\
\hline Cybaeodes marinae & - & 0 & 0 & a. & - & - & 0 & 一 & - & $\circ$ & - & $m$ & - & - & - & - & 0 & 0 & 0 & - \\
\hline Cybaeodes mallorcensis & - & 0 & 0 & ๙. & - & - & 0 & - & - & 0 & - & $m$ & - & - & - & - & 0 & 0 & 0 & - \\
\hline Cteniogaster toxarchus & $\neg$ & 0 & - & - & - & - & 0 & - & - & 0 & v & - & - & 0 & 0 & a. & 0 & 0 & 0 & - \\
\hline Cteniogaster hexomma & - & 0 & - & - & - & - & 0 & 一 & - & 0 & $\sim$ & - & - & 0 & 0 & $\sigma$. & 0 & 0 & 0 & - \\
\hline Cteniogaster conviva & - & 0 & - & - & - & 0 & 0 & - & - & 0 & $\sim$ & - & - & 0 & 0 & $\sigma$. & 0 & 0 & 0 & - \\
\hline Apostenus humilis & o & o & 0 & $\sigma$. & 0 & 0 & 0 & - & 0 & 0 & N & - & - & 0 & - & 0 & - & - & 0 & 0 \\
\hline Apostenus fuscus & - & 0 & - & 0 & 0 & 0 & 0 & - & 0 & 0 & $\sim$ & - & - & 0 & - & 0 & - & - & 0 & 0 \\
\hline Agroeca proxima & 0 & 0 & 0 & a. & 0 & 0 & 0 & - & - & 0 & $\sim$ & - & - & 0 & - & 0 & - & - & ○ & - \\
\hline Agroeca parva & 0 & o & 0 & $\sigma$. & 0 & 0 & 0 & - & - & 0 & N & - & - & 0 & - & 0 & - & - & 0 & - \\
\hline Agroeca cuprea & 0 & 0 & - & 0 & 0 & 0 & 0 & - & - & 0 & $\sim$ & - & - & 0 & - & 0 & - & - & 0 & - \\
\hline Agroeca brunnea & 0 & 0 & - & 0 & 0 & 0 & 0 & $\longrightarrow$ & - & 0 & $\sim$ & - & - & 0 & - & 0 & - & - & 0 & - \\
\hline Agraecina lineata & 0 & o & 0 & $\sigma$. & - & - & 0 & - & - & 0 & N & - & - & 0 & 0 & $\sigma$. & 0 & - & 0 & - \\
\hline Creugas bajulus & - & - & 0 & o. & 0 & 0 & 0 & - & 0 & - & $N$ & - & - & - & 0 & $\sigma$. & 0 & 0 & - & - \\
\hline Corinna nitens & 一 & 一 & 0 & $\sigma$. & 0 & 0 & - & 0 & 0 & - & N & - & 0 & - & 0 & $\sigma$. & 0 & 0 & - & - \\
\hline Characters & 5 & $\widetilde{\sigma}$ & 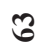 & $\vec{b}$ & 8 & 8 & 5 & $\infty$ & 8 & R & $F$ & $n$ & $m$ & $\pi$ & 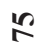 & 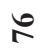 & N & $\stackrel{\infty}{\infty}$ & 2 & $\infty$ \\
\hline
\end{tabular}


BOSSELAERS J. \& JOCQUÉ R., A new afrotropical genus of Cybaeodinae (Araneae, Liocranidae)

\begin{tabular}{|c|c|c|c|c|c|c|c|c|c|c|c|c|c|c|c|c|c|c|c|}
\hline Goloboff fit & $\begin{array}{l}8 \\
0 \\
0\end{array}$ & $\stackrel{n}{0}$ & 8 & $\stackrel{n}{0}$ & ?̊n & $\stackrel{\infty}{\infty}$ & $\stackrel{?}{+}$ & $\begin{array}{l}8 \\
0 \\
0\end{array}$ & $\underset{-}{8}$ & 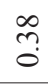 & $\stackrel{8}{-}$ & 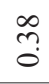 & $\frac{n}{0}$ & $\begin{array}{l}8 \\
0 \\
0\end{array}$ & ?̊n & $\stackrel{n}{0}$ & $\stackrel{8}{-}$ & $\stackrel{8}{-}$ & $\stackrel{n}{0}$ \\
\hline ri & $\stackrel{m}{0}$ & ?̊ & $\stackrel{8}{\circ}$ & $\frac{n}{2}$ & $\hat{n}$ & $\stackrel{n}{n}$ & $\begin{array}{l}\text { ț } \\
\text { o. }\end{array}$ & $\stackrel{\infty}{\infty}$ & $\stackrel{8}{\circ}$ & $\stackrel{+}{+}$ & $\stackrel{8}{8}$ & in & $\stackrel{8}{\circ}$ & $\begin{array}{l}\mathscr{\infty} \\
0 \\
0\end{array}$ & ?̊ & ?̊ & $\stackrel{8}{\circ}$ & $\stackrel{8}{\circ}$ & $\begin{array}{l}\infty \\
\infty \\
0\end{array}$ \\
\hline ci & $\stackrel{m}{?}$ & ?̊n & $\stackrel{8}{\circ}$ & ?̊n & $\stackrel{2}{n}$ & $\frac{1}{0}$ & ?̊? & $\stackrel{m}{0}$ & $\stackrel{8}{\circ}$ & $\frac{1}{0}$ & $\stackrel{8}{\circ}$ & $\frac{1}{0}$ & in & $\stackrel{m}{?}$ & $\stackrel{2}{n}$ & ?n & $\stackrel{8}{\circ}$ & $\stackrel{8}{\circ}$ & ?n \\
\hline Steps & $\forall$ & $m$ & $\sim$ & $n$ & $\infty$ & $\simeq$ & $\stackrel{0}{-1}$ & $=$ & $N$ & 으 & 6 & $\simeq$ & $N$ & $\underline{2}$ & $r$ & $m$ & $N$ & $N$ & $a$ \\
\hline Toxoniella taitensis & - & 0 & - & 0 & 0 & 0 & 0 & 0 & 0 & 0 & 0 & - & 0 & - & 0 & 0 & 0 & - & 0 \\
\hline Toxoniella rogoae & - & 0 & - & 0 & 0 & 0 & - & 0 & 0 & 0 & 0 & - & 0 & - & 0 & 0 & 0 & - & 0 \\
\hline Scotina palliardii & $\curvearrowright$. & $\curvearrowright$. & - & 0 & 0 & - & $n$ & 0 & 0 & - & 0 & 0 & 0 & 0 & 0 & 0 & 0 & - & 0 \\
\hline Scotina gracilipes & $\sim$. & $\sigma$. & - & 0 & 0 & - & in & 0 & 0 & - & 0 & 0 & 0 & 0 & 0 & 0 & 0 & - & 0 \\
\hline Scotina celans & a. & ๙. & - & 0 & 0 & - & $n$ & 0 & 0 & - & 0 & 0 & 0 & 0 & 0 & 0 & 0 & - & 0 \\
\hline Sagana rutilans & - & 0 & - & - & - & - & 一 & 一 & 0 & - & - & - & 0 & - & 0 & 0 & 0 & - & - \\
\hline Neoanagraphis chamberlini & - & 0 & - & 0 & 0 & - & $m$ & 0 & 0 & 0 & 0 & - & - & 0 & 0 & - & 0 & - & 0 \\
\hline Mesiotelus tenuissimus & - & 0 & - & 0 & 0 & 0 & 0 & - & 0 & - & - & 0 & 0 & - & 0 & 0 & 0 & - & - \\
\hline Mesiotelus cyprius & - & 0 & - & 0 & 0 & 0 & 0 & - & 0 & - & - & 0 & 0 & - & 0 & 0 & 0 & - & - \\
\hline Liocranum rupicola & - & 0 & - & 0 & 0 & 0 & 0 & - & 0 & - & - & 0 & 0 & - & 0 & 0 & 0 & - & - \\
\hline Liocranum giersbergi & - & 0 & - & 0 & 0 & 0 & 0 & - & 0 & - & - & 0 & 0 & - & 0 & 0 & 0 & - & - \\
\hline Liocranoeca striata & - & 0 & - & 0 & 0 & 0 & - & 0 & 0 & 0 & 0 & 0 & 0 & - & 0 & 0 & 0 & - & 0 \\
\hline Hesperocranum rothi & - & 0 & - & 0 & 0 & - & N & - & 0 & - & - & - & 0 & 0 & 0 & 0 & 0 & - & 0 \\
\hline Cybaeodes marinae & - & 0 & - & 0 & - & - & - & - & 0 & - & 0 & - & 0 & - & 0 & - & 0 & - & - \\
\hline Cybaeodes mallorcensis & - & 0 & - & 0 & - & - & - & 0 & 0 & - & 0 & - & 0 & - & 0 & - & 0 & - & - \\
\hline Cteniogaster toxarchus & - & 0 & - & 0 & 0 & 0 & 0 & 0 & 0 & 0 & 0 & 0 & 0 & - & 0 & 0 & 0 & - & 0 \\
\hline Cteniogaster hexomma & - & 0 & - & 0 & 0 & 0 & 0 & 0 & 0 & 0 & 0 & 0 & 0 & - & 0 & 0 & 0 & - & 0 \\
\hline Cteniogaster conviva & 0 & - & - & 0 & 0 & 0 & 0 & 0 & 0 & 一 & 0 & 一 & 0 & - & 0 & 0 & 0 & - & 0 \\
\hline Apostenus humilis & $\sigma$. & $\sigma$. & - & 0 & - & 0 & 0 & 0 & 0 & - & 0 & 0 & 0 & 0 & - & 0 & 0 & 0 & $\sigma$. \\
\hline Apostenus fuscus & $\sigma$. & c. & - & 0 & - & 0 & 0 & 0 & 0 & - & 0 & - & - & 0 & 0 & 0 & 0 & 0 & r. \\
\hline Agroeca proxima & - & 0 & - & - & 0 & - & $\forall$ & 0 & 0 & 一 & 0 & - & 0 & 0 & - & 0 & 0 & 一 & 0 \\
\hline Agroeca parva & - & o & - & - & 0 & - & 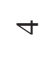 & 0 & 0 & 0 & 0 & - & 0 & 0 & - & 0 & 0 & - & 0 \\
\hline Agroeca cuprea & - & 0 & - & - & 0 & - & $\nabla$ & - & 0 & 0 & 0 & - & 0 & 0 & - & 0 & 0 & - & 0 \\
\hline Agroeca brunnea & - & 0 & - & - & 0 & - & $\forall$ & 一 & 0 & 一 & 0 & - & 0 & 0 & 一 & 0 & 0 & - & 0 \\
\hline Agraecina lineata & 0 & 0 & - & 0 & - & 0 & - & 0 & 0 & - & 0 & 0 & 0 & 0 & - & 0 & 0 & - & 0 \\
\hline Creugas bajulus & 0 & - & 0 & $\sigma$. & - & 0 & 0 & - & - & 0 & 0 & - & 0 & 0 & 一 & 0 & - & - & - \\
\hline Corinna nitens & 0 & - & 0 & $\curvearrowright$ & - & 0 & 一 & 一 & 一 & 0 & 0 & - & 0 & 0 & 0 & 0 & - & - & - \\
\hline Characters & $\infty$ & లే & $\infty$ & ప & $\infty$ & $\infty$ & $\infty$ & $\infty$ & $\infty$ & ฉ & $\bar{a}$ & $\tilde{\sigma}$ & a & at & å & ๙ & à & $\stackrel{\circ}{a}$ & 天े \\
\hline
\end{tabular}

